\title{
A century of exercise physiology: key concepts on coupling respiratory oxygen flow to muscle energy demand during exercise
}

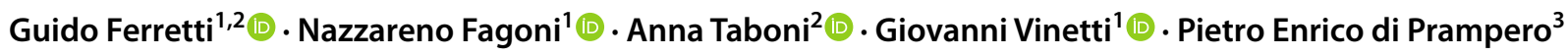

Received: 15 September 2021 / Accepted: 25 January 2022 / Published online: 26 February 2022

(c) The Author(s) 2022

\begin{abstract}
After a short historical account, and a discussion of Hill and Meyerhof's theory of the energetics of muscular exercise, we analyse steady-state rest and exercise as the condition wherein coupling of respiration to metabolism is most perfect. The quantitative relationships show that the homeostatic equilibrium, centred around arterial $\mathrm{pH}$ of 7.4 and arterial carbon dioxide partial pressure of $40 \mathrm{mmHg}$, is attained when the ratio of alveolar ventilation to carbon dioxide flow $\left(\dot{V}_{A} / \dot{V}_{R} C O_{2}\right)$ is -21.6 . Several combinations, exploited during exercise, of pertinent respiratory variables are compatible with this equilibrium, allowing adjustment of oxygen flow to oxygen demand without its alteration. During exercise transients, the balance is broken, but the coupling of respiration to metabolism is preserved when, as during moderate exercise, the respiratory system responds faster than the metabolic pathways. At higher exercise intensities, early blood lactate accumulation suggests that the coupling of respiration to metabolism is transiently broken, to be re-established when, at steady state, blood lactate stabilizes at higher levels than resting. In the severe exercise domain, coupling cannot be re-established, so that anaerobic lactic metabolism also contributes to sustain energy demand, lactate concentration goes up and arterial pH falls continuously. The $\dot{V}_{A} / \dot{V}_{R} C O_{2}$ decreases below -21.6 , because of ensuing hyperventilation, while lactate keeps being accumulated, so that exercise is rapidly interrupted. The most extreme rupture of the homeostatic equilibrium occurs during breath-holding, because oxygen flow from ambient air to mitochondria is interrupted. No coupling at all is possible between respiration and metabolism in this case.
\end{abstract}

Keywords Oxygen flow · Oxygen consumption · Ventilation · Diffusion · Cardiac output · Haemoglobin · Metabolism · Energetics $\cdot$ Exercise transient $\cdot$ Breath-holding

\begin{tabular}{|c|c|c|c|}
\hline \multicolumn{2}{|c|}{ Abbreviations } & \multirow[t]{2}{*}{$b$} & \multirow{2}{*}{$\begin{array}{l}\text { Constant relating rate of lactate accumulation } \\
\text { to rate of ATP resynthesis }\end{array}$} \\
\hline A & Surface area & & \\
\hline$a$ & $\begin{array}{l}\text { Constant relating rate of phosphocreatine } \\
\text { hydrolysis to rate of ATP resynthesis }\end{array}$ & BTPS & $\begin{array}{l}\text { Body temperature and pressure, saturated } \\
\text { with water vapour }\end{array}$ \\
\hline ADP & Adenosine-di-phosphate & $c$ & Constant relating rate of oxygen consumption \\
\hline ATP & Adenosine-tri-phosphate & & to rate of ATP resynthesis \\
\hline$\stackrel{\leftrightarrow \dot{T T P}}{\longrightarrow \dot{T} D}$ & Rate of ATP hydrolysis & $\mathrm{C}_{a} \mathrm{CO}_{2}$ & Carbon dioxide concentration in arterial \\
\hline$A \dot{T} P$ & Rate of ATP resynthesis & & blood \\
\hline & & $\mathrm{C}_{a} \mathrm{O}_{2}$ & Oxygen concentration in arterial blood \\
\hline \multicolumn{2}{|c|}{ Communicated by Michael Lindinger. } & & $\begin{array}{l}\text { between the STPD-to-BTPS and the } P_{A} \mathrm{CO}_{2} \\
\text {-to- } F_{A} \mathrm{CO}_{2} \text { conversion factors }\end{array}$ \\
\hline \multirow{2}{*}{\multicolumn{2}{|c|}{$\begin{array}{l}\triangle \text { Guido Ferretti } \\
\text { guido.ferretti@unibs.it }\end{array}$}} & $\mathrm{CO}$ & Carbon monoxide \\
\hline & & $\mathrm{CO}_{2}$ & Carbon dioxide \\
\hline \multicolumn{2}{|c|}{$\begin{array}{l}\text { Dipartimento di Medicina Molecolare e Traslazionale, } \\
\text { Università di Brescia, Brescia, Italy }\end{array}$} & $\mathrm{C}_{\bar{\nu}} \mathrm{CO}_{2}$ & $\begin{array}{l}\text { Carbon dioxide concentration in mixed } \\
\text { venous blood }\end{array}$ \\
\hline \multicolumn{2}{|c|}{$\begin{array}{l}\text { Département d'Anesthésiologie, Pharmacologie et Soins } \\
\text { Intensifs, Université de Genève, Genève, Switzerland }\end{array}$} & $\begin{array}{l}\mathrm{C}_{\bar{v}} \mathrm{O}_{2} \\
d\end{array}$ & $\begin{array}{l}\text { Oxygen concentration in mixed venous blood } \\
\text { Diffusion constant }\end{array}$ \\
\hline \multicolumn{2}{|c|}{$\begin{array}{l}\text { Professore Emerito di Fisiologia, Università di Udine, Udine, } \\
\text { Italy }\end{array}$} & $D_{L}$ & $\begin{array}{l}\text { Lung diffusion capacity (conductance of the } \\
\text { alveolar-capillary barrier) }\end{array}$ \\
\hline
\end{tabular}




\begin{tabular}{|c|c|c|c|}
\hline $\begin{array}{l}d \dot{M} \\
D O_{2 A L}\end{array}$ & $\begin{array}{l}\text { Infinitesimal molar flow of a gas } \\
\text { Alactic oxygen deficit }\end{array}$ & $P_{\bar{v}} \mathrm{O}_{2}$ & $\begin{array}{l}\text { Oxygen partial pressure in mixed venous } \\
\text { blood }\end{array}$ \\
\hline \multirow[t]{2}{*}{$D O_{2 L A}$} & \multirow{2}{*}{$\begin{array}{l}\text { Fraction of the oxygen deficit represented by } \\
\text { early lactate }\end{array}$} & $\dot{Q}$ & Total blood flow, or cardiac output \\
\hline & & $\dot{Q}_{a} \mathrm{O}_{2}$ & Oxygen flow in arterial blood, or oxygen \\
\hline$D O_{2 M}$ & \multicolumn{2}{|l|}{ Muscular oxygen deficit } & delivery \\
\hline$E$ & Energy & $q_{c}$ & Lung capillary blood volume \\
\hline$\dot{E}$ & Overall metabolic power & $Q_{s}$ & Stroke volume of the heart \\
\hline \multirow{2}{*}{$\mathrm{F}_{\mathrm{A}} \mathrm{CO}_{2}$} & \multirow{2}{*}{$\begin{array}{l}\text { Carbon dioxide fraction in alveolar air } \\
\text { Carbon dioxide fraction in expired air }\end{array}$} & $\dot{Q}_{\bar{v}} \mathrm{O}_{2}$ & Oxygen flow in mixed venous blood, or oxy- \\
\hline & & & gen return \\
\hline $\mathrm{F}_{E} \mathrm{O}_{2}$ & Oxygen fraction in expired air & $R$ & Energy introduced into $1 \mathrm{~mol}$ of gas by a unit \\
\hline$f_{H}$ & Heart rate & & increase in temperature \\
\hline $\mathrm{F}_{\mathrm{I}} \mathrm{CO}_{2}$ & Carbon dioxide fraction in inspired air & $r$ & Rest (suffix) \\
\hline$F_{I} N_{2}$ & Nitrogen fraction in inspired air & $R_{p}$ & Total peripheral resistance \\
\hline $\mathrm{F}_{\mathrm{I}} \mathrm{O}_{2}$ & Oxygen fraction in inspired air & $R Q_{B}$ & Blood respiratory quotient \\
\hline$[\mathrm{Hb}]$ & Blood haemoglobin concentration & $R Q_{L}$ & Lung respiratory quotient \\
\hline$K_{e}$ & Equilibration coefficient & $R Q_{M}$ & Metabolic respiratory quotient \\
\hline$L$ & Length, thickness & $R R i$ & $\mathrm{R}-\mathrm{R}$ interval, or time between two consecu- \\
\hline$\dot{L a}$ & \multicolumn{2}{|l|}{ Rate of lactate accumulation in blood } & tive $\mathrm{R}$ peaks on an electrocardiogram \\
\hline \multirow[t]{2}{*}[La]{$_{e}$} & Early lactate, i.e., net blood lactate accumula- & $s$ & Solubility constant \\
\hline & tion during the exercise transient & $\mathrm{S}_{a} \mathrm{O}_{2}$ & Arterial oxygen saturation \\
\hline \multirow[t]{2}{*}{$n$} & \multirow{2}{*}{$\begin{array}{l}\text { Number of moles (of gas in a volume or of } \\
\text { oxygen bound to a mole of haemoglobin) }\end{array}$} & ss & Steady state (suffix) \\
\hline & & STPD & Standard temperature and pressure, dry \\
\hline $\mathrm{O}_{2}$ & Oxygen & $T$ & Temperature \\
\hline$P$ & Pressure & $t$ & Time \\
\hline $\bar{P}$ & Mean arterial pressure & $T_{0}$ & Temperature at which $E=0 \mathrm{~J}$ \\
\hline \multirow[t]{2}{*}{$P_{50}$} & \multirow{2}{*}{$\begin{array}{l}\text { Oxygen partial pressure sustaining a satura- } \\
\text { tion of } 0.5\end{array}$} & $t^{1 / 2}$ & Half-time \\
\hline & & $t_{c}$ & Contact time \\
\hline \multirow[t]{2}{*}{$\mathrm{P}_{A} \mathrm{CO}_{2}$} & \multirow{2}{*}{$\begin{array}{l}\text { Partial pressure of carbon dioxide in alveolar } \\
\text { air }\end{array}$} & $V$ & Volume \\
\hline & & $\dot{V}$ & Gas flow \\
\hline \multirow[t]{2}{*}{$\mathrm{P}_{a} \mathrm{CO}_{2}$} & \multirow{2}{*}{$\begin{array}{l}\text { Partial pressure of carbon dioxide in arterial } \\
\text { blood }\end{array}$} & $\dot{V}_{A}$ & Alveolar ventilation \\
\hline & & $\dot{V}_{A} / \dot{Q}$ & Ventilation/perfusion ratio \\
\hline$P_{A} O_{2}$ & Oxygen partial pressures in alveolar air & $\dot{V}_{A} / \dot{V}_{R} \mathrm{CO}_{2}$ & Ratio between alveolar ventilation and lung \\
\hline $\mathrm{P}_{a} \mathrm{O}_{2}$ & Oxygen partial pressure in arterial blood & & carbon dioxide flow \\
\hline$P_{B}$ & Barometric pressure & $\dot{\mathrm{V} C O} \mathrm{O}_{2}$ & Rate of carbon dioxide production \\
\hline $\mathrm{P}_{c} \mathrm{O}_{2}$ & Oxygen partial pressure in lung capillaries & $\dot{V}_{E}$ & Total expired air flow, or expiratory \\
\hline \multirow{2}{*}{$P_{c^{\prime}} O_{2}$} & \multirow{2}{*}{$\begin{array}{l}\text { Oxygen partial pressure at the end of lung } \\
\text { capillaries }\end{array}$} & & ventilation \\
\hline & & $\dot{V}_{I}$ & Total inspired air flow, or inspiratory \\
\hline $\mathrm{PCr}$ & Phosphocreatine & & ventilation \\
\hline$P \dot{C} r$ & $\begin{array}{l}\text { Rate of phosphocreatine decrease in contract- } \\
\text { ing muscles }\end{array}$ & $V_{L} O_{2}$ & $\begin{array}{l}\text { Volume of oxygen crossing the alveolar-cap- } \\
\text { illary barrier during a constant-load exercise }\end{array}$ \\
\hline$P_{c}(\delta)$ & $\begin{array}{l}\text { Capillary pressure at distance } \delta \text { from the } \\
\text { venous entrance }\end{array}$ & $\dot{V}_{L} O_{2}$ & $\begin{array}{l}\text { Diffusive oxygen flow across the alveolar- } \\
\text { capillary membrane }\end{array}$ \\
\hline PFK & Phospho-fructo-kinase & $\dot{V} O_{2}$ & Rate of oxygen consumption \\
\hline $\mathrm{pH}$ & $\begin{array}{l}\text { Negative logarithm of hydrogen ion } \\
\text { concentration }\end{array}$ & $V O_{2 M}$ & $\begin{array}{l}\text { Volume of oxygen extracted by the contract- } \\
\text { ing muscles }\end{array}$ \\
\hline $\mathrm{Pi}$ & Inorganic phosphate & $\dot{V} O_{2}^{s}$ & $\dot{\mathrm{V}} \mathrm{O}_{2}$ at steady state \\
\hline $\mathrm{P}_{\mathrm{I}} \mathrm{O}_{2}$ & Oxygen partial pressure in inspired air & $\dot{V} O_{2}^{t}$ & $\dot{V} O_{2}$ at time $t$ \\
\hline$P / L a$ & $\begin{array}{l}\text { Moles of ATP resynthesized per mole of } \\
\text { lactate accumulated }\end{array}$ & $\dot{\mathrm{V}}_{\mathrm{R}} \mathrm{C} \mathrm{O}_{2}$ & $\begin{array}{l}\text { Carbon dioxide flow along the respiratory } \\
\text { system }\end{array}$ \\
\hline P-NMR & Phosphorus-nuclear magnetic resonance & $\dot{V}_{R} O_{2}$ & Oxygen flow along the respiratory system \\
\hline $\mathrm{PO}_{2}$ & Oxygen partial pressure, generic & $V_{v}$ & Venous blood volume \\
\hline $\mathrm{P} / \mathrm{O}_{2}$ & Moles of ATP resynthesized per mole of & $W$ & Work \\
\hline & oxygen consumed & $\Delta G$ & Free energy \\
\hline
\end{tabular}


$\Delta[L a]_{b} \quad$ Net blood lactate accumulation

$\Delta P \quad$ Pressure difference

$\Delta V_{v} O_{2} \quad$ Changes in venous blood oxygen stores

\section{List of symbols}

$\beta$

$\beta c$

$\frac{\beta o}{\beta o}$

$\delta$

$\lambda$

$\tau$

$\tau_{0}$
Blood transport coefficient of a gas, generic Blood transport coefficient of carbon dioxide Blood transport coefficient of oxygen Mean slope of the oxygen equilibrium curve Distance from the venous entrance of a lung capillary

Time constant increase per unit increase of lactate in blood during the exercise transient Oxygen-binding capacity of haemoglobin Time constant Time constant in absence of early lactate accumulation

\section{Introduction}

The celebration of a century of exercise physiology, since the Nobel Prize award to Archibald Vivian Hill (1886-1977) and Otto Fritz Meyerhof (1884-1951) in 1922, is a very ambitious enterprise. In affording it, one has to look back at the impressive developments that took place since then. A comparison of what exercise physiology was in Hill and Meyerhof's days with what it has become nowadays makes people suddenly aware of the astonishingly huge size of the scientific developments occurring in that time lapse. All of a sudden, one realises that, apart from classical exercise physiology, the number of brand new fields of research related to exercise is great indeed, from muscle and exercise molecular biology to biomechanics, from training physiology, to exercise neuroscience and exercise pathophysiology. The modern term exercise science, unknown in Hill and Meyerhof's times, encompasses a wide spectrum of scientific activities related to exercise.

The development of exercise physiology proceeded in parallel with a tremendous societal change. In 1922, most of leisure or sport exercise practise was a privilege of a small number of rich men from aristocracy or high bourgeoisie. The romantic ideas that led to the revival of the Olympic Games inspired them. In 2022, sport has become a widespread popular practice, supporting professional activities of many people, and generating rich and profitable related industrial activities, producing and distributing sport shoes, sportswear, technical tools, training ergometers, ergogenic products, therapeutic tools, and so on. Indeed, the development of exercise physiology, and, more generally speaking, exercise science, proceeded together with, and partly induced, the impressive, enormous societal, industrial, medical, sport evolution that took place, since Hill and Meyerhof established the first basis of "modern" exercise physiology.

We are honoured to contribute to the present celebration of one century of exercise physiology by taking the heavy task of this review on our shoulders. The aim is to discuss and revise from a contemporary holistic perspective, but in the light of historical developments, a very complex concept indeed, that of coupling energy demand by the exercising muscles to energy delivery by the respiratory system. The latter is defined in its broadest sense, as the entire pathway from ambient air to mitochondria. Gas flow along this pathway is sustained by lung ventilation, lung diffusion and perfusion, cardiovascular blood flow and gas transport, peripheral diffusion and perfusion. The functional state of the respiratory system is modulated in such a way as to maintain as much as possible an equilibrium ensuring adequate oxygen delivery to support cell metabolism (at exercise mostly muscle fibres).

The concept of steady state is strictly related to the concept of coupling. Steady state, another intellectual creation of the 1920s' physiology, implies that, whenever the rate of energy metabolism is kept invariant at the level that is necessary to support ATP resynthesis and ATPase activities, whether at rest or at exercise, also the oxygen and carbon dioxide flows, and the physiological variables that set them along the entire respiratory system, remain stable in time. The concepts of coupling and steady state stemmed from and are part of the wider concept of a stable milieu intérieur, which Claude Bernard (1813-1878) created amidst the nineteenth century (Bernard 1859, 1865). This concept implies that the normal physiological state of a living body is ensured by a strict chemico-physical equilibrium, which is maintained by fine regulatory mechanisms throughout the entire life span. Of course, the quantitative relationships at steady state ensuring respiratory-metabolic coupling must respect this equilibrium.

The structure of this review will thus be as follows. After a short account of the main findings up to the end of the nineteenth century, we discuss the revolution represented by Hill and Meyerhof's theory of the energetics of muscular exercise and its refutation. Then, we introduce the concept of steady state and we analyse and discuss the quantitative relationships at steady state, which define the laws by which the coupling of respiration and metabolism occur. Finally, a few examples of rupture of these equilibria in unsteady state conditions are analysed, namely the exercise transient, including severe exercise, and breath-holding.

\section{Antiquity to the nineteenth century}

Classical Greek medicine recognized the muscles as the site of movement, but did not connect them to other functions of the body. A major step forward occurred when dissection was permitted in Alexandria. Therefore, in the early third 
century BC, Herophilus ( $\approx 330-250 \mathrm{BC})$ not only recognized the importance of muscles for movement, but also distinguished between motor nerves and sensitive nerves, reported the connection of the former to muscles, proposed a role for motor nerves in muscle contraction, and eventually established what is very likely the first theory of contraction. Galen (129-201 AD) summarized Herophilus' theory as follows: "during contraction the muscles are filled with pneuma, increase in breadth, but diminish in length". Here, skeletal muscles are clearly defined as the organs of voluntary movement; if muscles are filled with pneuma, perhaps through the nerves, which according to Herophilus contain pneuma, they are contracted, id est active, they shorten and generate movement; at the end of movement, they lose pneuma and relax in a passive manner (von Staden 1989).

These ideas remained substantially unchanged until the Renaissance. The great natural philosophers of that era (sixteenth-seventeenth century) viewed muscles pretty much as the Alexandrian School, although they downgraded the metaphysical concept of pneuma as the origin of contraction. René Descartes (1596-1650) replaced it with the "vital spirits", the behaviour of which should be subjected to physical laws.

The cultural climate underwent drastic changes in that time. Focus moved from a metaphysical to a physical vision of nature, which led to the birth of modern experimental science. This was a real cultural revolution, inasmuch as scientific theory was enslaved to experimental validation, or falsification. The revolutionary spirit of those days, equalled only, although on a smaller scale, by the cultural climate in early Alexandria, gave origin to classical Galilean and Newtonian physics, classical chemistry, new mathematical tools - analytical geometry and calculus were then createdtogether with new specific measurement tools. Among these, the invention of the microscope allowed substantial advances also in the field of muscle physiology: Giovanni Alfonso Borelli (1608-1679), William Croone (1633-1684), Anton van Leeuwenhoek (1632-1723), Niels Steensen (1638-1686), and Jan Swammerdam (1637-1680) established by observation and experiments the fibrous structure of muscle, its cross-striation, and demonstrated that muscle contraction may occur without changes in volume (Needham 1971).

In that time, it became clear that air did not consist of a single gas but was a mixture of different gaseous constituents. Michal Sedziwój (1566-1636) had already proposed that one constituent of air may be a "life-giving" substance. In fact, when kalium nitrate was heated, a gas was liberated that could be collected in flasks and used. According to the Dutch engineer Cornelis Drebbel (1566-1625), that gas could sustain up to 12 men in a submarine rowing longer than $1 \mathrm{~h}$ from Westminster to Greenwich down the river Thames (Poole et al. 2015).
That substance may well correspond to the "nitro-aerial" particles of air, which John Mayow (1643-1679) suggested to be used during contraction, with the simultaneous elimination of a body constituent. This was a totally new concept, which, for the first time in history, suggested a relation of muscle contraction to respiration and a yet vague idea of coupling (Mayow 1681). This suggestion is perhaps the first seed that later grew into the current idea of oxygen utilisation in metabolism associated with carbon dioxide elimination, as long as that gas was probably oxygen.

The ideas of Mayow gained a prominent place in the history of physiology, thanks to the subsequent developments in the chemistry of gases, one of the most exciting scientific adventures of seventeenth and eighteenth centuries. The concept of gas, as that of air, preceded the scientific revolution of seventeenth century. In fact, already Anaximenes of Miletus ( $\approx 586-526$ BC) regarded air, which he called pneuma, as the initial substance of the physical world, although he thought air to be a single, pure gas. The modern physics of air started with Robert Boyle (1627-1691), who described the inverse relationship between pressure and volume in a given quantity of gas. Since the product of pressure times volume is an amount of energy, Boyle's relationship indicated that the amount of energy in a given quantity of gas was a constant.

It took more than one century, with the creation of the concept of temperature and the invention of the thermometer and of the temperature scales, to demonstrate that Boyle's constant varied linearly with temperature. At the end of a long path, Emile Clapeyron (1799-1864) defined, in 1834, what is usually known as the equation of state of ideal gases, and expressed it in this form

$E=P V=n R\left(T-T_{0}\right)$,

where $E, P, V$, and $T$ stand for energy, pressure, volume, and temperature (expressed in ${ }^{\circ} \mathrm{C}$ ), $n$ represents the amount of gas, currently expressed in moles, and $R$, which was called the universal constant of ideal gases, indicates the amount of energy introduced into $1 \mathrm{~mol}$ of gas by a unit increase in temperature. In fact, $R$ is the angular coefficient of the linear relationship between the energy in a given gas quantity and the temperature of the same gas, for $n=1$. Constant $T_{0}$ is the intercept on the $x$-axis of the same relationship and corresponds to the temperature at which $E=0 \mathrm{~J}$. It is equal to $-273.14{ }^{\circ} \mathrm{C}$ and was called absolute zero. When William Thomson, first Baron Kelvin (1824-1907) set $T_{0}$ equal to zero-in fact, he shifted the $x$-axis intercept of the $E$ versus $T$ relationship rightward to have it coinciding with the origin of the axes-, he created the absolute temperature scale, named in his honour. Therefore, if we express temperature in absolute scale (Kelvin degrees, ${ }^{\circ} \mathrm{K}$ ), Eq. (1a) becomes 
$E=P V=n R T$,

thereby setting a relation of direct proportionality between $E$ and $T$. Equation (1b) had numerous remarkable consequences, among which we underline (i) the definition of heat as a form of energy, (ii) the opening of the path that led to the creation of the new concept of entropy, and, in the present niche context, (iii) the formulation of theories of the energetics of muscular exercise. Concerning respiration, Eq. (1b) defined the criteria of quantitative standardization for gas volumes and flows, which were established by the American Physiological Society (Pappenheimer 1950). The conventional expression of air volume and flow in BTPS (body temperature and pressure, saturated with water vapour) and of single gas volume and flow in STPD (standard temperature and pressure, dry) dates back to that time.

A fundamental step forward, providing a chemical basis to the aforementioned assertion of John Mayow, occurred in the second half of the eighteenth century. Joseph Black (1728-1799) discovered carbon dioxide in 1764, which he called "fixed air", and which he reported to be exhaled during respiration. Joseph Priestley (1733-1804) and Carl Wilhelm Scheele (1742-1786) discovered oxygen independently in the 1770s. Priestley, who first published his experiment in 1774 , called it "dephlogisticated air", which means air without phlogiston, which was then thought to be the (metaphysical) fire-like element liberated during combustion processes. In fact, the theory of phlogiston was deeply rooted in eighteenth century chemistry. Georg Ernst Stahl (1660-1734) was a strong advocate of the phlogiston theory. According to him, inflammation (combustion) liberated phlogiston, so that the burned substance was transformed into ashes. Shortly after the identification of oxygen and carbon dioxide, Antoine-Laurent Lavoisier (1743-1794) refuted the phlogiston theory, demonstrating that combustion implies the combination of a fuel with oxygen (theory of oxidation). He also realised that animals, and inter eos humans, consume Priestley's gas, which he called oxygen, and demonstrated that the rate of oxygen consumption $\left(\dot{V} \mathrm{O}_{2}\right)$ increased when a man exercised. Moreover, together with Armand Séguin (1767-1835), he showed that exercise was associated also with an increased elimination of carbon dioxide and an increased production of heat (Séguin and Lavoisier 1789). Combination of these observations led to the notion that the production of mechanical energy in muscle contraction is a chemical process of combustion, in which oxygen reacts with a fuel, yielding carbon dioxide as the end-product, and heat is generated in the process. This meant that exercise was possible thanks to chemical energy transformations in mechanical work and heat, although heat was not yet clearly seen as a form of energy. This was a revolutionary concept indeed, so fraught with consequences for future physiological developments, that we can easily recognize it as the seminal starting point of exercise physiology, without which Hill and Meyerhof's theories, which we celebrate, would not have been possible. In May 1794, during the most radical period of the French Revolution, Lavoisier was condemned to death, allegedly for his previous support to the aristocratic regime, and the guillotine put an end, together with his life, to his revolutionary scientific work.

A necessary consequence of the work by Séguin and Lavoisier was that the process of chemical energy transformation requires oxidation of an organic fuel, which was then unknown. Moreover, a tight match between oxygen consumption and fuel oxidation must be in place, as well as between respiration and muscle energy transformations, since oxygen must be taken from ambient air. Lavoisier believed that combustion occurred in the lungs, and that the generated heat was removed by blood circulation. Adair Crawford (1748-1795) proposed a different theory of animal heat, which we summarize as follows: the oxygen contained in ambient air is converted into carbon dioxide in the lungs, thereby liberating heat; this heat, however, does not increase lung temperature, because of differences in the specific heat of arterial and venous blood. As a consequence, the blood in the pulmonary vein undergoes an increase in specific heat due to heat delivery from the lung. Hence, Crawford resurrected the phlogistic theory, stating that combustibles consisted of ash combined with a fire principle, the "phlogiston", which was liberated during burning. Crawford believed that phlogiston prompted the alleged heat delivery to blood, despite that Lavoisier had already demonstrated the incorrectness of the phlogiston theory. This is a nice example indeed of how difficult it is to dismiss a theory that was proven untrue, when it has become a dogma.

For a more detailed report of the history of classical gas chemistry, we are pleased to direct the readers to the magnificent chapter of the first edition of the Handbook of Physiology, on the history of respiration (Perkins 1964). That chapter discusses, inter alia, the discovery of nitrogen, the definition of the composition of ambient air, the creation of Dalton's law and of the concept of partial pressure of pure gases in a gas mixture, and the formulation of Henry's law, describing the principles that govern the solution of gases in a liquid (plasma, as far as we are concerned). Although all these concepts pertain to the present article, we refrain from discussing them here for reasons of space.

The identification of the fuel supporting the combustion process generating the mechanical energy for organismal movement progressed from the chemical experiments on aliments of the first half of the nineteenth century. Justus von Liebig (1803-1873) and Michel Eugène Chevreul (1786-1889) described carbohydrates and fatty acids, respectively. This was the starting point of a dramatic scientific process that, within a century, led to the definition of the main biochemical pathways of intermediate metabolism. These include glycolysis (Meyerhof 1921, 1924), the Krebs' 
cycle (Krebs and Kornberg 1957), the beta-oxidation of fatty acids (Beinert 1963), and the oxidative phosphorylation in the electron transport chain (Mitchell 1979). Incidentally, Otto Fritz Meyerhof (glycolysis), Hans Krebs (1900-1981) (Krebs' cycle), and Peter Mitchell (1920-1992) (oxidative phosphorylation) were awarded the Nobel Prize for Physiology or Medicine in 1922, 1953, and 1978, respectively.

We also note that there was no discovery of metabolic pathways made by an isolated genius who unveiled the unknown. Meyerhof, Krebs, Beinert, and Mitchell synthesized in comprehensive theories complex metabolic processes, the development of which lasted decades and integrated the work of many scientists from several laboratories, who defined the single steps and described the various chemical components of each pathway. The ensemble of these pathways set the biochemical basis of oxidative metabolism, and provided an exhaustive explanation of the links between substrates (fuel) and oxygen, and thus between $\dot{V} \mathrm{O}_{2}$ and rate of carbon dioxide production $\left(\dot{\mathrm{V} C \mathrm{O}_{2}}\right)$. Those who are interested in the historical details of that epopee can refer to a bunch of reviews on these topics (see e.g. Ghisla 2004; Kornberg 2000; Krebs 1970; Mitchell 2004, 2011; Racker 1983).

By the second half of the nineteenth century, the physiological community was strongly convinced that the behaviour of all living things, muscles included, was amenable to physical and chemical laws. In this cultural climate, Hermann von Helmholtz (1821-1894) was the first who showed that the law of conservation of energy can be applied to living organisms (Helmoltz 1847), a hypothesis later supported more in detail by Danilewski (1880) and by Rubner (1894) in animals, and then by Atwater (1904) in man. The hypothesis that muscle is tantamount to a heat engine was supported by Mayer (1845), and later by Engelmann (1895). In contrast, Adolf Fick (1829-1901) rejected it, since the observed efficiency of human and horse muscles (20-25\%), as he rightly pointed out, would require temperature gradients physiologically unacceptable, on a heat engine hypothesis (Fick 1893).

Meanwhile, structural studies on muscles were carried out in what we can now define as the golden age of optical microscopy. Ranvier (1873) recognized the existence of red and white muscles with different morphological and physiological characteristics. Engelmann (1878) carefully described striation of muscle. Kölliker (1888) provided convincing evidence of the existence of myofibrils. At the dawn of the twentieth century, numerous studies on the energetics of muscular exercise in man had been carried out, along the path opened by the pioneering work of Lavoisier. The resulting conclusions by several authors (Chauveau and Kaufmann 1887; Chauveau and Tissot 1896; Heidenhain
1864; Heinemann 1901; Kölliker 1888; Pettenkofer and Voigt 1866; Zuntz 1901) on the fuel of choice, the energy expenditure, and the structure of muscle are closer to the currently accepted ones than we could have imagined, and represent the solid foundation for the revolution engendered by Meyerhof and Hill.

\section{A turning point: Hill and Meyerhof's theory}

After Fletcher and Hopkins (1907) had demonstrated that lactic acid, an end-product of glycolysis, accumulates in contracting muscles, Hill and Meyerhof, who shared the 1922 Nobel Prize for Physiology or Medicine, condensed the remarkable evidence collected by Fletcher and Hopkins (1907, 1917), and by the two of them separately (Hill 1913, 1916, 1922; Meyerhof 1920, 1921, 1922), in the first modern theory of the energetics of muscular contraction, currently incorporated under the term "Hill and Meyerhof's theory".

According to this theory, the primary energy source for muscle contraction is the oxidation of glycogen to lactic acid through glycolysis, regardless of the availability of oxygen. $\dot{V} \mathrm{O}_{2}$ intervenes only during recovery, when glycogen is resynthesized. Meyerhof $(1922,1924)$ assumed that during recovery, id est in aerobic conditions, about a quarter of the lactate produced during contraction is oxidized, thus yielding the energy for the resynthesis of the remaining threequarters to glycogen. An analysis of the time course of the $\dot{V} O_{2}$ at the onset of, during, and in the recovery after moderate exercise, prompted Hill et al. (1924) to introduce the term "oxygen debt". By this, they defined the amount of oxygen utilised in the recovery after exercise for the resynthesis to glycogen of part of the lactic acid accumulated in the contracting muscles during the preceding exercise period.

When Hill and Meyerhof formulated their theory of the energetics of muscle contraction, the existence of lactic acid had been known for more than a century. Carl Wilhelm Scheele isolated it from sour milk as impure brown syrup and it was recognized as an important chemical constituent of living organisms. Johannes Wislicenus (1835-1902) established its structure in 1873 (Wislicenus 1873). Then, Andersson (1933) described the structure of the enzyme catalysing the reduction of pyruvate to lactate, which he called lactate dehydrogenase.

Hill and Meyerhof's theory did not establish a clear distinction between aerobic and anaerobic metabolism during exercise. They considered the energy balance during muscle contraction an essentially anaerobic process, whereas the energy balance during recovery appeared as an aerobic process, for $\dot{V} O_{2}$ was necessary for glycogen synthesis. The cycle was closed by the combination of both processes. 
However, as time went by, the continuous evolution of biochemical knowledge provided numerous data that did not fit in the above picture:

a) Embden and Lawaczeck (1922) and Stella (1928) showed that the concentration of inorganic phosphate (Pi) in muscles increases during a series of contractions.

b) Eggleton and Eggleton (1927a, b), Fiske and Subbarow (1927, 1928), and Nachmanson (1928) identified phosphocreatine ( $\mathrm{PCr}$ ) and showed that its concentration in muscle decreases during contraction.

c) Lohmann (1928) isolated a new type of organic phosphate, adenosine-tri-phosphate (ATP), and showed that it is also present in muscle.

d) Lundsgaard (1930a, b) demonstrated that anoxic muscles poisoned with iodoacetic acid, which prevents lactate formation, can contract repeatedly, albeit for a limited time, without lactic acid accumulation.

Hill realised that the discovery of phosphates could undermine his theory and, as a scientist, accepted the risk of refutation. Therefore, he wrote an astonishing review (Hill 1932), wherein, from a "loser" perspective, he predicted a "revolution" in muscle physiology and tried to defend his theory by integrating the phosphates in it. However, contrary to the expectations of Hill's review, a masterpiece of humility, and intellectual honesty, it was not phosphate that killed the Hill and Meyerhof's theory. Phosphates could in principle be accommodated in it, by simply taking them as the link between the biochemical pathways and the contractile unit in muscle fibres. In fact, Rodolfo Margaria (1901-1983) actioned the guillotine and his concept of the alactic oxygen debt hammered the nail in the coffin of Hill and Meyerhof's theory.

\section{Margaria's refutation of Hill and Meyerhof's theory}

Margaria et al. (1933) showed that, over a large range of running speeds in humans, blood lactate concentration does not increase appreciably above resting, thus indicating that no lactate production, as they said, or, more correctly, no lactate accumulation, as we would say nowadays, has occurred during exercise. Hence, under those experimental conditions, the oxygen debt paid in the recovery period could not be attributed to lactate removal. Therefore, it was defined "alactic" oxygen debt and attributed to the resynthesis of the amount of "phosphagen" split at the onset of exercise. This finding effectively refuted Hill and Meyerhof's theory.

The study by Margaria et al. (1933) is the cornerstone of the theory that, after 90 years, is still considered to provide a substantially correct view of the energetics of muscle contraction and muscular exercise: the hydrolysis of phosphagen as the primary mechanism yielding the necessary amount of energy supporting muscle contraction and mechanical power generation. Optimally, and this is indeed the case during the exercise steady state, the hydrolysis of phosphagen and its resynthesis at the expense of oxygen consumption must proceed at the same rate. Conversely, at the beginning of exercise, because of the inertia of metabolic processes activation, a fraction of the phosphagen split during muscle contraction cannot be resynthesized; it will be only during recovery after exercise, at the expense of the "alactic" oxygen debt.

It is noteworthy that, when Margaria et al. (1933) published their study, ATP and PCr were not yet functionally separated, but were still lumped together under the term "phosphagen". The functional separation of ATP and PCr occurred one year later, when Lohmann (1934) proposed ATP to be the key molecule, necessary for muscular contraction. His view was better focused, and thus reinforced, in 1939, when Engelhardt and Lyubimova (1939) discovered the ATPase activity of myosin. Their discovery bridged the gap between structure and function, so that the energy liberation from ATP splitting could be accommodated in the subsequent sliding filament theory of muscle contraction (see e.g. Huxley 1957, 1974).

The refutation of Hill and Meyerhof's theory by Margaria et al. (1933) and the subsequent creation of the concept of the alactic oxygen debt gave origin to a huge amount of work on the energetics of muscular exercise in humans. To briefly summarize the most fundamental concepts, pertinent to this review, Margaria first wrote of different, yet concomitant metabolic pathways for ATP resynthesis during exercise, namely aerobic metabolism, sustaining moderate intensity exercise for long periods of time, the epiphenomenon of which is $\dot{V} O_{2}$, and anaerobic metabolism, sustaining explosive exercise for short periods of time. Later, to include the resynthesis of ATP from PCr hydrolysis in the picture, he splitted the concept of anaerobic metabolism in two: anaerobic lactic metabolism, represented by the rate of lactate accumulation in blood, and anaerobic alactic metabolism, implying ATP resynthesis from $\mathrm{PCr}$ without lactate accumulation. The latter accounts for explosive efforts of few seconds duration. In his view (Margaria 1968), each metabolism was characterised by a maximal capacity-maximal amount of energy that it can provide - and by a maximal power. Maximal oxygen consumption was identified as the maximal power attained by aerobic metabolism. He established the concept of an energy equivalent of blood lactate accumulation (Margaria et al. 1963), and used it to define the power at which the maximal rate of blood lactate accumulation (in fact, he wrote production...) is attained as the maximal lactic power (Margaria et al. 1964). 


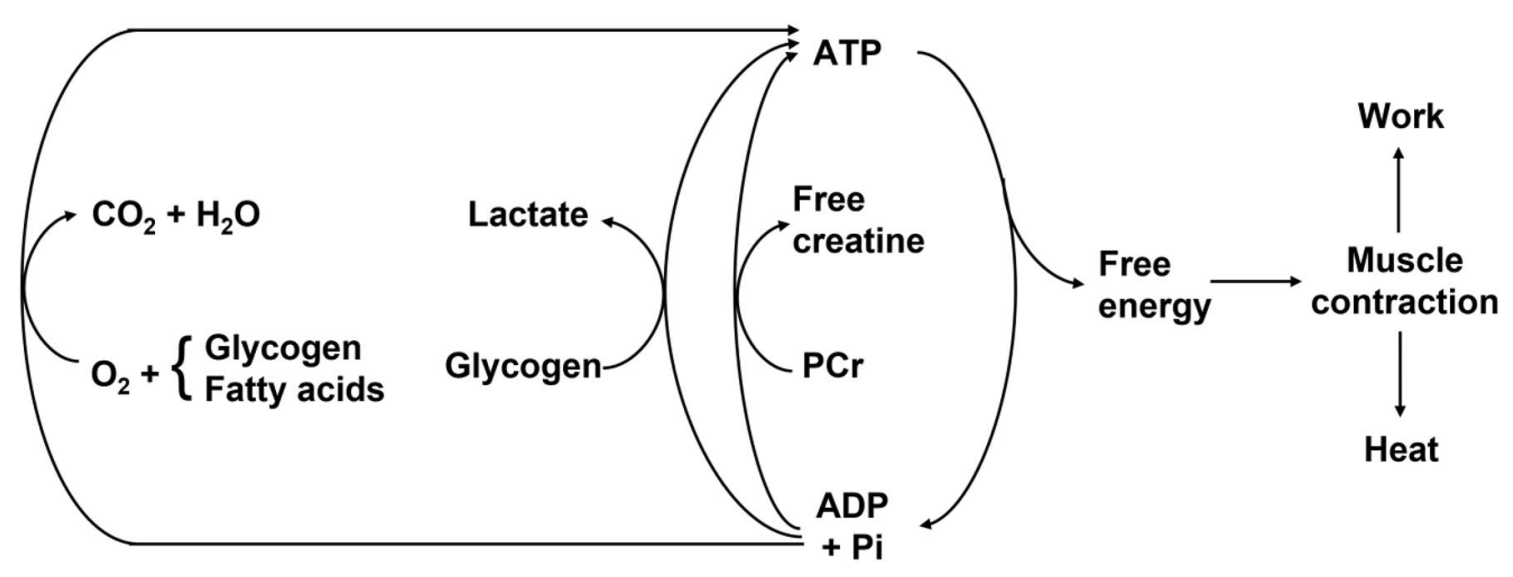

Fig. 1 Schematic representation of the energetics of muscular exercise. Adenosine triphosphate (ATP) hydrolysis provides the free energy that contracting muscles use to produce work and heat. ATP

Margaria was convinced, and rightly enough in our view, that there must be a relationship of direct proportionality between the epiphenomenal measurable variables characterising each metabolism $\left[\dot{V} O_{2}\right.$ for aerobic metabolism, rate of increase in blood lactate concentration $(\dot{\mathrm{La}})$ for anaerobic lactic metabolism, and rate of decrease in muscle $\mathrm{PCr}$ concentration $(\mathrm{PCr})$ for anaerobic alactic metabolism], and the rate of energy delivery (metabolic power, $\dot{E}$ ). Thus, each metabolism can indeed be defined by its maximal power and capacity and by its energy equivalent (di Prampero 1981; di Prampero and Ferretti 1999; Ferretti 2015).

Margaria's energetic vision of exercise physiology led to the analysis of the exercise transient in terms of oxygen deficit, which we now consider as consisting of an obligatory (former Margaria's alactic oxygen debt) and a facultative ("early lactate" accumulation and changes in oxygen stores) component (di Prampero 1981). The latter intervenes only when the former is unable, for any reason, to ensure the energy balance of the oxygen deficit. The alactic oxygen deficit is a physiological necessity, as long as a reduction of muscle $\mathrm{PCr}$ concentration determines the activation of the glycolytic pathway, and thus the acceleration of the entire oxidative pathways leading to the increase of $\dot{V} O_{2}$. Thus, PCr, and its counterpart, free creatine, are key molecules for coupling ATP resynthesis to ATP consumption during muscular contraction (di Prampero and Margaria 1968; di Prampero et al. 2003; Mader 2003; Mahler 1985). These concepts will be better detailed in Sect. "The Energetics of the Oxygen Deficit".

All developments subsequent to the work of Margaria et al. (1933), especially within the physiological school that he initiated, had the remarkable property of being fully compatible with the general principles of thermodynamics. The successors of Margaria in Milano kept this concept resynthesis is ensured by the indicated pathways. ADP, adenosine-diphosphate, $\mathrm{Pi}$, inorganic phosphate, $\mathrm{PCr}$, phosphocreatine [Modified after di Prampero (1981)]

very clear in their mind, and the experiments that they conceived to test Margaria's energetic theory were driven by a thermodynamic vision. The energetics of human locomotion has also been treated within the boundaries of thermodynamic compatibility. The energetics of muscular exercise and of human locomotion has been analysed and discussed in numerous reviews and books ( $⿱$ Astrand et al. 2003; Brooks 2000, 2012; di Prampero 1981, 1986, 2000, 2015; di Prampero and Ferretti 1999; Ferguson et al. 2018; Ferretti 2014, 2015; Grassi 2000, 2003; Jones and Poole 2005; Lacour and Bourdin 2015; Poole and Jones 2012; Poole et al. 2016, 2021; Taylor and Heglund 1982; Whipp and Ward 1982, 1990; Zamparo et al. 2020), which the readers are referred to for more detailed analysis.

The large volume of data collected and of theoretical analyses formulated after the paper by Margaria et al. (1933) have led to the creation of a general theory of the energetics of muscular exercise, which we mostly owe to the physiological school that Rodolfo Margaria established in Milano after World War II. The first formulation of this theory dates back to 1968 (di Prampero and Margaria 1968); its first comprehensive summary was published in 1981 (di Prampero 1981). This general theory, summarized in Fig. 1, can be condensed in algebraic form as follows (di Prampero 1981; Ferretti 2015):

$\dot{E} \propto \overleftarrow{A \dot{T} P}=\overrightarrow{A \dot{T} P}=c \dot{V} O_{2}+b \dot{L} a+a P \dot{C} r$,

where the metabolic power $\dot{E}$ represents the overall rate of metabolic energy liberation, $\overleftrightarrow{A \dot{T} P}$ and $\overrightarrow{A \dot{T} P}$ are the rates of ATP hydrolysis and resynthesis, respectively, $\dot{L} a$ is the rate of lactate accumulation in blood, and $P \dot{C} r$ is the rate of $\mathrm{PCr}$ drop in the working muscle fibres. The three constants $a, b$, and $c$ are proportionality constants indicating the moles of ATP resynthesized, respectively, by a mole of hydrolysed 
PCr, a mole of lactate accumulated, and a mole of oxygen consumed. The Lohmann's reaction tells that $a$ is equal to 1; $c$ corresponds to the $P / O_{2}$ ratio and is equal to 6.17 for complete glycogen oxidation into glycolysis, Krebs cycle, and oxidative phosphorylation. For constant $b$, corresponding to the $P / L a$ ratio (moles of phosphate released per mole of lactate formed by glycolysis), the situation is a bit more complex (see di Prampero 1981; di Prampero and Ferretti 1999; Ferretti 2015). Notwithstanding, the data collected so far indicate a mean value for $b$ ranging between 1.25 and $1.05 \mathrm{~mol}$ of phosphate per mole of lactate accumulated, which is slightly less than the value of 1.5 predicted from stoichiometry.

As shown in Fig. 1, the general theory of the energetics of muscular exercise considers muscle as a biological engine, in which chemical energy is transformed into mechanical work and heat. The ultimate step of this energy transformation is provided by ATP hydrolysis in the cross-bridge cycle. The low ATP concentration in muscle (on average $5 \mathrm{mmol}$ per $\mathrm{kg}$ of wet muscle) requires continuous ATP resynthesis, which we owe to the ensemble of reactions comprised in the concept of intermediate metabolism and synthetized in Eq. (2). Three basic metabolic pathways for ATP resynthesis appear, each corresponding to a precise physiological concept: aerobic metabolism, anaerobic lactic metabolism, and anaerobic alactic metabolism. Aerobic metabolism includes the oxidation of glycogen and fatty acids to pyruvate and acetate, respectively, and their introduction into the Krebs' cycle, which feeds oxidative phosphorylation. Anaerobic lactic metabolism includes the degradation of glycogen to pyruvate, with subsequent reduction of pyruvate to lactate by the action of lactate dehydrogenase. It occurs when the rate of energy transformation in glycolysis exceeds that of oxidation of pyruvate in the Krebs' cycle. Anaerobic alactic metabolism includes resynthesis of ATP through the Lohmann reaction and hydrolysis of immediately available ATP.

$\dot{\mathrm{La}}$ and $\mathrm{PC} r$ are intrinsic muscular phenomena, which are fully separated from the respiratory system. Of course $\dot{L} a=0 \mathrm{mmol} \mathrm{s}^{-1}$ if lactate production equals lactate removal by muscle fibres and by other organs. The same is the case for $\mathrm{PC}$, if $\mathrm{PCr}$ remains constant, as occurs when aerobic metabolism can resynthesize ATP at a rate sufficient to cope with ATP hydrolysis during muscle contraction. This condition is certainly attained in humans at rest and during moderate exercise, when $\dot{V} \mathrm{O}_{2}$ reaches a steady level, which is maintained for a long time. Under these steady-state conditions, there is neither accumulation of lactate in blood nor changes in muscle $\mathrm{PCr}$ concentration, so that the terms $b \dot{\mathrm{La}}$ and $a P C$ of Eq. (2) are nil, the metabolism is purely aerobic, and Eq. (2) reduces to

$\dot{E} \propto \overleftarrow{A \dot{T} P}=\overrightarrow{A \dot{T} P}=c \dot{V} O_{2}$
When Eq. (3) applies, all the metabolic power sustaining body functions at rest, plus the mechanical work for exercise, derives from aerobic energy sources, $\dot{V} O_{2}$ is proportional to $\dot{E}$, and ATP resynthesis through the overall biochemical pathway of aerobic metabolism equals ATP consumption. In a steady-state condition, $\dot{E}$ and thus $\dot{V} O_{2}$ stay invariant in time.

The tight matching of $\dot{V} O_{2}$ with $\dot{E}$, however, can be maintained only if there is a tight matching between $\dot{V} O_{2}$ and the oxygen flow along the respiratory system $\left(\dot{V}_{R} O_{2}\right)$, inasmuch as the $\dot{V} O_{2}$ can be sustained only by taking oxygen from ambient air and conveying it to cells (mostly muscle fibres at exercise) along the respiratory system. In other terms, cellular respiration must be strictly coupled to $\dot{V}_{R} O_{2}$, which occurs if, and only if

$\dot{V} O_{2}=\dot{V}_{R} O_{2}$.

In the next paragraphs, we discuss how the conditions summarized by Eq. (4) are attained and maintained in the body. We first introduce the steady state concept and its relation to the respiratory system, and describe the quantitative relationships that characterise it. Then, we discuss how the equilibrium is maintained when $\dot{V} O_{2}$ increases, as at exercise, we analyse the cardiopulmonary responses to exercise at steady state, and synthetize the interrelated phenomena along the respiratory system by which a steady $\mathrm{VO}_{2}$ is maintained during exercise.

\section{The steady-state concept}

Bock et al. (1928) defined the steady-state concept for the first time. According to them, a human is in steady-state condition when: $\dot{V} O_{2}$ and $\dot{V} C O_{2}$ are invariant, the carbon dioxide eliminated through the mouth is produced only by metabolism, there is a steady heart rate $\left(f_{H}\right)$, and the milieu intérieur is essentially stable. This definition of steady state implies that

1) Equation (4) holds at steady state.

2) $\dot{V}_{R} O_{2}$ takes the same value at any level along the respiratory system, i.e., in the alveoli, across the systemic circulation and across the alveolar-capillary barrier.

3) The outflow of carbon dioxide $\left(\dot{V}_{R} \mathrm{CO}_{2}\right)$ is the same at any level along the respiratory system, i.e., across the systemic circulation, across the alveolar-capillary barrier and in the alveoli, and is equal to $\dot{\mathrm{V} C \mathrm{O}_{2}}$.

If the preceding points are correct, it necessarily follows that the respiratory quotient determined at the lungs $\left(R Q_{L}\right)$ or gas exchange ratio, defined as the ratio between $\dot{V}_{R} C O_{2}$ and $\dot{V}_{R} O_{2}$-is equal to the respiratory quotient determined at 
any other level along the respiratory system. Consequently, $R Q_{L}$ is also equal to the metabolic respiratory quotient $\left(R Q_{M}\right)$, id est the ratio between the moles of carbon dioxide produced and the moles of oxygen consumed at the cellular level.

Taking the above three points as axioms, several developments in the analysis of the quantitative relationships describing gas flows in the respiratory system at rest and exercise have been pursued. Before entering into these details, however, it is necessary to point out that the steadystate concept is a mental creation of some brilliant physiologists, yet represents an oversimplification of real life. This type of steady state has been reasonably approximated in the laboratory, for instance at rest and during moderate exercise at constant power. Notwithstanding, even at steady state, the respiratory system shows oxygen flow discontinuities, heterogeneities and spontaneous variations, depending on the level of organisation, macroscopic or microscopic, at which we operate.

At the macroscopic level, pulmonary ventilation occurs in a cul de sac, so that, contrary to what happens in birds' parabronchi, air must enter and quit the lungs through a common path, consisting of the airways. Such a structural arrangement implies that (i) air inhalation (inspiration) and exhalation (expiration) occur necessarily in alternate manner: no continuous steady air flow is possible in such a system, and (ii) a fraction of the inhaled air cannot attain the alveoli, but remains trapped in the airways. This fraction cannot participate in alveolar gas exchange, and thus, its volume is called the dead space volume (Bohr 1891; Krogh and Lindhard 1913a, 1917). Moreover, the heart alternates systoles and diastoles, so that pressure varies continuously inside the ventricles and in the aorta. The opening and closing of heart valves occur synchronous to pressure variations. Therefore, blood flow in the heart and in the aorta is necessarily discontinuous. Discontinuities in ventilation and in total blood flow carry along oscillations both in oxygen and in carbon dioxide flows. We have then to consider that there is a spontaneous variability of respiratory and cardiac rhythms, related to mechanical and neural control mechanisms (Cottin et al. 2008; Perini and Veicsteinas 2003). This being the case, $\dot{V}_{R} O_{2}$ and $\dot{V}_{R} C_{2}$ at steady state are not continuous invariant flows: the steady-state $\dot{V}_{R} \mathrm{O}_{2}$ and $\dot{\mathrm{V}}_{R} \mathrm{CO}_{2}$ are invariant integral means of flows that are highly variable in time, at several levels even discontinuous.

At the microscopic level, blood flow is pulsatile in lung capillaries, because of their heterogeneous recruitment in space and time, and because of the effects of the rhythmic activity of the heart and the lungs on lung capillary pressure (Baumgartner et al. 2003; Clark et al. 2011; Heinonen et al. 2013; Roy and Secomb 2019; Tanabe et al. 1998). Heterogeneity of lung capillary blood flow may be less at exercise, due to simultaneous recruitment of a larger number of lung capillaries to sustain the higher pulmonary blood flow.

Similar heterogeneities in capillary blood flow were demonstrated also in contracting skeletal muscles, both in space and in time (Armstrong et al. 1987; Ellis et al. 1994; Heinonen et al. 2007; Marconi et al. 1988; Piiper et al. 1985). Heterogeneous distribution of muscle blood flow was found also in non-contracting muscles of exercising humans (Heinonen et al. 2012). Contracting muscle fibres are likely unperfused, because they generate pressure, which compresses and closes muscle capillaries from outside as a Starling resistor. If this is so, only relaxed muscle fibres would be perfused, so that muscle fibre oxygenation takes place during relaxation, not during contraction. In this case, the alternate recruitment of neighbouring motor units is a physiological necessity, entailing heterogeneity of muscle blood flow distribution during exercise (Cano et al. 2015; Goulding et al. 2021). Nevertheless, capillaries themselves are tethered to adjacent myocytes by collagenous struts, which, when pulled, exert a force acting in the opposite direction (Abovsky et al. 1996; Borg and Caulfield 1980; Caulfield et al. 1985). In both skeletal and cardiac muscle capillaries, this force helps keep capillaries open. However, during intense contractions, capillary flow may cease when arterioles and venules collapse. This means that, at least during short contractions of the surrounding fibres, red blood cells, which are retained in the closed capillaries, may keep exchanging gases.

Finally, we note that, on a longer time scale, also $R Q_{M}$ can change, if substrate utilisation shifts between glycogen and fatty acids as energy sources. This occurs, for instance, during very long light exercise, when glycogen stores are close to exhaustion, so that fatty acid oxidation represents a progressively larger fraction of the overall aerobic metabolism (Fernandez-Verdejo et al. 2018; Galgani et al. 2008, 2012).

\section{Quantitative relationships at steady state}

\section{Of two mass balance equations}

Keeping the three axioms listed in the previous section in mind, let us start from a definition of $\dot{V}_{R} \mathrm{O}_{2}$ and $\dot{V}_{R} \mathrm{CO}_{2}$ as a difference between a flow in and a flow out for the gas at stake, providing the gas flow exchanged in the lung alveoli and in peripheral capillaries. ${ }^{1}$ This definition implies that

\footnotetext{
1 This definition implies that in blood, $\dot{V}_{R} \mathrm{O}_{2}$ and $\dot{V}_{R} \mathrm{CO}_{2}$ are not the flow of the respective gases in arterial or in mixed venous blood, but the difference between these two flows; analogously, in the lungs, $\dot{V}_{R} O_{2}$ and $\dot{V}_{R} C O_{2}$ are not the flow of the respective gases in inspired or in expired air, but the difference between these two flows.
} 
$\dot{V}_{R} \mathrm{O}_{2}$ and $\dot{\mathrm{V}}_{R} \mathrm{CO}_{2}$ can be obtained by mass balance equations. Fick (1870) described the first equations of this type, which summarize what we call in his honour the Fick principle, and which are expressed in the following form, respectively for oxygen and carbon dioxide:

$\dot{V}_{R} O_{2}=\dot{Q} C_{a} O_{2}-\dot{Q} C_{\bar{v}} O_{2}=\dot{Q}\left(C_{a} O_{2}-C_{\bar{v}} O_{2}\right)$,

$\dot{V}_{R} \mathrm{CO}_{2}=\dot{Q} \mathrm{C}_{a} \mathrm{CO}_{2}-\dot{Q} C_{\bar{v}} \mathrm{CO}_{2}=\dot{Q}\left(\mathrm{C}_{a} \mathrm{CO}_{2}-\mathrm{C}_{\bar{v}} \mathrm{CO}_{2}\right)$,

where $\dot{Q}$ is total blood flow, or more commonly cardiac output, $\mathrm{C}_{a} \mathrm{O}_{2}$ and $\mathrm{C}_{\bar{v}} \mathrm{O}_{2}$, and $\mathrm{C}_{a} \mathrm{CO}_{2}$ and $\mathrm{C}_{\bar{v}} \mathrm{CO}_{2}$ are the oxygen and carbon dioxide concentrations in arterial and mixed venous blood, respectively. In fact, Eqs. $(5 a)^{2}$ and (5b) define, respectively, the volume of oxygen that leaves the blood in peripheral capillaries to be consumed in cells and the volume of carbon dioxide produced by metabolism that leaves the cells to be removed through the respiratory system, per unit of time.

Along the same line, Geppert and Zuntz (1888) defined $\dot{V}_{R} O_{2}$ as the difference between inspired and expired oxygen flows, as follows:

$\dot{V}_{R} O_{2}=\dot{V}_{I} F_{I} O_{2}-\dot{V}_{E} F_{E} O_{2}$,

$\dot{V}_{R} C O_{2}=\dot{V}_{I} F_{I} C O_{2}-\dot{V}_{E} F_{E} C O_{2}$,

where $\dot{V}_{I}$ is the total inspired air flow, or inspiratory ventilation, $\dot{V}_{E}$ is the total expired air flow, or expiratory ventilation, $F_{I} O_{2}$ and $F_{E} O_{2}$ are the oxygen fractions in inspired and expired air, respectively, and $\mathrm{F}_{I} \mathrm{CO}_{2}$ and $\mathrm{F}_{E} \mathrm{CO}_{2}$ are the carbon dioxide fractions in inspired and expired air, respectively. Further developments around Eq. (6a), accounting for the fact that $R Q_{L}=R Q_{M}$ is less than 1, and thus, $\dot{V}_{E}<\dot{V}_{I}$, in a variety of steady-state conditions, can be found in Otis (1964) and led to the following equation for the calculation of $\dot{V}_{R} O_{2}$, when $\dot{V}_{I}$ cannot be measured:

$\dot{V}_{R} O_{2}=\dot{V}_{E}\left[\frac{\left(1-F_{E} O_{2}-F_{E} C O_{2}\right)}{F_{I} N_{2}} F_{I} O_{2}-F_{E} O_{2}\right]$,

where $F_{I} N_{2}$ is the nitrogen fraction in inspired air.

\section{Maintaining the equilibrium in the lungs}

Since in respiration physiology, $\mathrm{F}_{\mathrm{I}} \mathrm{CO}_{2}$ is generally considered to be negligible (in fact, average $F_{I} \mathrm{CO}_{2}=0.000415$ in

\footnotetext{
${ }^{2} \dot{V}_{R} O_{2}$ and $\dot{V}_{R} C O_{2}$ are flows along the respiratory system, oriented in opposite directions. Equations (5a) and (5b) clearly show that, since $\mathrm{C}_{a} \mathrm{O}_{2}>\mathrm{C}_{\bar{v}} \mathrm{O}_{2}$, whereas $\mathrm{C}_{a} \mathrm{CO}_{2}<\mathrm{C}_{\bar{v}} \mathrm{CO}_{2}, \dot{V}_{R} \mathrm{O}_{2}$ results positive and $\dot{V}_{R} C_{2}$ turns out negative. This implies that $R Q_{L}$ is negative as well. This concept carries along a notation differing from the usual notation, wherein the modulus of these flows is used.
}

dry air), the first term of the right-hand branch of Eq. (6b) can be omitted. Moreover, at steady state, $\dot{\mathrm{V}}_{\mathrm{R}} \mathrm{CO}_{2}$ is equal in expired as in alveolar air. Thus, in steady-state condition, Eq. (6b) can be rewritten as follows:

$\dot{V}_{R} C O_{2}=-\dot{V}_{E} F_{E} C O_{2}=-\dot{V}_{A} F_{A} C O_{2}=-\dot{V}_{A} \frac{P_{A} C O_{2}}{P_{B}-47}$,

where $\dot{V}_{A}$ is alveolar ventilation, $P_{A} \mathrm{CO}_{2}$ is the partial pressure of carbon dioxide in alveolar air, $P_{B}$ is the barometric pressure, and $47 \mathrm{mmHg}$ is the water pressure in the alveoli, assuming alveolar air to be at $37^{\circ} \mathrm{C}\left(310^{\circ} \mathrm{K}\right)$ and saturated with water. Equation (8) tells that at any given $P_{B}$ and $\dot{V}_{R} C O_{2}, \dot{V}_{A}$ is inversely proportional to $\mathrm{P}_{A} \mathrm{CO}_{2}$, whereas at any given $\mathrm{P}_{A} \mathrm{CO}_{2}$, it is directly proportional to $-\dot{V}_{R} C O_{2}$. The linear relationship between $\dot{V}_{A}$ and $-\dot{V}_{R} C O_{2}$ in "standard" steady-state acid-base conditions $(\mathrm{pH}=7.40$, arterial carbon dioxide partial pressure, $P_{a} \mathrm{CO}_{2}=40 \mathrm{mmHg}$; bicarbonate concentration $\left.=25 \mathrm{mEq} \mathrm{L}^{-1}\right)$ lies on the $\mathrm{P}_{\mathrm{A}} \mathrm{CO}_{2}$ isopleth for $40 \mathrm{mmHg}\left(\mathrm{P}_{A} \mathrm{CO}_{2}=\mathrm{P}_{a} \mathrm{CO}_{2}\right)$, which implies a $\dot{V}_{A} / \dot{V}_{R} C O_{2}$ equal to $-21.6{ }^{3}$ At exercise, the increase in $\dot{V}_{A}$ is proportional to that in $-\dot{V}_{R} C O_{2}$, a condition that has been called hyperpnoea: the acid base conditions are kept invariant and equal to the resting ones.

This is so as long as there is no continuous blood lactate accumulation $(b \dot{L a}=0 \mathrm{~W})$, and thus, Eq. (3) applies. This is the case at rest, as well as during moderate aerobic exercise (Ferretti 2015; Poole et al. 2016). A continuous blood lactate accumulation occurs and affects $\mathrm{pH}$ regulation at powers above the critical power (Jones et al. 2008). In top-level marathon runners, this occurs at powers higher than $90 \%$ of the maximal aerobic power (Tam et al. 2012).

Above these intensities, although the exercise is still submaximal, lactate keeps increasing in blood, the term $b \dot{L} a$ of Eq. (2) becomes positive and $\mathrm{pH}$ is not sufficiently buffered. Hyperventilation occurs, and is superimposed to hyperpnoea, and the $\dot{V}_{A}$ versus $-\dot{V}_{R} \mathrm{CO}_{2}$ relationship bends upward, toward isopleths for lower $P_{A} \mathrm{CO}_{2}$ and more negative $\dot{V}_{A} / \dot{V}_{R} \mathrm{CO}_{2}$. Moreover, the so-called slow component of the $\dot{V}_{R} O_{2}$ kinetics appears, so that $\dot{V}_{R} O_{2}$ keeps increasing with time (Ferretti 2015; Poole and Jones 2012; Poole et al. 1988, 1994). Steady-state conditions are disrupted, as is the tight matching between $\dot{V} O_{2}$ and $\dot{V}_{R} O_{2}$. Nevertheless, even in the severe exercise domain (above the critical power), type I muscle fibres can still consume the oxygen that the respiratory system is able to deliver to them. Concerning type II fibres, neither nuclear magnetic resonance (Richardson

\footnotetext{
${ }^{3}$ Since $\dot{V}_{R} \mathrm{CO}_{2}$ is in STPD and $\dot{V}_{A}$ in BTPS, expressing both variables in standard condition, so that $F_{A} \mathrm{CO}_{2}=\frac{P_{A} \mathrm{CO}_{2}}{P_{B}-47}$, yields $\frac{\dot{V}_{A[B T P S]}}{\dot{V}_{R} C O_{2[S T P D]}}=-\frac{P_{B}-47 \mathrm{mmHg}}{P_{A} C O_{2}} \frac{760 \mathrm{mmHg}}{273^{\circ} \mathrm{K}} \frac{310^{\circ} \mathrm{K}}{P_{B}-47 \mathrm{mmHg}}=-\frac{863 \mathrm{mmHg}}{P_{A} C O_{2}}$. This means that, for $P_{A} C_{2}=40 \mathrm{mmHg}, \frac{\dot{V}_{A[B T P S]}}{\dot{V}_{R} \mathrm{CO}_{2[S T P D]}}=-\frac{863}{40}=-21.6$. This value does not vary at exercise, because $\dot{V}_{A}$ and $\dot{V}_{R} \mathrm{CO}_{2}$ increase in direct proportion.
} 


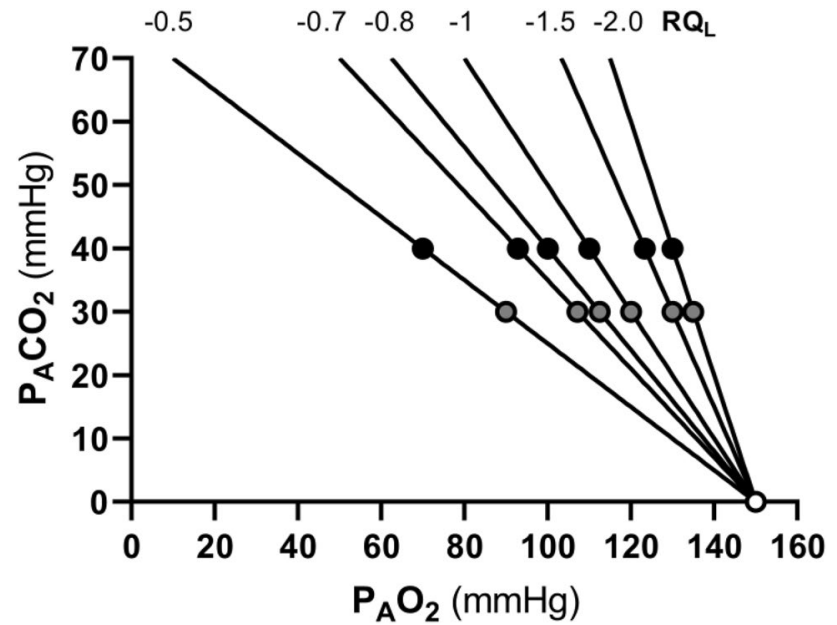

Fig. $2 \mathrm{O}_{2}-\mathrm{CO}_{2}$ diagram for alveolar air, wherein alveolar partial pressure of carbon dioxide $\left(\mathrm{P}_{\mathrm{A}} \mathrm{CO}_{2}\right)$ is plotted as a function of the alveolar partial pressure of oxygen $\left(\mathrm{P}_{A} \mathrm{O}_{2}\right)$. Six isopleths of pulmonary respiratory quotient $\left(R Q_{L}\right)$ are shown, converging on the $x$-axis at a value corresponding to the inspired partial pressure of oxygen at sea level (white dot), thus yielding the gas composition of ambient air. On each line, the points indicating alveolar air composition (black dots) and expired air composition (grey dots) at each $R Q_{L}$ are reported. Note that, for alveolar air, $P_{A} C_{2}$ is always at $40 \mathrm{mmHg}$ (regulated variable), independent of $R Q_{L}$. This necessarily implies that, the closer to zero is the $R Q_{L}$, the lower is the $P_{A} O_{2}$ [Modified after Ferretti et al. (2017)]

et al. 2015) nor cryomicrospectroscopy (Honig et al. 1984) provided any evidence that oxygen delivery is limited in the severe exercise domain: thus, the metabolic limit of these fibres may be set by their ability to consume oxygen.

If Eq. (6a) is rewritten in terms of $\dot{V}_{A}$, by subtracting dead space ventilation, and of partial pressures, by applying Dalton's law, and we divide the result by Eq. (8), we can compute $R Q_{L}$ as follows:

$R Q_{L}=\frac{-P_{A} C_{2}}{\left(P_{I} O_{2}-P_{A} O_{2}\right)}$,

where $P_{I} O_{2}$ and $P_{A} O_{2}$ are the oxygen partial pressures in inspired and alveolar air, respectively. The solution of Eq. (9) for $P_{A} O_{2}$ and $P_{A} \mathrm{CO}_{2}$ yields

$P_{A} O_{2}=P_{I} O_{2}-\frac{-P_{A} C O_{2}}{R Q_{L}}$

$P_{A} \mathrm{CO}_{2}=R Q_{L}\left(P_{A} O_{2}-P_{I} O_{2}\right)$.

Equations (10) and (11) represent two expressions of the so-called alveolar air equation (Fenn et al. 1946). Equation (11) tells that, if in steady-state condition, we plot $P_{A} \mathrm{CO}_{2}$ as a function of $P_{A} \mathrm{O}_{2}$ on a $x-y$ diagram, we obtain a linear relationship, the slope of which is equal to $R Q_{L}$ (thus,

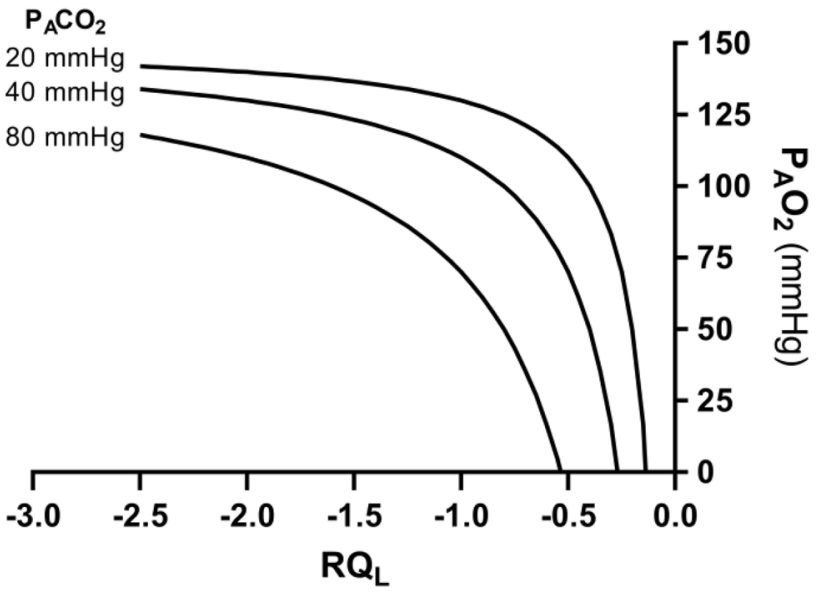

Fig. 3 A representation of three hyperbolic functions describing the relationship between the alveolar partial pressure of oxygen $\left(\mathrm{P}_{A} \mathrm{O}_{2}\right)$ and the pulmonary respiratory quotient $\left(R Q_{L}\right)$. The reported curves hold for an inspired partial pressure of oxygen of $150 \mathrm{mmHg}$ (horizontal asymptote) and for the indicated alveolar partial pressures of carbon dioxide, expressed in $\mathrm{mmHg}$ (curvature)

it is negative), and which has an intercept on the $x$-axis equal to $P_{I} O_{2}$, thus providing the composition of ambient inspired air $\left(P_{I} \mathrm{CO}_{2}=0 \mathrm{mmHg}\right)$. This graphical representation is the oxygen-carbon dioxide diagram $\left(\mathrm{O}_{2}-\mathrm{CO}_{2}\right.$ diagram $)$ for alveolar gases (Rahn and Fenn 1955) (Fig. 2), A family of isopleths for $R Q_{L}$, all converging on a given inspired air composition (e.g., $P_{I} O_{2}=150 \mathrm{mmHg}$ at sea level), are reported on the same figure.

It is a corollary of the steady-state axioms that, in fact, the only possible alveolar air compositions lie on the $R Q_{L}$ isopleth corresponding to the actual $R Q_{M} . R Q_{M}$ ranges between -0.8 at rest and -1.0 around the lactate threshold, the difference depending on the increasing fraction of energy derived from carbohydrate oxidation, as the exercise intensity, and thus the $\dot{V} O_{2}$, go up. Within this range of metabolic powers, the maintenance of the homeostatic equilibrium described above ensures tight coupling of respiration and metabolism. The invariance of $P_{A} \mathrm{CO}_{2}$ around the value of $40 \mathrm{mmHg}$ is ensured at the expense of $P_{A} O_{2}$, the value of which at steady state necessarily varies depending on $R Q_{L}$. These $P_{A} O_{2}$ variations are predictable from Eq. (10).

Equation (11) tells also that, if we plot $P_{A} O_{2}$ as a function of $R Q_{L}$, the relationship is described by a translated hyperbola, with a negative curvature (downward convexity) equal to $-P_{A} \mathrm{CO}_{2}$, and a horizontal asymptote equal to $P_{I} \mathrm{O}_{2}$. The relationship between $P_{A} O_{2}$ and $R Q_{L}$ for a $\dot{V}_{A} / \dot{V}_{R} C O_{2}$ of $-21.6\left(P_{A} \mathrm{CO}_{2}=40 \mathrm{mmHg}\right)$ at sea level $\left(P_{I} O_{2}=\right.$ $150 \mathrm{mmHg}$ ) is shown in Fig. 3. Obviously enough, a family of isopleths for $\dot{V}_{A} / \dot{V}_{R} C O_{2}=-21.6$ can be constructed, displaced downward if $P_{I} O_{2}$ decreases (hypoxia), upward if $\mathrm{P}_{I} \mathrm{O}_{2}$ increases (hyperoxia). Otherwise, for any given $\mathrm{P}_{I} \mathrm{O}_{2}$, 
when the $\dot{V}_{A} / \dot{V}_{R} C O_{2}$ decreases (becomes more negative) and the $\mathrm{P}_{\mathrm{A}} \mathrm{CO}_{2}$ decreases (hyperventilation), the curve becomes more convex; conversely, when the $\dot{V}_{A} / \dot{V}_{R} \mathrm{CO}_{2}$ goes up and the $P_{A} C_{2}$ increases (hypoventilation), the curve becomes less convex.

Variations of $P_{I} O_{2}$ in hypoxia and in hyperoxia have an impact also on the $\mathrm{O}_{2}-\mathrm{CO}_{2}$ diagram, in so far as the inspired air point is shifted leftward and rightward, respectively. Since the $R Q_{L}$ isopleths converge on the inspired air point, the entire family of these isopleths is displaced accordingly. Notwithstanding, as long as there is no hyperventilation due to hypoxaemic stimulation of peripheral chemoreceptors, the $\dot{V}_{A} / \dot{V}_{R} C O_{2}$ remains invariant at -21.6 , and thus, $P_{A} C_{2}$ stays equal to $40 \mathrm{mmHg} .{ }^{4}$ Conversely, when the hyperventilation induced by hypoxaemia intervenes, which is accentuated at a $\mathrm{P}_{a} \mathrm{O}_{2}$ lower than some $60 \mathrm{mmHg}$, the $\dot{V}_{A} / \dot{V}_{R} \mathrm{CO}_{2}$ and $P_{A} C_{2}$ decrease. For any given $R Q_{L}$, the alveolar gas composition is displaced downward and rightward along the corresponding $R Q_{L}$ isopleth, in the direction of the inspired air point. The $P_{A} O_{2}$ increases by an amount that is predictable from the alveolar air equation, based on the incurring $P_{A} \mathrm{CO}_{2}$ and $\mathrm{RQ}_{L}$.

If for each $P_{I} O_{2}$ value, and thus each $R Q_{L}$ isopleth, we connect all the points representing the steady-state alveolar air composition, we obtain a curve like that reported in Fig. 4, which Rahn and Fenn (1955) called the normal mean alveolar air curve. If no hypoxaemia occurs, this curve is parallel to the $x$-axis, indicating that $P_{A} \mathrm{CO}_{2}$ is independent of $P_{I} O_{2}$ in that segment of the curve, as long as $\dot{V}_{A} / \dot{V}_{R} C O_{2}$ $=-21.6$, and thus, the "standard" steady-state acid-base conditions are preserved. As the $P_{I} O_{2}$ becomes sufficiently low to determine a stimulation of the peripheral chemoreceptors, and thus hyperventilation, the $\dot{V}_{A} / \dot{V}_{R} C O_{2}$ decreases and the curve bends downward, so that $P_{A} \mathrm{CO}_{2}$ becomes lower, the stronger is hyperventilation, and the lower becomes the $\dot{V}_{A} / \dot{V}_{R} C O_{2}$.

\footnotetext{
${ }_{4}$ The extraordinary stability of $\mathrm{P}_{A} \mathrm{CO}_{2}$ and $\mathrm{P}_{a} \mathrm{CO}_{2}$ around $40 \mathrm{mmHg}$ throughout life has to do with the fact that the ventilatory response to carbon dioxide (actually to the $H+$ concentration in the cerebrospinal fluid) is a threshold phenomenon, with threshold at an $H+$ concentration compatible with $\mathrm{P}_{a} \mathrm{CO}_{2}=40 \mathrm{mmHg}$. The phenomenon is a consequence of how the acid-sensing ion channels interact with $H+$. Bound $H+$ is in dynamic equilibrium with free $H+$. When a sufficiently high number of $H+$ units are bound to the extracellular region of the channel, the transmembrane domain 2 of the channel undergoes conformational changes leading to channel opening. An influx of sodium ions, and thus cell depolarization, occur, leading to activation of voltage-gated calcium channels. The ASIC 1 and ASIC 2 channels are widely expressed in the ventro-lateral medulla. For details, see e.g. Hanukoglu (2017), Song et al. (2016) and Zha (2013). On the role of central chemoreceptors in regulating $\mathrm{pH}$ and $\mathrm{P}_{a} \mathrm{CO}_{2}$, see Guyenet (2014), and Nattie and Li (2012).
}

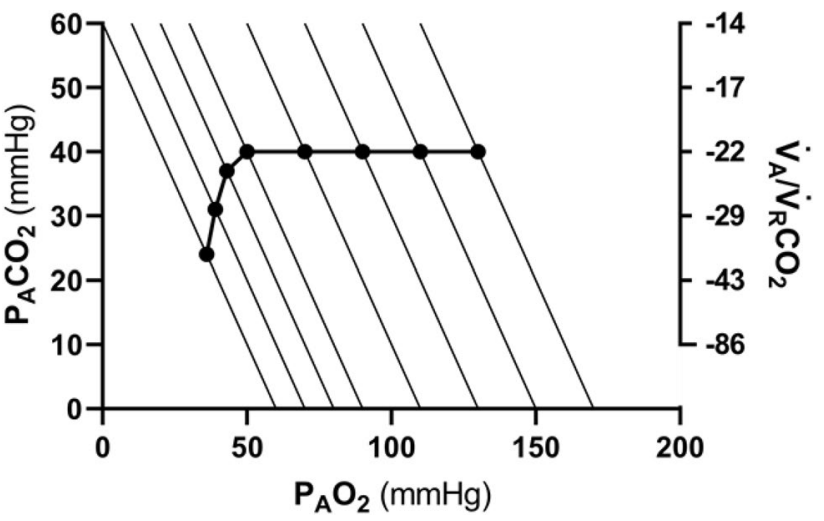

Fig. 4 On the $\mathrm{O}_{2}-\mathrm{CO}_{2}$ diagram for alveolar air, the thin parallel lines are isopleths for a lung respiratory quotient of -1.0 , which intercept the $x$-axis at different inspired air points. As the inspired partial pressure of oxygen $\left(\mathrm{P}_{I} \mathrm{O}_{2}\right)$ decreases, the isopleth is displaced to the left. The black dots correspond to the incurring alveolar gas composition at each $P_{I} O_{2}$. The line connecting all these points (thick black curve) is the alveolar air curve. $\mathrm{P}_{A} \mathrm{CO}_{2}$ and $P_{A} \mathrm{O}_{2}$ are the alveolar partial pressures of carbon dioxide and oxygen, respectively. $\dot{V}_{A} / \dot{V}_{R} C O_{2}$ alveolar ventilation to lung carbon dioxide flow ratio [Modified after Ferretti (2015)]

\section{Maintaining the equilibrium in blood: the effect of ventilation-perfusion heterogeneity}

The $\mathrm{O}_{2}-\mathrm{CO}_{2}$ diagram was established for alveolar air. Yet, a similar diagram can be constructed for blood, making use of the Fick principle. In fact, we can obtain the respiratory quotient for blood $\left(R Q_{B}\right)$ by dividing Eqs. (5a) and (5b)

$R Q_{B}=\frac{\dot{V}_{R} C O_{2}}{\dot{V}_{R} O_{2}}=\frac{\dot{Q}\left(C_{a} C_{2}-C_{\bar{v}} C O_{2}\right)}{\dot{Q}\left(C_{a} O_{2}-C_{\bar{v}} O_{2}\right)}=\frac{C_{a} C O_{2}-C_{\bar{v}} C O_{2}}{C_{a} O_{2}-C_{\bar{v}} O_{2}}$.

Of course, at steady state, $R Q_{B}=R Q_{L}=R Q_{M}$. Solving Eq. (12) for $\mathrm{C}_{a} \mathrm{CO}_{2}$, we obtain

$\mathrm{C}_{a} \mathrm{CO}_{2}=\mathrm{C}_{\bar{v}} \mathrm{CO}_{2}+\mathrm{RQ}_{B}\left(\mathrm{C}_{a} \mathrm{O}_{2}-\mathrm{C}_{\bar{v}} \mathrm{O}_{2}\right)$.

Equation (13), which, in analogy to the alveolar air equation, we may call the arterial blood equation, tells that, if we plot $\mathrm{C}_{a} \mathrm{CO}_{2}$ as a function of $\mathrm{C}_{a} \mathrm{O}_{2}$, we obtain a family of straight lines, with negative slopes equal to $R Q_{B}$, converging on a point, the coordinates of which correspond to the gas composition of mixed venous blood (Fig. 5A). This occurs in case of shunt, id est when mixed venous blood is not exposed to gas exchange in the alveolar capillaries, so that $\mathrm{C}_{a} \mathrm{O}_{2}=\mathrm{C}_{\bar{v}} \mathrm{O}_{2}$ and $\mathrm{C}_{a} \mathrm{CO}_{2}=\mathrm{C}_{\bar{v}} \mathrm{CO}_{2}$ : in this case, arterial and mixed venous bloods have the same respiratory gas composition.

Respiratory gases diffuse across the alveolar-capillary barrier, driven by differences in partial pressure. Therefore, 
A

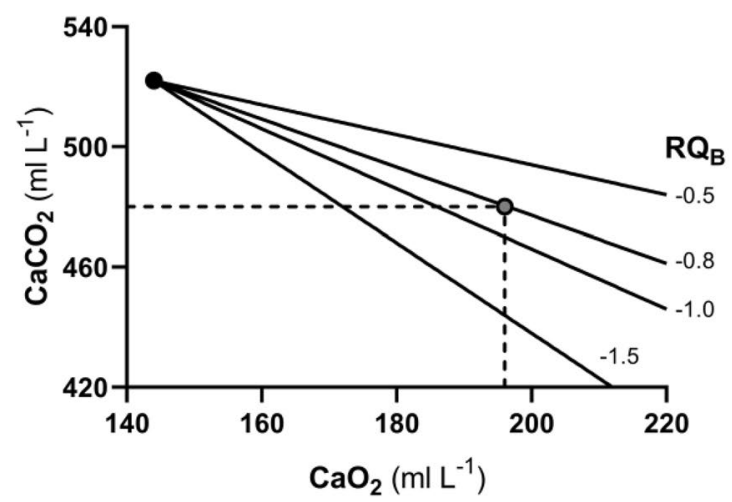

Fig. 5 A An $\mathrm{O}_{2}-\mathrm{CO}_{2}$ diagram for blood at rest. The black dot represents the mixed venous blood gas composition. Lines representing isopleths of blood respiratory quotient $\left(R Q_{B}\right)$ converge on this point. Four of these $R Q_{B}$ isopleths are reproduced (black lines). B Same as panel A, after transformation of concentrations into partial pressures. The same four isopleths of $R Q_{B}$ are reported. The black dot, on which

it may be useful to express Eq. (13) in terms of partial pressures instead of concentration (Ferretti et al. 2017)

$\beta c P_{a} \mathrm{CO}_{2}=\beta c P_{\bar{v}} \mathrm{CO}_{2}+R Q_{B} \beta o\left(P_{a} \mathrm{O}_{2}-P_{\bar{v}} \mathrm{O}_{2}\right)$,

where constants $\beta c$ and $\beta o$ are the blood transport coefficients of carbon dioxide and oxygen, respectively. On one side, $\beta c$ can be considered, on first approximation, invariant, because most of blood carbon dioxide is dissolved in plasma, either in pure form or as bicarbonate, whereas the carbon dioxide bound to haemoglobin stays on a segment of the carbon dioxide equilibrium curve that is practically linear. On the other side, contrary to $\beta c, \beta o$ cannot be taken as invariant: almost all oxygen in blood, at physiological $\mathrm{PO}_{2}$ values at sea level, is bound to haemoglobin, and, due to the shape of the oxygen equilibrium curve, $\beta o$ is lower the higher is the $\mathrm{PO}_{2}$. Thus, an a-priori model of the oxygen equilibrium curve is a prerequisite for an analytical solution of Eq. (14).

Many inductive empirical models, often polynomial, were proposed, providing detailed descriptions of the oxygen equilibrium curve (see e.g. Adair 1925; Kelman 1966; Margaria 1963; Pauling 1935; Severinghaus 1979; Tien and Gabel 1977). None of them have distinct merits over the others (Myers et al. 1990; O'Riordan et al. 1985).

Conversely, Hill (1910) adopted a theoretical approach, whose quantitative outcomes, however, were confuted when Hill's prediction of a stoichiometric oxygen/haemoglobin ratio of 2.8 was demonstrated not to correspond to the observation that indeed four molecules of oxygen can bind to each molecule of haemoglobin (Perutz 1970). Notwithstanding, although a predicted value was demonstrated to be incorrect $(n=2.8$ instead of 4 ), the deep meaning of Hill's constant was not undermined: $n>1$ indicates cooperativity; $n=1$ absence of cooperativity.
B

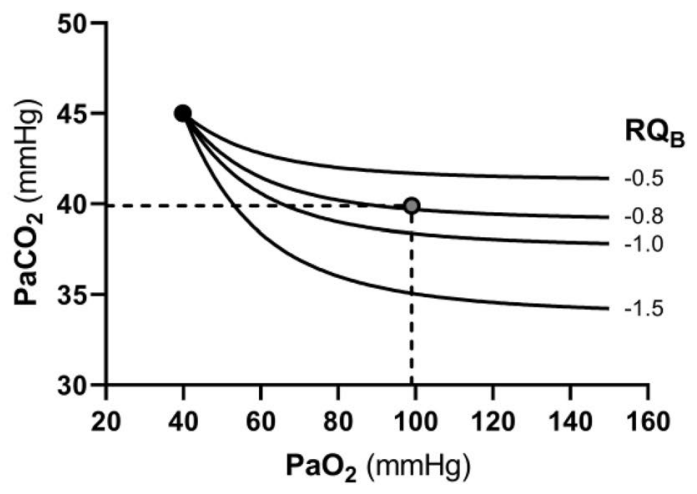

the $R Q_{B}$ isopleths converge, represents the mixed venous blood gas composition. The arterial blood gas composition of a resting human is also shown (grey dot). $\mathrm{C}_{a} \mathrm{CO}_{2}$ carbon dioxide concentration in arterial blood, $\mathrm{C}_{a} \mathrm{O}_{2}$ oxygen concentration in arterial blood, $\mathrm{P}_{a} \mathrm{CO}_{2}$ carbon dioxide partial pressure in arterial blood, $\mathrm{P}_{a} \mathrm{O}_{2}$ oxygen partial pressure in arterial blood [After Ferretti et al. (2017)]

That said, Hill's model, which provides an accurate and precise description of the oxygen equilibrium curve in the saturation range 0.20-0.98 (Severinghaus 1979), is still a fascinating tool for the analysis of the oxygen equilibrium curve, inasmuch as it is simple-two constants suffice to describe the behaviour of the curve: the slope $(n)$ and the $x$-axis intercept of Hill's plot, and because the latter constant, not yet refuted, is the basis for calculating the oxygen partial pressure sustaining a saturation of $0.50\left(P_{50}\right)$. Hill's $P_{50}$ has become a universally used parameter for the analysis of the Bohr effect, also by those who reject Hill's model.

Application of Hill's model to an analytical solution of Eq. (14) leads to the construction of the curves reported in Fig. 5B (Rahn and Fenn 1955). In this figure, four isopleths of $R Q_{B}$ are shown. Their shape is dictated by the analytical solution given to the oxygen equilibrium curve, in particular by the value taken by $\beta o$ at any $P O_{2}$. As for Fig. $5 \mathrm{~A}$, all these isopleths converge on a gas composition corresponding to that of mixed venous blood.

At exercise, the $R Q_{M}$ increases as a function of the applied power, because the fraction of energy derived from glucose oxidation through the glycolytic pathway becomes progressively larger. At about $60 \%$ of the maximal aerobic power, corresponding approximately to the so-called lactate threshold, at steady state, almost all energy is derived from carbohydrates, so that $R Q_{M}=-1.0$, and so is also $R Q_{B}$. In this condition (upper limit of the moderate exercise domain), we can assume that no net lactate accumulation occurs, so that arterial blood $\mathrm{pH}$ and $\mathrm{P}_{a} \mathrm{CO}_{2}$ remain the same as at rest. If this is so, also $\mathrm{C}_{a} \mathrm{CO}_{2}$ would remain unchanged, and thus the arterial blood gas composition. This allows the construction of Fig. 6, which evidences, on the $\mathrm{O}_{2}-\mathrm{CO}_{2}$ diagram for blood, how the blood 


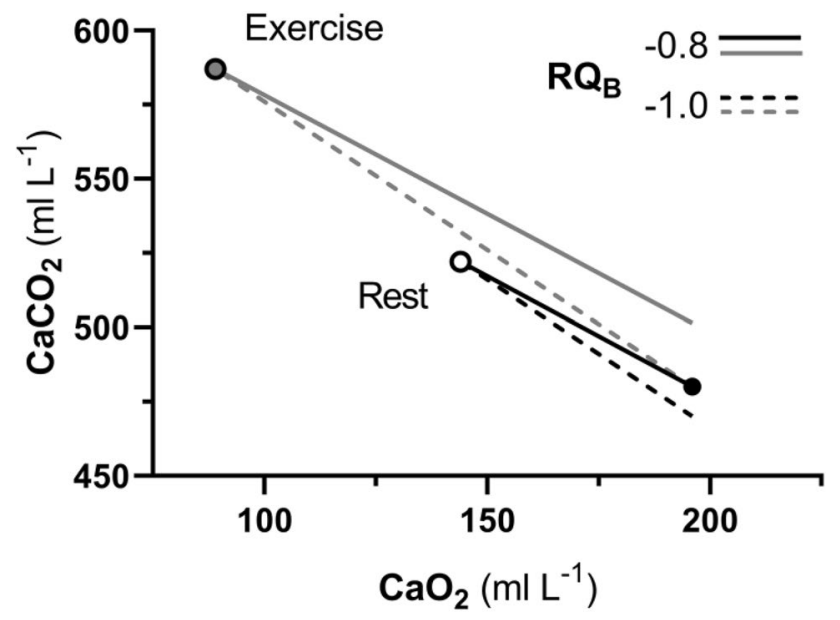

Fig. 6 An oxygen-carbon dioxide diagram for blood, on which two steady-state conditions are represented: rest and moderate exercise. The resting values are the same as in Fig. 5A: the arterial blood composition is described by the black dot, the mixed venous blood composition by the white dot. Both dots are on the $0.8 R Q_{B}$ isopleth. The black dot represents the mixed venous blood gas composition. At rest (oxygen consumption, $\dot{V} O_{2}=0.3 \mathrm{~L} \mathrm{~min}^{-1}$; blood respiratory quotient, $R Q_{B}=-0.8$; and thus carbon dioxide production $\left(\dot{V} \mathrm{CO}_{2}\right)$ is equal to $-0.24 \mathrm{~L} \mathrm{~min}^{-1}$ ), for arterial blood, we assumed: a blood haemoglobin concentration of $150 \mathrm{~g} \mathrm{~L}^{-1}$; an arterial oxygen saturation $\left(\mathrm{S}_{a} \mathrm{O}_{2}\right)$ of $0.97 ; \mathrm{pH}=7.4$; a bicarbonate concentration of $25 \mathrm{mmol} \mathrm{L}^{-1}$ (standard steady-state acid base condition, see above). The isopleth for $R Q_{B}=1.0$ is also reported. At moderate exercise, we assumed that no lactate accumulation takes place, so that arterial blood bicarbonate concentration and $\mathrm{pH}$ remain the same as at rest. We also assumed $\dot{V} O_{2}=1.0 \mathrm{~L} \mathrm{~min}{ }^{-1}, R Q_{B}=1.0$ and, being at steady state, equal to the metabolic respiratory quotient (glucose only energy source), and cardiac output as from Cerretelli and di Prampero (1987): $9 \mathrm{~L} \mathrm{~min}^{-1}$. The mixed venous oxygen concentration was then calculated using the Fick equation (Eq. 5b); the mixed venous carbon dioxide concentration was finally obtained by means of Eq. (13) (of course, since $R Q_{B}=-1.0, \dot{V} C O_{2}=-\dot{V} O_{2}=-1.0 \mathrm{~L} \mathrm{~min}^{-1}$ )

gas composition is modified during exercise. The most striking feature in Fig. 6 is the remarkable displacement of the mixed venous gas point, on which all the isopleths for $R Q_{B}$ converge. This is a direct consequence of the $\dot{V} \mathrm{O}_{2}$ and $\dot{V} \mathrm{CO}_{2}$ changes at exercise, which, since the arterial blood gas composition in the analysed condition is invariant, carry along a fall of $C_{\bar{v}} O_{2}$ and an increase of $\mathrm{C}_{\bar{v}} \mathrm{CO}_{2}$. This is the most notable difference between the $\mathrm{O}_{2}-\mathrm{CO}_{2}$ diagrams for blood and for alveolar air: in the latter case, the point on which an $R Q_{L}$ isopleth crosses the $x$-axis, corresponds to the inspired air composition, and this, at variance with the mixed venous gas composition, is independent of metabolism and of ventilation, being displaced only when we breathe a different gas mixture from air at sea level.

During steady-state rest and moderate exercise, an equilibrium is attained at the venous end of the pulmonary capillaries between end-capillary and alveolar partial pressures of gases. Therefore, if no other factors interfere with this process, arterial blood and alveolar air should have the same partial pressures of oxygen and carbon dioxide, those compatible with the same respiratory quotient in alveolar air as in blood (concepts of ideal air and ideal blood, as formulated by Rahn and Fenn 1955). At rest, when $R Q_{B}=R Q_{L}$ is generally approximated to -0.8 , these conditions are met when $P_{a} O_{2}=P_{A} O_{2}=105 \mathrm{mmHg}$ and at $P_{a} \mathrm{CO}_{2}=P_{A} \mathrm{CO}_{2}=$ $40 \mathrm{mmHg}$. A century of blood gas and alveolar gas measurements shows that the latter is the case, the former is not: despite $P_{A} O_{2}=105 \mathrm{mmHg}$ indeed, $P_{a} O_{2}=100 \mathrm{mmHg}$, so that a positive alveolar-arterial oxygen gradient appears.

Notwithstanding a small contribution from the addition of deoxygenated blood into the systemic arterial circulation from bronchial and Thebesian veins, the occurrence of a positive alveolar-arterial oxygen gradient is essentially the result of the interaction of two phenomena. The first is the heterogeneous distribution of the ventilation/perfusion ratio $\left(\dot{V}_{A} / \dot{Q}\right)$, which is not simply due to a gravitational effect (Prisk et al. 1995). $\dot{V}_{A} / \dot{Q}$ can vary between two extremes: the lowermost extreme is represented by $\dot{V}_{A} / \dot{Q}=0$ (perfused, but non ventilated lung units), in which case $P_{A} O_{2}=$ $P_{\bar{v}} O_{2}$; the uppermost extreme is represented by $\dot{V}_{A} / \dot{Q}=$ $\infty$ (ventilated, but non perfused lung units), in which case $P_{A} O_{2}=P_{I} O_{2}$ (Farhi and Rahn 1955). The second phenomenon is related to the characteristics of the oxygen equilibrium curve. Lung units with low $\dot{V}_{A} / \dot{Q}$ are characterised by lower $\mathrm{P}_{A} \mathrm{O}_{2}$ and higher $\mathrm{P}_{A} \mathrm{CO}_{2}$ than lung units with high $\dot{V}_{A} / \dot{Q}$. The respiratory gas composition of arterial blood is a perfusionweighted ${ }^{5}$ average of all gas compositions provided by all open lung capillaries, exposed to different values of $\dot{V}_{A} / \dot{Q}$, and all in equilibrium with the corresponding alveoli, at least at rest and moderate exercise. Since the relationship between $\mathrm{C}_{a} \mathrm{CO}_{2}$ and $\mathrm{P}_{a} \mathrm{CO}_{2}$ is linear in the physiological range, alveoli with high $\dot{V}_{A} / \dot{Q}$ can compensate for alveoli with low $\dot{V}_{A} / \dot{Q}$, thus ensuring $P_{a} C_{2}=P_{A} C O_{2}$. This compensation cannot occur for oxygen, as long as alveoli with low $\dot{V}_{A} / \dot{Q}$ attain an equilibrium with the corresponding capillaries on or close to the steep part of the oxygen equilibrium curve, so that arterial oxygen saturation $\left(\mathrm{S}_{a} \mathrm{O}_{2}\right)$ and $\mathrm{C}_{a} \mathrm{O}_{2}$ are lowered; in contrast, alveoli with elevated $\dot{V}_{A} / \dot{Q}$ attain an equilibrium with the corresponding capillaries on the flat part of the oxygen equilibrium curve, in a part on which any increase in $\mathrm{P}_{A} \mathrm{O}_{2}$ cannot provide an increase in $\mathrm{S}_{a} \mathrm{O}_{2}$, and thus in $\mathrm{C}_{a} \mathrm{O}_{2}$. These capillaries cannot increase their $\mathrm{C}_{a} \mathrm{O}_{2}$, and thus cannot compensate for the effect of alveoli with low $\dot{V}_{A} / \dot{Q}$ : therefore, the resulting $P_{a} O_{2}$ turns out lower than the mean

\footnotetext{
5 It is important to mention that the arterial blood concentrations are weighed by perfusion to each unit, whereas the alveolar gas concentrations are weighed by the ventilation to each unit. The heterogeneity of perfusion distribution across the lungs is much more accentuated than the heterogeneity of ventilation distribution. Therefore, the number of alveolar units with low $\dot{V}_{A} / \dot{Q}$ values is by far larger than that of alveolar units with high $\dot{V}_{A} / \dot{Q}$ values, so that the former units contribute more than the latter to the formation of arterial blood.
} 


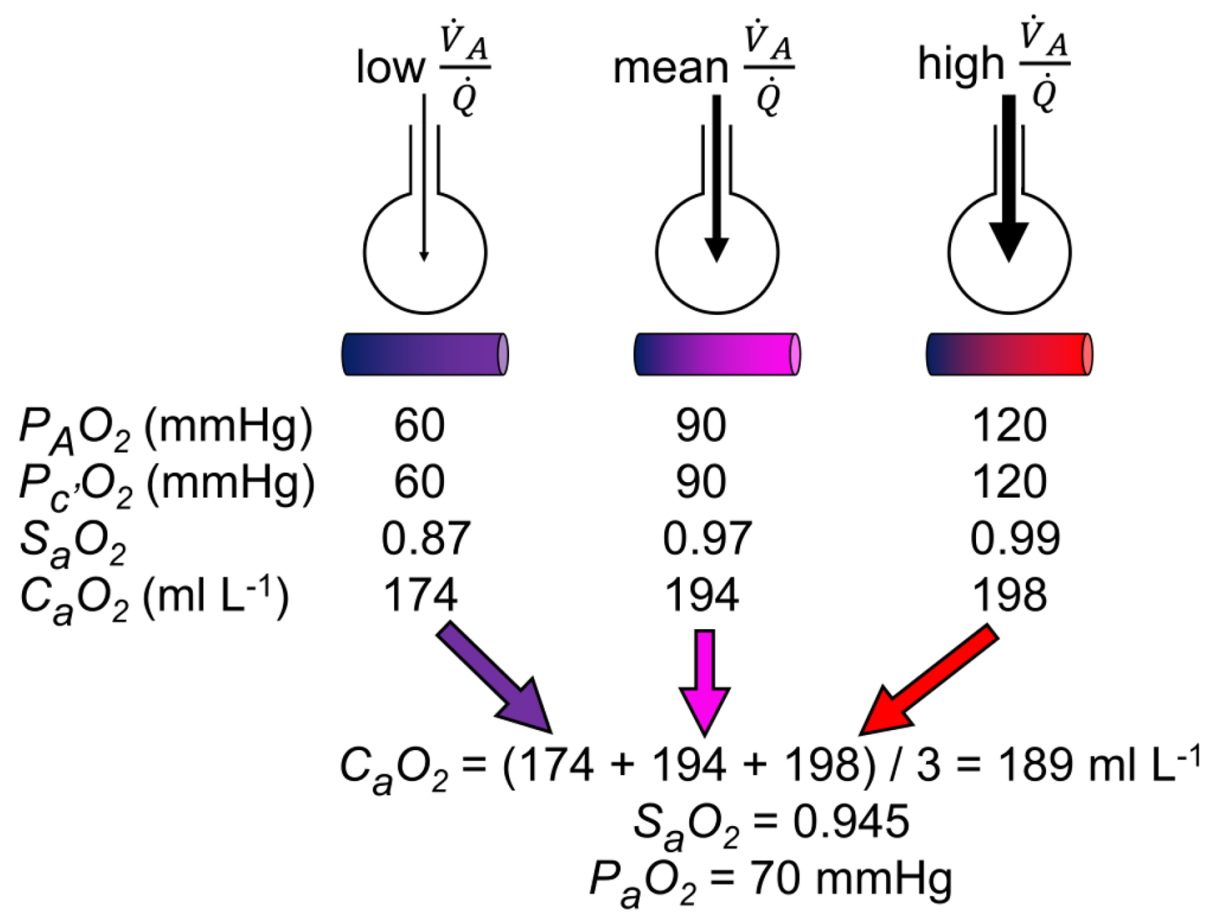

Fig. 7 Imagine a hypothetical lung with three huge lung units, each receiving the same blood flow (say $1 \mathrm{~L} \mathrm{~min}^{-1}$ for simplicity), but differently ventilated, such that, from left to right, the first is hypoventilated, the second is normoventilated, and the third is hyperventilated, thus compensating for the first. For each lung unit, the resulting alveolar $\left(\mathrm{P}_{A} \mathrm{O}_{2}\right)$ and end-capillary $\left(\mathrm{P}_{c^{\prime}} \mathrm{O}_{2}\right)$ oxygen partial pressures are reported, together with the corresponding arterial oxygen saturation $\left(\mathrm{S}_{a} \mathrm{O}_{2}\right)$ and arterial oxygen concentration $\left(\mathrm{C}_{a} \mathrm{O}_{2}\right)$. When the three units converge to form arterial blood, each contributes $1 \mathrm{~L}$ of blood

$P_{A} O_{2}$. This state of things is described with a theoretical simplified lung model in Fig. 7.

In real lungs, topographic heterogeneity of $\dot{V}_{A} / \dot{Q}$ distribution is more important when lung blood flow is low. This implies that during moderate exercise, when lung blood flow is increased, $\dot{V}_{A} / \dot{Q}$ is less heterogeneously distributed than at rest, as demonstrated using radioactive tracers (Bake et al. 1968; Bryan et al. 1964; Harf et al. 1978) or inert gases' manipulation techniques (Beck et al. 2012). Recruitment of capillaries, which are closed at rest, to sustain the increase in blood flow, may contribute significantly to this phenomenon by reducing diffusion distances between alveoli and capillaries. However, when the multiple inert gas elimination technique is used, the opposite is observed (Domino et al. 1991, 1993; Gale et al. 1985; Gledhill et al. 1978; Hammond et al. 1986). The reasons of this discrepancy during moderate exercise are yet to be understood (Wagner 1992, 2015). At variance, during sustained heavy exercise, evidence suggests that pulmonary perfusion heterogeneity is increased in humans (Burnham et al. 2009), per minute, containing, respectively, 174,194 , and $198 \mathrm{~mL}$ of oxygen. Therefore, in $1 \mathrm{~min}$, arterial blood receives $3 \mathrm{~L}$ of blood containing a total of $567 \mathrm{~mL}$ of oxygen, yielding a $C_{a} O_{2}$ of $189 \mathrm{~mL} \mathrm{~L}^{-1}$. Despite a mean $P_{A} O_{2}$ of $90 \mathrm{mmHg}$, the resulting $P_{a} O_{2}$ is $70 \mathrm{mmHg}$ only. The hyperventilated lung unit cannot compensate for the hypoventilated lung unit, and an alveolar-arterial oxygen gradient of $20 \mathrm{mmHg}$ is generated. $\dot{V}_{A}$ alveolar ventilation, $\dot{Q}$ lung capillary blood flow

suggesting the possibility that interstitial pulmonary edema may develop in this condition, thus explaining both spatial perfusion heterogeneity and $\dot{V}_{A} / \dot{Q}$ heterogeneity.

\section{The ventilation-perfusion equation}

Let us now return to Eq. (8) and solve it for $\dot{V}_{A}$

$\dot{V}_{A}=-\frac{\dot{V}_{R} C O_{2}}{F_{A} C O_{2}}$.

If we introduce a correction factor accounting for the fact that $\dot{V}_{A}$ is expressed in BTPS and $\dot{V} \mathrm{CO}_{2}$ in STPD, and we transform $F_{A} \mathrm{CO}_{2}$ in $\mathrm{P}_{A} \mathrm{CO}_{2}$ by means of Dalton's law, we obtain

$\dot{V}_{A}=-\frac{C g \dot{V}_{R} C O_{2}}{P_{A} C O_{2}}$, 
where $\mathrm{Cg}$ is the ratio of the STPD to BTPS and the $\mathrm{P}_{A} \mathrm{CO}_{2}$ to $\mathrm{F}_{\mathrm{A}} \mathrm{CO}_{2}$ conversion factors (Rahn and Fenn 1955; Otis 1964). ${ }^{6}$ Since

$\dot{V}_{R} \mathrm{CO}_{2}=R Q_{L} \dot{V}_{R} O_{2}$,

we can also write

$\dot{V}_{A}=-\frac{C g R Q_{L} \dot{V}_{R} O_{2}}{P_{A} C_{2}}$,

whence

$\dot{V}_{R} O_{2}=-\frac{\dot{V}_{A} P_{A} C O_{2}}{C g R Q_{L}}$.

Since we are at steady state, we can also write

$-\frac{\dot{V}_{A} P_{A} C O_{2}}{C g R Q_{L}}=\dot{Q}\left(C_{a} O_{2}-C_{\bar{v}} O_{2}\right)$,

whence

$-\frac{\dot{V}_{A} P_{A} C O_{2}}{C g R Q_{L} \dot{Q}\left(C_{a} O_{2}-C_{\bar{v}} O_{2}\right)}=1$.

Rearranging, we obtain

$\frac{\dot{V}_{A}}{\dot{Q}}=-\frac{C g R Q_{L}\left(C_{a} O_{2}-C_{\bar{v}} O_{2}\right)}{P_{A} C O_{2}}$.

Equation (22) is a formulation of the ventilation-perfusion equation (Rahn and Fenn 1955; Otis 1964), and sets the homeostatic equilibrium between alveolar air and blood. It states that, ceteris paribus, $\dot{V}_{A} / \dot{Q}$, which is heterogeneously distributed throughout the lungs, is directly proportional to $-R Q_{L}$ and to $\left(\mathrm{C}_{a} \mathrm{O}_{2}-\mathrm{C}_{\bar{v}} \mathrm{O}_{2}\right)$ and inversely proportional to $P_{A} C O_{2}$. Therefore, each $P_{A} O_{2}$ value in Fig. 3 corresponds not only to one $R Q_{L}$ value, but also to a unique value of $\dot{V}_{A} / \dot{Q}$. This value is lower the closer $R Q_{L}$ is to zero.

Equation (22) applies to specific values of $\dot{V}_{A}$ and $\dot{Q}$, and thus to given metabolic levels. Since, within the aerobic exercise domain at sea level, as long as $\mathrm{P}_{\mathrm{A}} \mathrm{CO}_{2}$ stays invariant and equal to $40 \mathrm{mmHg}$, also $\dot{V}_{A} / \dot{V}_{R} \mathrm{CO}_{2}$ stays invariant and equal to -21.6 , we can derive a more general equation by combining Eqs. (5b) and (22) and rearranging, as follows:

$$
\begin{aligned}
\frac{\dot{V}_{A}}{\dot{V}_{R} C O_{2}}= & \frac{\dot{V}_{A}}{\dot{Q}\left(C_{a} C O_{2}-C_{\bar{v}} C O_{2}\right)} \\
& =\frac{C g R Q_{L}\left(C_{a} O_{2}-C_{\bar{v}} O_{2}\right)}{P_{A} C O_{2}\left(C_{\bar{v}} C O_{2}-C_{a} C O_{2}\right)}=-21.6 .
\end{aligned}
$$

$\overline{{ }^{6} \text { In fact } \dot{V}_{A}}=-\frac{\frac{760}{P_{B}-47} \frac{310}{273} R Q_{L} \dot{V}_{R} O_{2}}{\frac{P_{A} C O_{2}}{P_{B}-47}}$. Since the two terms $\left(P_{B}-47\right)$ cancel out, the proportionality constant in Eq. (16) $(C g)$ turns out equal to $760 * 310 / 273$, i.e., $863 \mathrm{mmHg}$ if $\dot{V}_{R} C O_{2}$ is expressed in $\mathrm{L} \mathrm{min}{ }^{-1}$, or $0.863 \mathrm{mmHg}$ if $\dot{V}_{R} \mathrm{CO}_{2}$ is expressed in $\mathrm{ml} \mathrm{min}^{-1}$.
During exercise, as compared to rest, $\dot{V}_{A}$ increases in direct proportion to $-\dot{V} \mathrm{CO}_{2}$, whereas the increase of $\dot{Q}$ is less. Therefore, if $\dot{V}_{A} / \dot{V}_{R} C O_{2}$ stays invariant, also $\mathrm{C}_{\bar{v}} \mathrm{CO}_{2}-\mathrm{C}_{a} \mathrm{CO}_{2}$ must increase, because of higher $\mathrm{C}_{\bar{v}} \mathrm{CO}_{2}$. Moreover, the $\dot{V}_{A} / \dot{Q}$ grows, being higher the higher is the metabolic rate, inasmuch as, not only $\mathrm{C}_{\bar{v}} \mathrm{CO}_{2}-\mathrm{C}_{a} \mathrm{CO}_{2}$ goes up, but $R Q_{L}=R Q_{M}$ tends to approach -1 , due to a progressive shift toward carbohydrate oxidation. It is noteworthy, however, that, as long as we stay at sea level, and thus, we operate on the flat part of the oxygen equilibrium curve, $\mathrm{C}_{a} \mathrm{O}_{2}$ does not vary, at least at rest and during moderate exercise, so that an increase of $\left(\mathrm{C}_{a} \mathrm{O}_{2}-\mathrm{C}_{\bar{v}} \mathrm{O}_{2}\right)$ can be sustained solely by a fall of $\mathrm{C}_{\bar{v}} \mathrm{O}_{2}$. By analogy, as long as $\mathrm{P}_{a} \mathrm{CO}_{2}$ stays invariant, and so does $\mathrm{C}_{a} \mathrm{CO}_{2}$, a decrease of $\left(\mathrm{C}_{a} \mathrm{CO}_{2}-\mathrm{C}_{\bar{v}} \mathrm{CO}_{2}\right)$ can be sustained solely by an increase in $\mathrm{C}_{\bar{v}} \mathrm{CO}_{2}$.

The tight matching between $\dot{V}_{A}$ and $-\dot{V} C O_{2}$ reflects a fine regulation, mostly centred on the modulation of ventilation by the activity of central chemoreceptors. This equilibrium is broken in case of hyperventilation: the $\dot{V}_{A} / \dot{V}_{R} C O_{2}$ goes down (becomes more negative), a new steady state is attained at a lower $P_{a} \mathrm{CO}_{2}$, and at a higher $P_{a} O_{2}$, than those incurring during normoventilation $\left(\mathrm{P}_{a} \mathrm{CO}_{2}=40 \mathrm{mmHg}\right)$ at any given $P_{I} \mathrm{O}_{2}$. This occurs, e.g., at high altitude, because of hypoxic stimulation of peripheral chemoreceptors, or in case of a larger $\dot{V}_{A} / \dot{Q}$ heterogeneity in the lungs, due to the presence of either non ventilated lung regions or increased physiological dead space (unperfused lung regions). The equilibrium is broken in the opposite direction in case of hypoventilation, as in respiratory failure or paralysis of the respiratory centres.

\section{Diffusion-perfusion interaction in alveolar- capillary gas transfer}

The homeostatic equilibria discussed so far, leading to a tight coupling of respiration to metabolism, rely on the implicit assumption of complete gas equilibration between alveolar air and capillary blood. In normoxic humans at rest and at steady-state exercise up to the critical power, provided the alveolar-capillary barrier be intact, such equilibration may occur indeed in each open pulmonary capillary in contact with an alveolus, independent of its $\dot{V}_{A} / \dot{Q}$, inasmuch as the contact time between alveolar air and capillary blood is long enough. According to Wagner and West (1972), at rest, full equilibration between the two sides of the alveolar-capillary barrier is attained when the blood has completed about one-third of the capillary length, a distance that Heller and Schuster (2007) have reduced to one-seventh. This provides a remarkable 
reserve, to be exploited during exercise, for alveolar-capillary gas equilibration. The same authors have suggested, based on the data of Borland et al. (2001), that this is the case also in the heavy exercise domain, thanks to this reserve. The report by Hakim et al. (1994) that a four-time increase in pulmonary blood flow is accompanied by a reduction by one half of the capillary transit time suggests alveolar-capillary equilibration over a large spectrum of exercise intensities. In non-athletic subjects, the extreme limits of this functional equilibrium may be attained at maximal exercise (Heller and Schuster 2007).

In athletic subjects, with elevated maximal $\dot{V} \mathrm{O}_{2}$ and maximal $\dot{Q}$, no alveolar-capillary gas equilibration is attained at maximal exercise, incidentally an unsteadystate condition. Thus, the phenomenon of exercise-induced arterial hypoxaemia, which was reported for the first time by Harrop (1919), appears around maximal exercise (Dempsey et al. 1984; Dempsey and Wagner 1999), and eventually even in submaximal exercise, especially in women (Dominelli et al. 2013; Harms et al. 1998). Besides diffusion limitation in alveolar-capillary oxygen transfer, which we discuss here below, insufficient hyperventilation due to expiratory flow limitation, and increased $\dot{V}_{A} / \dot{Q}$ heterogeneity at maximal exercise have also been called upon as possible mechanisms behind exercise-induced arterial hypoxaemia (Dempsey and Wagner 1999; Nielsen 2003; Prefaut et al. 2000). Some functional consequences of exercise-induced arterial hypoxaemia on oxygen flow during maximal exercise have been discussed elsewhere (Ferretti 2014). Incidentally, it is noteworthy that full equilibration in alveolar-capillary gas exchange is still compatible with the occurrence of a positive alveolar-arterial oxygen gradient, the nature of which, as discussed above, is of different origin.

The hypothesis of diffusion limitation to explain exerciseinduced arterial hypoxaemia stems from Piiper's model of alveolar gas exchange (Piiper and Scheid 1981), characterised by the diffusion-perfusion interaction equations. The model's axiom is that alveolar-capillary gas transfer results from two interacting mechanisms: diffusion across the alveolar-capillary barrier, and lung capillary perfusion. At steady state, the flow of any gas removed from or added to the lungs through the airways is equal to the flow of gas crossing the alveolar-capillary barrier. A simple model of alveolar-capillary gas exchange can be constructed by imagining the mean pulmonary blood flow in contact with the mean alveolar air, with oxygen diffusing across a thin membrane separating capillary blood from alveolar air (see Fig. 8). In such a system, the gas flow $\dot{V}$ across the membrane of given surface area $A$ and thickness $L$ is directly proportional to the pressure gradient across that membrane

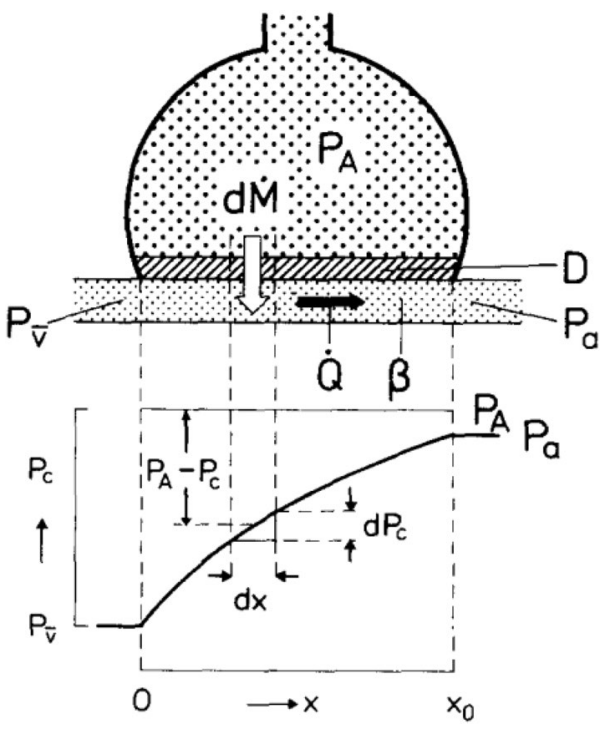

Fig. 8 A scheme of the simplified model of the lung. The model implies numerous simplifying assumptions: steady state, diffusion occurring across a flat homogeneous alveolar-capillary barrier, and along a direction $D$ that is perpendicular to the barrier; linear blood dissociation curve (constant gas transport coefficient for blood, $\beta$ ); homogeneous membrane and blood flow $(\dot{Q})$; absence of shunt. The graph describes the exponential increase of gas partial pressure inside the capillary, as blood proceeds along it. The flow across the barrier follows the pressure gradient. $d \dot{M}$ infinitesimal molar flow of the gas, $P_{A}$ alveolar gas pressure, $P \bar{v}$ mixed venous gas pressure, $P_{a}$ arterial gas pressure, $P_{c}$ capillary gas pressure, $x$ distance along the capillary, $O$ beginning of pulmonary capillary, $X_{0}$ end of capillary, where $P_{c^{\prime}} O_{2}$ is attained [From Piiper and Scheid (1981)]

$\dot{V}=\Delta P\left(\frac{d s A}{L}\right)=\Delta P D_{L}$,

where $d$ and $s$ are the diffusion and solubility constants in the barrier of the gas at stake, and $D_{L}$ is a lumped constant, which we call lung diffusing capacity (Bates et al. 1955; Wagner 1977). $\Delta P$ is the effective pressure gradient, id est the difference between $P_{A} O_{2}$ and the pressure existing in the capillary at a given distance $\delta$ from the venous entrance $\left[P_{c}(\delta)\right]$.

Restricting the analysis to oxygen, the diffusive oxygen flow $\left(\dot{V}_{L} O_{2}\right)$ across the barrier at any distance $\delta$ turns out equal to

$d \dot{V}_{L} O_{2}=\left[P_{A} O_{2}-P_{c} O_{2}(\delta)\right] D_{L}$,

where $P_{c} O_{2}(\delta)$ is the oxygen partial pressure in a lung capillary at distance $\delta$ from the arterial pulmonary entrance (venous blood). The infinitesimal $d \dot{V} O_{2}$ across the barrier raises $P_{c} O_{2}(\delta)$ as we proceed along the capillary from the venous to the arterial side.

The rate of increase of $P_{c} O_{2}(\delta)$ is inversely proportional to the lung capillary blood flow (set equal to $\dot{Q}$ in absence 
of shunt) and to the blood transport coefficient $\beta$ of the gas at stake (for oxygen, $\beta o$ )

$d \dot{V}_{L} O_{2}=\dot{Q} \beta o d P_{c} O_{2}$.

For inert gases, $\beta$ is a constant that is independent of the gas pressure. For oxygen, $\beta o$ depends on $\mathrm{P}_{c} \mathrm{O}_{2}$ because of the characteristics of the oxygen equilibrium curve. If for simplification, we assume $\beta o$ invariant (this assumption is discussed below), the $P_{c} O_{2}$ along the capillary tends to an asymptote corresponding to $P_{A} O_{2}$ (Krogh 1922). On this basis, at steady state, combination of Eqs. (25) and (26), which provide equal values of $d \dot{V}_{L} O_{2}$, and subsequent integration along the capillary length, yields (Piiper and Scheid 1981)

$\frac{P_{A} O_{2}-P_{c^{\prime}} O_{2}}{P_{A} O_{2}-P_{\bar{v}} O_{2}}=e^{-\frac{D_{L}}{Q \beta \beta o}}$,

where $\mathrm{P}_{c^{\prime}} \mathrm{O}_{2}$ is end-capillary $\mathrm{PO}_{2}$. This equation is called the lung diffusion-perfusion interaction equation. Piiper and Scheid (1981) named the left-hand side of Eq. (27) equilibration deficit. Its value depends only on the equilibration coefficient $\left(K_{e}\right)$, defined as the module of the exponent of the right-hand side of Eq. (27). In fact, $K_{e}$ is the ratio of two conductances in series, namely the diffusive conductance across the barrier $\left(D_{L}\right)$ and the perfusive conductance $(\dot{Q} \beta o)$ (Piiper and Scheid 1981), and is therefore dimensionless.

The larger is $K_{e}$, the closer to zero is the equilibration deficit. Therefore, when $K_{e}$ is large (>3), the equilibration deficit approaches 0 , so that $P_{c^{\prime}}$ is practically equal to $P_{A}$, alveolus and capillary have attained equilibrium and the gas flow is limited by perfusion. On the other side, when $K_{e}$ is sufficiently small $(<0.1)$, the equilibration deficit gets close to $1, P_{c^{\prime}}$ approaches $P_{\bar{v}}$, and the gas flow is limited by diffusion.

For oxygen, most of which is carried by haemoglobin, diffusion limitation occurs when we operate on the steep part of the oxygen equilibrium curve (high $\beta o$ ), as in deep hypoxia at altitude or in case of enlarged $\frac{V_{A}}{\dot{Q}}$ heterogeneity. This also occurs in endurance athletes, who undergo exercise-induced arterial hypoxaemia. On the contrary, perfusion limitation occurs when we operate on the flat part of the oxygen equilibrium curve (low $\beta o$ ), as in normoxia or in hyperoxia. The flow of carbon monoxide, which has an extremely high affinity for haemoglobin (very high $\beta$ ), is limited by diffusion. For all inert gases, which do not bind to haemoglobin, and thus are subject only to the law of Henry, gas flow is limited by perfusion. In this case, $K_{e}$ is roughly inversely proportional to the gas molecular weight (Kawashiro et al. 1975). Generally speaking, there is perfusion limitation if $\beta$ is high; there is diffusion limitation when $\beta$ tends to 0 . Differences in $K_{e}$ among gases depend only on their diffusion and solubility constants (thus on $D_{L}$ ), and on $\beta$ as long as $K_{e}$ is proportional to the ratio $d s / \beta$. This general principle is crucial in determining the size of the alveolar-arterial oxygen gradient.

We define contact time $\left(t_{c}\right)$ the time taken by blood to proceed along a capillary from one side to the other. It is equal to the ratio of the effective lung capillary blood volume $\left(q_{c}\right)$ to $\dot{Q}$. So $K_{e}$ can be expressed as follows:

$K_{e}=\frac{D_{L} t_{c}}{q_{c} \beta o}$.

At rest, $q_{c}$ is assumed invariant and independent of $\dot{Q}$. At exercise, the increase of $\dot{Q}$ carries along not only an increase in $q_{c}$, due to the recruitment of previously closed capillaries, but also a fall of $t_{c}$, because the increase in $\dot{Q}$ overrides that in $q_{c}$. Therefore, $K_{e}$ is lowered at exercise, and the equilibration deficit is higher. This tendency enhances the probability of observing diffusion limitation at exercise with respect to rest. Diffusion limitation is a major determinant of the increase in the alveolar-arterial oxygen difference during heavy exercise in individuals characterised by very high levels of maximal $\dot{V} O_{2}$ in normoxia, or in normal people in hypoxia (Wagner 2015).

This model of the alveolar-capillary gas transfer assumes not only steady state, but also constancy of $\beta$ and homogeneity of the alveolar-capillary barrier. The former assumption is ensured for inert gases which do not bind to transport proteins: they are merely dissolved in plasma and the Henry's law applies to them. It can also be assumed for carbon dioxide, for its dissociation curve can be approximated to a straight line in the physiological partial pressure range. It cannot be assumed for oxygen, due to the allosteric characteristics of the oxygen equilibrium curve. A simplification making this assumption plausible is to take the mean slope of the oxygen equilibrium curve $(\overline{\beta o})$, which is equal to

$\overline{\beta o}=\frac{C_{a} O_{2}-C_{\bar{v}} O_{2}}{P_{a} O_{2}-P_{\bar{v}} O_{2}}$.

This implies that $\overline{\beta o}$ is higher the closer the $P_{a} O_{2}$ is to the steep part of the oxygen equilibrium curve, thus being higher in hypoxia than in normoxia, coherently with the notion, expressed above, that $K_{e}$ is low in hypoxia (diffusion limitation) and high in normoxia (perfusion limitation).

The second assumption, that of a homogeneous alveolar-capillary barrier, is false both structurally, as demonstrated by morphometric analysis of the lungs (Constantinopol et al. 1989; Weibel 1972, 1999), and functionally, as long as lung capillary perfusion is heterogeneously distributed both in space and in time (Glenny and Robertson 1990, 2011; Hlastala et al. 1996). The simplified model of the lung used by Piiper and Scheid (1981) circumvents this problem by admitting an average alveolar-capillary barrier. 


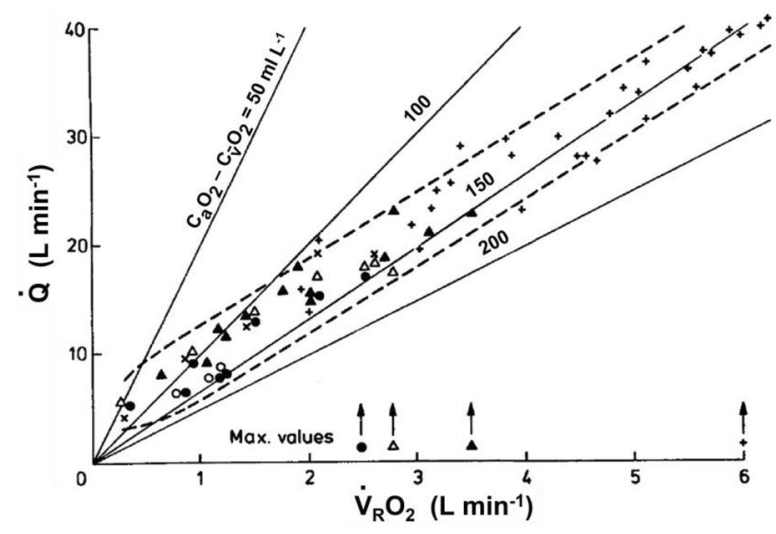

Fig. 9 Cardiac output $(\dot{Q})$ as a function of oxygen flow $\left(\dot{V} \mathrm{O}_{2}\right.$ in the figure, $\dot{V}_{R} O_{2}$ in the text) for various groups of subjects of different maximal oxygen consumption. Each symbol designates a group of subjects. Isopleths showing arterial-mixed-venous oxygen difference in $\mathrm{ml}$ of oxygen per $\mathrm{L}$ of blood $\left(\mathrm{C}_{a} \mathrm{O}_{2}-\mathrm{C}_{\bar{v}} \mathrm{O}_{2}\right.$ in the text) are also drawn [Modified after Cerretelli and di Prampero (1987)]

The effects of a heterogeneous distribution of $D_{L}$ through the lungs on the alveolar-capillary gas transfer were analysed by Piiper (1992).

Similar models were used to analyse gas transfer in peripheral systemic capillaries (Piiper et al. 1984; Piiper and Scheid 1999), after accounting for the fact that oxygen is consumed in the cells, which has an impact on intracellular oxygen gradients. These models are variants of the classical Krogh's cylinder model (Krogh 1919), in which the entire diffusion resistance is confined into the boundary between tissue and blood (Piiper et al. 1984). For a deep and thorough discussion of the subject of peripheral gas exchange, we refer the readers to the beautiful review by David Poole and coworkers in this series (Poole et al. 2022).

\section{On the cardiovascular responses to exercise}

Coupling of respiration to metabolism in blood during exercise is largely ensured by the increase in $\dot{Q}$ (Asmussen and Nielsen 1952; Åstrand et al. 1964; Hermansen et al. 1970; Stenberg et al. 1967). At steady state, $\dot{Q}$ has been treated as a linear function of $\dot{V}_{R} O_{2}{ }^{7}$ (Åstrand et al. 1964; Cerretelli and di Prampero 1987), but it is not directly proportional to $\dot{V}_{R} O_{2}$. The relation has a positive $y$-intercept, implying that $\dot{Q}$ at exercise is not proportional to $\dot{V}_{R} O_{2}$ : in fact at maximal exercise, in young non-athletic subjects, $\dot{Q}$ is only four times higher than at rest, whereas $\dot{V}_{R} O_{2}$ is about ten times higher than at rest. Therefore, according to Eq. (5a), the increase

\footnotetext{
7 Throughout the text, the acronym $\dot{V}_{R} O_{2}$ is used for oxygen flow, because it is the oxygen flow along the respiratory system. However, since these are steady-state relationships, wherein $\dot{V}_{R} O_{2}=\dot{V} O_{2}$, we could also use $\dot{V} O_{2}$ instead, as Cerretelli and di Prampero (1987) did.
}

of $\dot{V}_{R} O_{2}$ at exercise and its equality with $\dot{V} O_{2}$ is made possible only by the simultaneous increase of $\mathrm{C}_{a} \mathrm{O}_{2}-\mathrm{C}_{\bar{v}} \mathrm{O}_{2}$. This is clearly shown in Fig. 9. Therefore, two variables determine the tight matching of $\dot{V}_{R} O_{2}$ with $\dot{V} O_{2}$ at exercise: $\dot{Q}$ and $\mathrm{C}_{a} \mathrm{O}_{2}-\mathrm{C}_{\bar{v}} \mathrm{O}_{2}$.

The increase in $\dot{Q}$ results from the simultaneous increase of its two determinants, $f_{H}$ and the stroke volume $\left(Q_{s}\right)$. This increase is classically attributed to a re-modulation of autonomic control of heart activity at exercise (Fagraeus and Linnarsson 1976; Robinson et al. 1966). The increase of $Q_{s}$ is also attributed to the effect of the Frank-Starling mechanism (Rowell et al. 1996). During steady-state dynamic exercise, $f_{H}$ increases linearly with $\dot{V}_{R} O_{2}$. The increase of $Q_{s}$ as a function of $\dot{V}_{R} O_{2}$ was not treated mathematically, but Åstrand et al. (1964) considered it as non-linear. If the latter were the case, it would not be compatible with a linear $\dot{Q}$ versus $\dot{V}_{R} O_{2}$ relationship.

At rest, the largest fraction of $\dot{Q}$ goes to the kidneys. At exercise, most of the increase in $\dot{Q}$ goes into the active muscle mass. Muscle blood flow increases dramatically, due to the stimulation of $\beta_{2}$-adrenergic receptors, which are present in muscle arterioles instead of $\alpha_{1}$-adrenergic receptors, and to the action of local vasodilating mediators that are liberated as muscles contract (Casey and Joyner 2011; Delp and Laughlin 1998; Delp and O'Leary 2004; Hearon and Dinenno 2016; Mortensen and Saltin 2014; Saltin et al. 1998; Seals and Victor 1991). The activation of ATP-sensitive potassium channels by nitric monoxide plays also an important role in muscle vasodilation (Dora 2016; Schrage et al. 2006). Muscle vasodilation is almost immediate at the beginning of dynamic exercise and leads to a sudden increase in muscle blood flow (Chin et al. 2010; Clifford 2007; DeLorey et al. 2003; Ferretti et al. 1995) and to a dramatic fall of peripheral resistance $\left(R_{p}\right)$ (Elstad et al. 2009; Faisal et al. 2010; Lador et al. 2006, 2008; Wieling et al. 1996). After a transient initial decrease, systolic arterial pressure increases, whereas diastolic blood pressure, if anything, tends to go down (Rowell et al. 1968).

The reported linear $\dot{Q}$ versus $\dot{V}_{R} O_{2}$ relationship is remarkably stable in a variety of conditions, including aerobic exercise training, following which a decrease of $f_{H}$ compensates for an increase of $Q_{s}$, so that at any given $\dot{V}_{R} O_{2}, \dot{Q}$ remains unchanged (Ekblom et al. 1968; Saltin et al. 1968). The same is the case for athletes, who have the same $\dot{Q}$ versus $\dot{V}_{R} O_{2}$ relationship as non-athletes, with lower $f_{H}$ and higher $Q_{s}$ (Ekblom and Hermansen 1968). The superposition of arm exercise to leg exercise (Bevegård et al. 1966; Secher et al. 1977) and the exercise mode (Hermansen et al. 1970) do not alter the $\dot{Q}$ versus $\dot{V}_{R} O_{2}$ relationship. Water immersion and supine posture, despite their acute effects on central blood volume with consequent decrease in $f_{H}$ and increase in $Q_{s}$, leave the 
$\dot{Q}$ versus $\dot{V}_{R} O_{2}$ relationship unchanged (Bevegård et al. 1966; Leyk et al. 1994; Rennie et al. 1971; Sheldahl et al. 1987). Analogous effects are obtained with exposure to lower body negative pressure, although in some cases, heart rate compensation was incomplete (Chang et al. 1994; Eiken and Bjurstedt 1985; Fagoni et al. 2020). Exercise in the heat, whose effects on $f_{H}$ and $Q_{s}$ are opposite to those of water immersion, does not affect the $\dot{Q}$ versus $\dot{V}_{R} O_{2}$ relationship, as well. Only in extreme heat $\dot{Q}$ becomes lower at any given $\dot{V}_{R} O_{2}$, due to the extreme fall of $R_{p}$ occurring when simultaneous strong vasodilation of both the muscular and cutaneous districts intervenes in association with the reduction of plasma volume due to massive sweating (Nadel et al. 1979; Rowell 1974; Périard et al. 2021). Splanchnic and renal vasoconstriction tend to mitigate these effects (Rowell 1974). The classical view was that $\dot{Q}$ is maintained by opposite compensatory changes on $f_{H}$ and $Q_{s}$, thanks to a short-term modulation by the autonomic nervous system (Cerretelli and di Prampero 1987).

Notwithstanding, there are two conditions in which the $\dot{Q}$ versus $\dot{V}_{R} O_{2}$ relationship appears to be shifted upwards with respect to that observed at sea level: acute hypoxia (Hartley et al. 1973; Hughes et al. 1968; Roca et al. 1989; Stenberg et al. 1966) and moderate carbon monoxide poisoning (Ekblom et al. 1975; Vogel and Gleser 1972). Both these conditions lead to a reduction of $\mathrm{C}_{a} \mathrm{O}_{2}$. Conversely, when $\mathrm{C}_{a} \mathrm{O}_{2}$ is increased, such as upon return to sea level after altitude acclimatization, the $\dot{Q}$ versus $\dot{V}_{R} O_{2}$ relationship is shifted downward (Ferretti et al. 1990; Steinacker et al. 1996). Ferretti et al. (1990) hypothesized that $\dot{Q}$ is inversely proportional to $\mathrm{C}_{a} \mathrm{O}_{2}$, such that their product, equal to the systemic oxygen delivery $\left(\dot{Q}_{a} O_{2}\right)$, is kept constant at any given $\dot{V}_{R} O_{2}$. This suggests that $\dot{Q}_{a} O_{2}$ rather than $\dot{Q}$ may be the regulated variable at exercise steady state, varying at exercise as a function of $\dot{V}_{R} O_{2}$ in such a way as to ensure tight coupling between $\dot{V} O_{2}$ and $\dot{V}_{R} O_{2}$. Ferretti et al. (1992) showed that this was the case indeed, demonstrating the postulated relationship of inverse proportionality between $\dot{Q}$ and $\mathrm{C}_{a} \mathrm{O}_{2}$; this finding was later confirmed by Calbet et al. (2006) on a wider basis.

Ferretti et al. (1992) investigated also the relationship between $\dot{Q}_{a} \mathrm{O}_{2}$ and power in the moderate exercise power range. They found it to be linear and parallel to that between $\dot{V} O_{2}$ and power. Therefore, the vertical difference between the two lines, corresponding to the oxygen flow in mixed venous blood $\left(\dot{Q}_{\bar{v}} O_{2}=\dot{Q} C_{\bar{v}} O_{2}\right)$, is a constant, independent of the exercise intensity. The constant $\dot{Q}_{\bar{v}} O_{2}$ depends on $\mathrm{S}_{a} \mathrm{O}_{2}$ (Anchisi et al. 2001) and thus on $\mathrm{C}_{a} \mathrm{O}_{2}$, being lower in hypoxia than in acute normoxia.

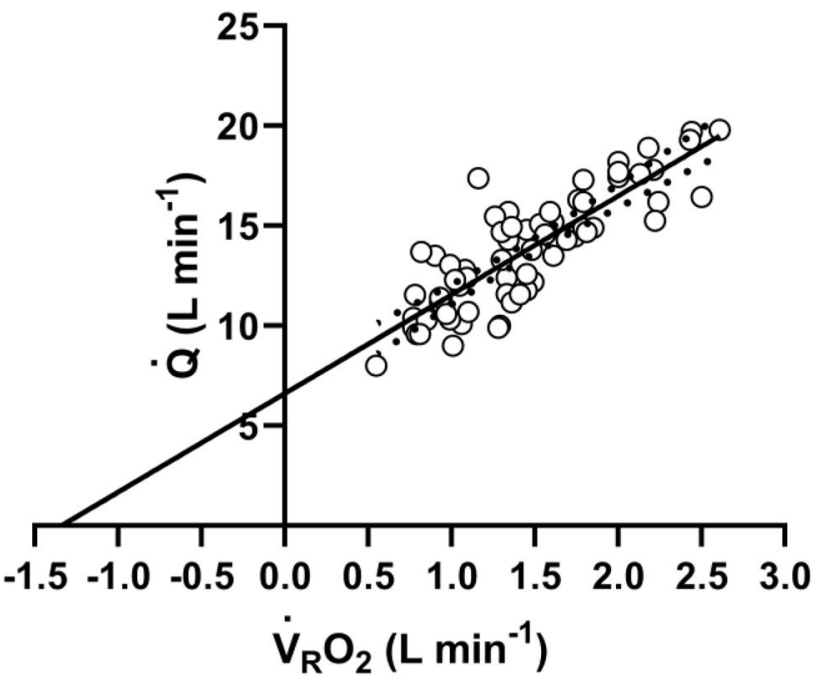

Fig. 10 Cardiac output $(\dot{Q})$ as a function of oxygen flow $\left(\dot{V}_{R} O_{2}\right)$. All reported data, from various sources in the literature, were obtained during steady-state exercise in normoxia. The regression equation yields an $x$-axis intercept of $-1.35 \mathrm{~L} \mathrm{~min}^{-1}$, and a slope equal to $4.93 \mathrm{~L}$ of blood per $\mathrm{L}$ of oxygen, whence an oxygen flow in mixed venous blood of $1.35 \mathrm{~L} \mathrm{~min}^{-1}$ and an arterial oxygen concentration of $203 \mathrm{~mL} \mathrm{~L}^{-1}$. The continuous thick line corresponds to the regression equation; the dotted lines describe the confidence intervals [Modified after Adami et al. (2014)]

\section{The $\dot{Q}-\dot{V}_{R} \mathrm{O}_{2}$ diagram}

Assuming constant $\dot{Q}_{\bar{v}} O_{2}$, Adami et al. (2014) analysed the $\dot{Q}$ versus $\dot{V}_{R} O_{2}$ relationship from a different perspective. In fact, $\dot{Q}_{\bar{v}} O_{2}$ is equal to

$\dot{Q}_{\bar{v}} O_{2}=K=\dot{Q}_{a} O_{2}-\dot{V}_{R} O_{2}=\dot{Q} C_{a} O_{2}-\dot{V}_{R} O_{2}$,

whose solution for $\dot{Q}$ is

$\dot{Q}=\frac{\dot{V}_{R} O_{2}+\dot{Q}_{\bar{v}} O_{2}}{C_{a} O_{2}}$

Equation (31) implies that, if we plot $\dot{Q}$ as a function of $\dot{V}_{R} O_{2}$, we obtain a linear relationship (Fig. 10), with slope equal to $\mathrm{C}_{a} \mathrm{O}_{2}{ }^{-1}$ and $x$-axis intercept equal to $-\dot{Q}_{\bar{v}} \mathrm{O}_{2}$. Regression through the experimental data reported in Fig. 10 yielded a mean $\dot{Q}_{\bar{v}} O_{2}$ of $1.35 \mathrm{~L} \mathrm{~min}^{-1}$, whereas $\mathrm{C}_{a} \mathrm{O}_{2}{ }^{-1}$ resulted equal to $4.93 \mathrm{~L}$ of blood per $\mathrm{L}$ of oxygen, yielding a theoretical $C_{a} O_{2}$ of $203 \mathrm{~mL} \mathrm{~L}^{-1}$. The slope of such a relationship should be higher in women than in men, due to the lower blood haemoglobin concentration in the former (Garry et al. 1954). Similarly, it should be increased in acute anaemia and decreased in acute polycythaemia.

Based on the data shown in Fig. 10, Adami et al. (2014) constructed a novel theoretical view of the steady-state cardiovascular responses to exercise as a function of the 


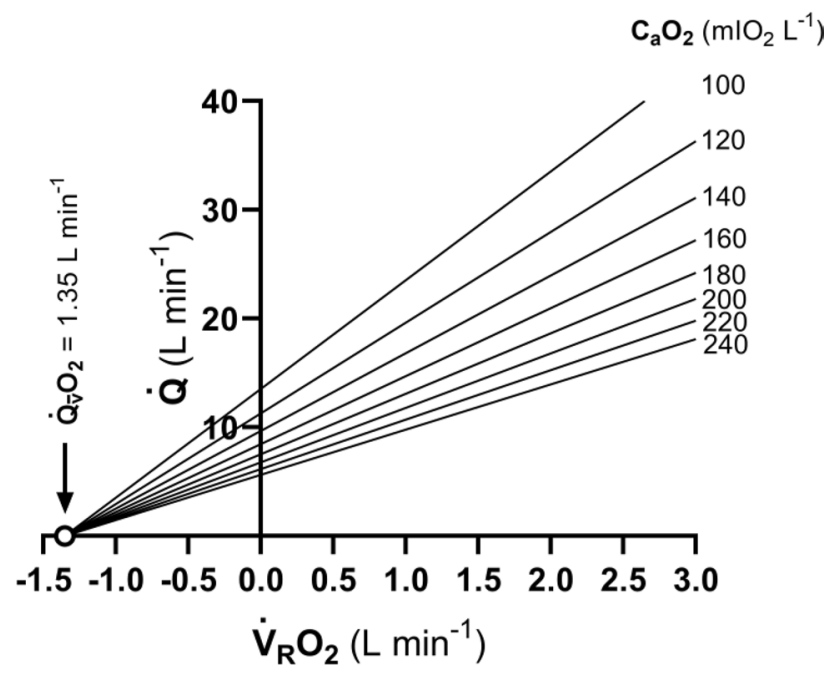

Fig. 11 Theoretical representation of the relationship between cardiac output $(\dot{Q})$ and oxygen flow $\left(\dot{V}_{R} O_{2}\right)$ in exercising humans. Continuous lines are isopleths for the arterial oxygen concentration values $\left(C_{a} O_{2}\right.$, in $\left.\mathrm{mLO}_{2} \mathrm{~L}^{-1}\right)$ indicated on top right of each line. These lines converge on the same $x$-axis intercept (open dot) that permits to compute the value of the oxygen flow in mixed venous blood $\left(\dot{Q}_{\bar{v}} O_{2}\right.$, $1.35 \mathrm{~L} \mathrm{~min}^{-1}$ )

steady-state $\dot{V} O_{2}$ and $\mathrm{C}_{a} \mathrm{O}_{2}$, which they called the $\dot{Q}-\dot{V}_{R} \mathrm{O}_{2}$ diagram. This view, expressed in graphical form in Fig. 11, is conceptually different from the classical $\dot{Q}$ versus $\dot{V}_{R} O_{2}$ relationship shown in Fig. 9. The latter figure, in fact, shows a progressive shift of the $\dot{Q}$ values toward isopleths for higher $\mathrm{C}_{a} \mathrm{O}_{2}-\mathrm{C}_{\bar{v}} \mathrm{O}_{2}$, as $\dot{V}_{R} \mathrm{O}_{2}$ is increased: these isopleths converge on the origin of the axes (Cerretelli and di Prampero 1987). Conversely, Fig. 11 shows $\mathrm{C}_{a} \mathrm{O}_{2}$ isopleths converging on a negative $x$-axis intercept, corresponding to $\dot{Q}_{\bar{v}} O_{2}$. During moderate exercise in normoxia, the $\dot{Q}$ versus $\dot{V}_{R} O_{2}$ relationship coincides with one $\mathrm{C}_{a} \mathrm{O}_{2}$ isopleth, that for the incurring $\mathrm{C}_{a} \mathrm{O}_{2}$, because $\mathrm{C}_{a} \mathrm{O}_{2}$ is essentially invariant.

The data shown in Fig. 10 are exercise data, a condition where the activity of the parasympathetic branch of the autonomic nervous system is blunted, and thus remarkably lower than that of the sympathetic branch (Ekblom et al. 1972; Fagraeus and Linnarsson 1976; Robinson et al. 1966). At rest, in which there is predominant vagal control of heart rate (Malliani et al. 1991; Perini and Veicsteinas 2003), the data lie below those obtained at exercise, whence a lower $\dot{Q}_{\bar{v}} O_{2}$ value. This agrees with the previous observations, both during exercise on the cycle ergometer (Anchisi et al. 2001; Ferretti et al. 2005) and during twolegged knee extension exercise (Koskolou et al. 1997a; Roach et al. 1999). A lower $\dot{Q}_{\bar{v}} O_{2}$ implies a right shift of the $\mathrm{C}_{a} \mathrm{O}_{2}$ isopleths in Fig. 11 at rest (see Adami et al. 2014, Fig. 2 of that article).
As long as we operate on the flat part of the oxygen equilibrium curve, these relationships still endure. One example is anaemic individuals (Koskolou et al. 1997b; Roach et al. 1999; Woodson et al. 1978) who are characterised by a reduced $\mathrm{C}_{a} \mathrm{O}_{2}$, because they have a low blood haemoglobin concentration in absence of $\mathrm{S}_{a} \mathrm{O}_{2}$ changes. These subjects would have a $\dot{Q}$ versus $\dot{V}_{R} O_{2}$ relationship coinciding with a steeper $\mathrm{C}_{a} \mathrm{O}_{2}$ isopleth on the $\dot{Q}-\dot{V}_{R} O_{2}$ diagram. The same would occur with carbon monoxide poisoning (GonzalezAlonso et al. 2001). Another, opposite, example is polycythaemia, whatever its cause (Celsing et al. 1986; Ekblom et al. 1976; Ferretti et al. 1990, 1992), during which the $\dot{Q}$ versus $\dot{V}_{R} O_{2}$ relationship would coincide with a flatter $\mathrm{C}_{a} \mathrm{O}_{2}$ isopleth. We expect this to be the case also following erythropoietin administration, but the experiment is yet to be done. In the only study we are aware of, on the effects of erythropoietin administration on oxygen transport during moderate exercise (Thomsen et al. 2007), the authors did not measure $\dot{Q}$. All these changes are compatible with the inverse relationship between $\dot{Q}$ and $\mathrm{C}_{a} \mathrm{O}_{2}$ (Calbet et al. 2006).

Conversely, when we operate on the steep part of the oxygen equilibrium curve, as in acute hypoxia, diffusion limitation appears in alveolar-capillary oxygen transfer, depending on exercise intensity. In fact, the shortening of $t_{c}$ induced by the increase in $\dot{Q}$ results in progressively lower $\mathrm{S}_{a} \mathrm{O}_{2}$ values at the arterial end of lung capillaries. Therefore, $\mathrm{C}_{a} \mathrm{O}_{2}$ in hypoxia becomes lower, the higher the $\dot{V}_{R} \mathrm{O}_{2}$ and $\dot{Q}$. The progressive $\mathrm{C}_{a} \mathrm{O}_{2}$ reduction shifts the $\dot{Q}$ versus $\dot{V}_{R} O_{2}$ relationship toward an isopleth for lower $\mathrm{C}_{a} \mathrm{O}_{2}$ values. This implies that (i) the apparent $\dot{Q}$ versus $\dot{V}_{R} O_{2}$ line has a higher slope than that for normoxia; and (ii) the same line points toward a higher $x$-axis intercept, indicative of an apparently lower $\dot{Q}_{\bar{v}} O_{2}$ value (Adami et al. 2014). Anchisi et al. (2001) found lower $\dot{Q}_{\bar{v}} \mathrm{O}_{2}$ the lower was the $\mathrm{S}_{a} \mathrm{O}_{2}$. They also found a positive linear relationship between these two parameters at exercise. This means that there is more than a mere displacement across $\mathrm{C}_{a} \mathrm{O}_{2}$ isopleths of the $\dot{Q}$ versus $\dot{V}_{R} \mathrm{O}_{2}$ relationship in hypoxia. There is also a right displacement of the $x$-axis intercept, implying a decrease of $\dot{Q}_{\bar{v}} O_{2}$.

Various mechanisms may explain the characteristics of the $\dot{Q}-\dot{V}_{R} O_{2}$ diagram. Oxygen sensing mechanisms may be important in this context. Thus, it would be easy to think, above all, of the peripheral chemoreceptor: it is an oxygen sensor, inhibited by oxygen binding, with a clear transduction mechanism and afferent pathway, it has connections to the integrated cardio-respiratory control centres in the brainstem, and a dense autonomic innervation (Brognara et al. 2021; Lahiri et al. 2001; Patel and Honoré 2001; Spyer and Gourine 2009; Wilson and Teppema 2016). The carotid body would be an almost ideal oxygen sensor for a systemic response like that proposed by Ferretti et al. 
(1992). However, the observation that also local skeletal muscle blood flow responds to changes in $\mathrm{C}_{a} \mathrm{O}_{2}$ calls for mechanisms that may operate also in the periphery, not only on central circulation.

The haemoglobin molecule was then proposed as an oxygen sensor (Calbet 2000; Singel and Stamler 2005), with a mechanism potentially mediated by its S-nitrosylation, with subsequent rise of nitric oxide in blood, leading to peripheral vasodilation (Stamler et al. 1997). As stated by Premont et al. (2020), hypoxic vasodilation is recapitulated by native S-nitrosothiol (SNO)-replete red blood cells and by SNO-haemoglobin itself, whereby SNO is released from haemoglobin and red blood cell during deoxygenation, in proportion to the degree of haemoglobin deoxygenation, to regulate vessels directly. Crecelius et al. $(2011 \mathrm{a}, \mathrm{b})$ proposed a synergistic effect of prostaglandins and nitric oxide in the regulation of peripheral blood flow during exercise in hypoxia. Alternatively, others have suggested that peripheral blood flow may be increased by ATP-mediated vasodilation in subjects with low $\mathrm{C}_{a} \mathrm{O}_{2}$ (Ellsworth et al. 1995; Gonzalez-Alonso et al. 2002; Mortensen et al. 2011). The mechanism proposed by Premont et al. (2020) is tempting, in so far as it transfers the sensing process to the level of local regulation of peripheral blood flow. However, if we admit such a mechanism, the inverse relationship between $\dot{Q}$ and $\mathrm{C}_{a} \mathrm{O}_{2}$ would be the non-deterministic result of a series of local actions on blood flow. It would make sense, rather, that a combination of central (carotid body stimulation) and peripheral (nitrosylation) mechanisms synergistically act to set the inverse relationship between $\dot{Q}$ and $C_{a} O_{2}$, and the constancy of $\dot{Q}_{\bar{v}} O_{2}$.

\section{On short-term pressure regulation}

The physical laws governing the flow of a liquid in a pipe system, wherein flow is sustained by a pressure gradient that is necessary to overcome resistance to flow, apply to the cardiovascular system, as well. Therefore, at a first approximation, and neglecting the right atrial pressure, which is close to zero, $\dot{Q}$ is also equal to the ratio of the mean arterial pressure $(\bar{P})$ to $R_{p}$. Thus, Eq. (5b) can be rewritten as follows:

$\dot{V}_{R} O_{2}=\frac{\bar{P}}{R_{p}}\left(C_{a} O_{2}-C_{\bar{v}} O_{2}\right)$.

This means that, ceteris paribus, $\dot{V}_{R} O_{2}$ is directly proportional to $\bar{P}$ and inversely proportional to $R_{p}$. Therefore, keeping a tight coupling of respiration and metabolism is also a matter of arterial blood pressure. Without considering medium-term and long-term mechanisms of pressure regulation, in view of their longer time scale than that of the phenomena we are dealing with, short-term regulation of blood pressure may have an important role in maintaining adequate oxygen flow and blood flow to match metabolic demand.

The baroreflex system is the main short-term control system of arterial blood pressure. It includes low-pressure (atrial receptors) and high-pressure (arterial receptors) afferent inputs. The former have to do mainly with volume regulation. The latter consist essentially of the arterial baroreflexes. They can be conceptually represented as follows: the stretching of mechanical receptors, recently identified by some authors as piezo ion channels ${ }^{8}$ (Coste et al. 2012; Murthy et al. 2017; Retailleau et al. 2015; Wang et al. 2016), located in the arterial wall at the common carotid bifurcation and in the aortic arch, generates action potentials that are conveyed by the glossopharyngeal and the vagus nerve, respectively, to the cardiac and vasomotor centres, where they determine an integrated inhibitory response. Two components of this response have been identified: a cardiac component, acting through a reduction of $f_{H}$ mediated by vagal stimulation of the heart; and a vascular component, acting through inhibition of vascular sympathetic efferents. Both components lead to a reduction of $\bar{P}$ (Eckberg and Sleight 1992).

The concept of arterial baroreflexes was first proposed by Marey (1863), who described a possible reflex regulating arterial blood pressure on a short-time basis. However, the observation that both $f_{H}$ and arterial blood pressure increase at exercise, appeared in contrast with the reflex nature of pressure control (Krogh and Lindhard 1913b). Their interpretation was that the reflex was switched off at exercise by a neural mechanism. Two hypotheses were put forward to this aim: the first was the so-called exercise pressor reflex (Zuntz and Geppert 1886) and the second was the so-called central command hypothesis (Johansson 1895). Krogh and Lindhard (1913b) retained the latter, inasmuch as the activation of the cardiovascular system occurred simultaneously with the exercise onset.

The most significant development in human baroreflex representation, with respect to Krogh's times, has been the introduction of the new concept of baroreflex resetting at exercise, which has replaced the concept of reflex switch-off. This change has introduced a new paradigm in our vision of baroreflexes at exercise. It was prompted by Bevegård and Shepherd (1966), who had been the first to use the neck suction-neck pressure technique on exercising humans. They showed that arterial baroreflexes were not switched off, but persisted during exercise, although

\footnotetext{
${ }^{8}$ It is fair to acknowledge that the piezo ion channel hypothesis on baroreceptors has been scrutinized and challenged by other authors (Stocker et al. 2019).
} 


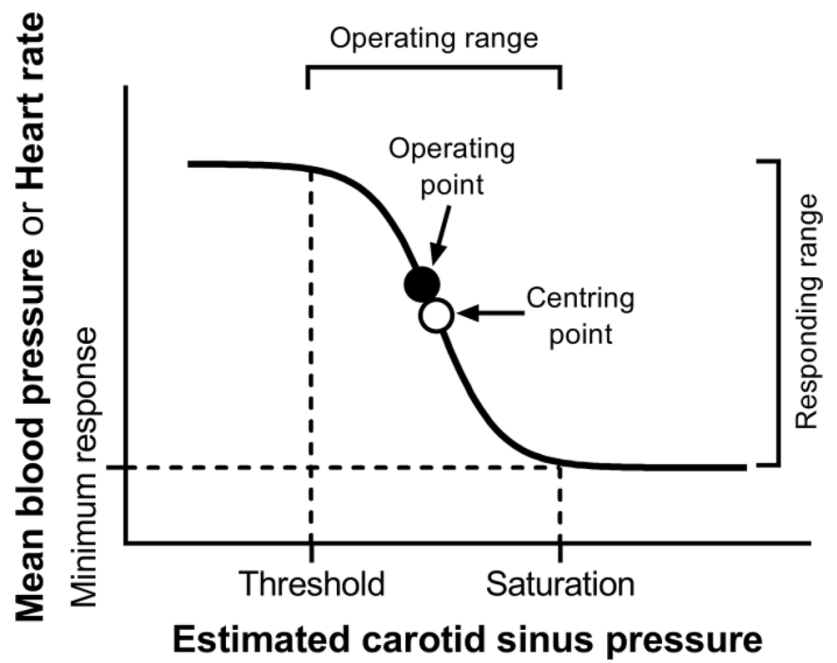

Fig. 12 Schematic representation of the carotid baroreflex function curve and its operational parameters, based on the logistic model of Kent et al. (1972). The responding range corresponds to the overall change in the dependent variable. The operating range is the pressure difference between the carotid sinus pressures at the threshold (carotid sinus pressure below which no further changes in heart rate or in arterial pressure take place) and saturation (carotid sinus pressure above which no further drop in heart or in arterial pressure occurs) of the reflex. The operating point (prevailing pressure and heart rate at steady state before the stimulus) and the centring point (the point midway between the threshold and saturation pressures) are also shown. At rest, they are very close to each other. The maximal gain is the gain value at the centring point [Modified after Raven et al. (2006)]

in a different pressure range from rest. Moreover, that study set the basis for further investigations (Bristow et al. 1969, 1971; Cunningham et al. 1972; Pickering et al. 1971, 1972), which led Kent et al. (1972) to construct the logistic model of arterial baroreflexes. This model and its parameters are described in Fig. 12. The operating point

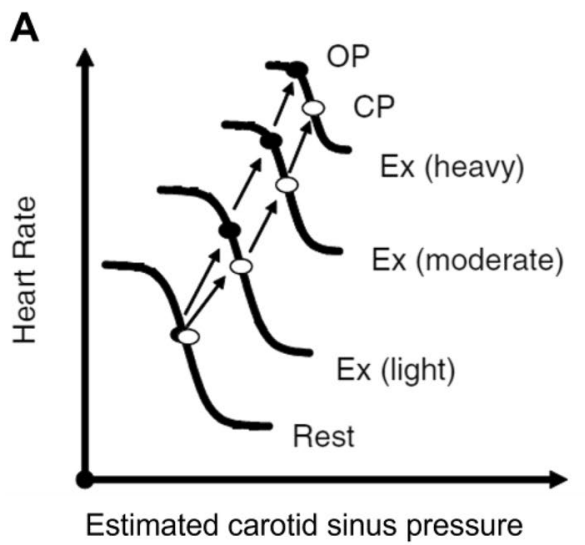

Fig. 13 Representation of the carotid-cardiac (panel A) and carotidvasomotor (panel B) baroreflex resetting, that occurs from rest to heavy exercise. It appears that (i) the entire baroreflex response curve at exercise is progressively displaced upward and rightward without corresponds to the prevailing $\bar{P}$ and $f_{H}$, around which the system operates at steady state. The maximal baroreflex gain is defined as the gain value at the centring point of a baroreflex curve (see Raven et al. 2006, for details).

The baroreflex curves at steady-state exercise were constructed in several studies, using the neck suction-neck pressure technique (Di Carlo and Bishop 1992; Norton et al. 1999a, b; Papelier et al. 1994; Potts et al. 1993). All those studies demonstrate that a displacement upward and rightward of the entire baroreflex curve occurs during exercise indeed: this displacement was defined as baroreflex resetting at exercise. Normally, baroreflex resetting occurs without changes in maximal gain, but with a shift of the operating point far from the centring point, toward the threshold pressure (Fadel and Raven 2012; Raven et al. 2006). A representation of baroreflex resetting during exercise is reported in Fig. 13. Baroreflex resetting was observed also in conditions, such as heat exposure (Crandall 2000), postural changes (Linnarsson et al. 2006; Ogoh et al. 2007), and simulated microgravity (Convertino et al. 1994; Linnarsson et al. 2006).

Despite the huge amount of work carried out since Krogh and Lindhard (1913b), the two hypotheses of a central command and of an exercise pressor reflex are still at the core of the debate on arterial baroreflexes as possible mechanisms behind baroreflex resetting at exercise (Fadel and Raven 2012; Fisher et al. 2015; Gallagher et al. 2006; McIlveen et al. 2001; Michelini et al. 2015; Raven et al. 2006, 2019). In fact these hypotheses are not mutually exclusive, and both were included in a comprehensive theory of baroreflex resetting at exercise (Degtyarenko and Kaufman 2006; Tsuchimochi et al. 2009).

The neck suction-neck pressure technique provides a static analysis of arterial baroreflexes, which requires a steady state in the cardiovascular system, upon which an

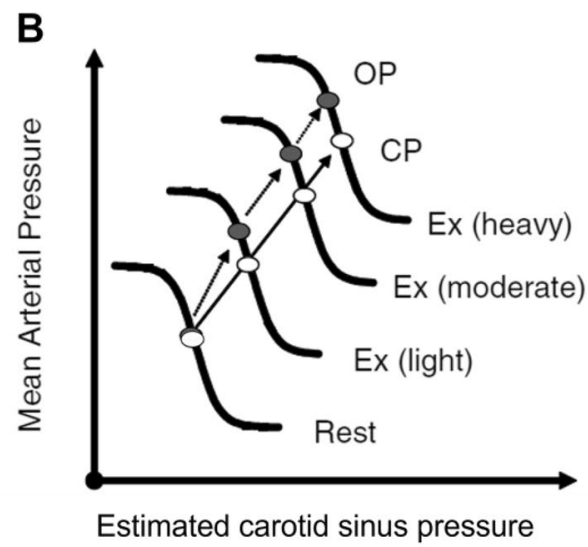

changes in slope, and (ii) the operating point progressively shifts far from the centring point, toward the threshold pressure. Ex exercise in the indicated intensity domain, $C P$ centring point, $O P$ operating point [From Raven et al. (2006)] 
external perturbation (open-loop approach) of varying intensity is introduced to modify the carotid distending pressure (independent variable); the ensuing $f_{H}$ or $\bar{P}$ responses are then recorded as dependent variables. This approach has led to thorough advancement in our baroreflex understanding, yet it has a limitation as to the understanding of the mechanisms behind resetting. Since it requires steady state, no dynamics of resetting can be studied with open-loop methods, despite awareness that resetting occurs during a dynamic condition, namely the transition between rest and exercise, or between two different exercise intensities. Nevertheless, baroreflex resetting in response to an external perturbation (exercise, for example) implies a transient phase, which occurs between the previous and the new steady state, and this was not accounted for in the open-loop approach. This is one of the most important reasons why the respective roles of central command and the exercise pressor reflex remain to be precisely defined. At present, the only chance of a deeper understanding of the mechanisms determining baroreflex resetting between two different steady states may come from an analysis of the temporal relationships during the dynamic phase of resetting. This analysis requires comprehensive studies of the kinetics of displacement of the baroreflex curve, or at least of its operating point, and therefore cannot be carried out with an open-loop approach.

Arterial baroreflexes can be investigated also by a different, less comprehensive way than the open-loop approach: the closed-loop analysis, wherein continuous beat-by-beat follow-up of the $f_{H}$ (or its reciprocal, the RR-interval, $R R i$ ) and blood pressure changes, assuming a continuous effect of blood pressure on $f_{H}$ with immediate counter-effect of $f_{H}$ on pressure. The most classical closed-loop method in the time domain is the sequence method (Bertinieri et al. 1988). This method is applied on sequences of at least three consecutive beats characterised by consensual variations of $R R i$ and of arterial pressure. Within each sequence, Bertinieri et al. (1988) treated the $R R i$ versus blood pressure relationship as linear, and assumed that the slope of this relationship represents the spontaneous baroreflex sensitivity around the operating point. It is noteworthy that, in conditions in which central blood volume, and thus mean arterial pressure, are increased, such as the supine posture (O'Leary et al. 2003; Steinback et al. 2005), water immersion (Chouchou et al. 2020), and short-term microgravity exposure (Di Rienzo et al. 2008; Hirayanagi et al. 2004), baroreflex sensitivity is also increased.

Conversely, application of the sequence method to the study of baroreflex sensitivity at rest and during exercise steady state has shown that the baroreflex sequences at exercise do not lie on an $R R i$ versus pressure plot in the same position as at rest, but appear displaced downward and rightward, in agreement with the baroreflex resetting hypothesis
(Bringard et al. 2017; Iellamo et al. 1994, 1997, 2002; Vallais et al. 2009). Moreover, the baroreflex sensitivity at exercise is remarkably reduced. The steady-state baroreflex curves reported in Fig. 13 show that the baroreflex operating point is displaced, along the logistic baroreflex curve, away from the centring point, toward the threshold pressure. This explains the finding of lower baroreflex sensitivity at exercise, as long as the slope of the logistic curve of Kent et al. (1972) becomes flatter as the threshold is approached.

Bringard et al. (2017) investigated the dynamics of baroreflex resetting with a closed-loop approach. Their data show that no baroreflex resetting was evident at exercise onset, as long as a progressive consensual increase in $f_{H}$ and in $\bar{P}$ up to the steady-state values appeared only after a minimum level of $\bar{P}$ had been attained, which occurred after some $10 \mathrm{~s}$ of exercise. In the first seconds of exercise, they found a linear negative segment of the $f_{H}$ and in $\bar{P}$ relationship, which they treated a sequence of heart beats, as defined by Bertinieri et al. (1988). The slope of this segment was equal to the baroreflex sensitivity during exercise, but significantly lower than the baroreflex sensitivity at rest. The initial linear segment appears as a baroreflex response to the sudden fall of $\bar{P}$ induced by muscle vasodilation at exercise onset, which occurs simultaneously with the first muscle contraction (Saltin et al. 1998). Conversely, the delayed onset of baroreflex resetting seems incompatible with the two hypotheses of central command and exercise pressor reflexes as its possible determinants. Notwithstanding, we cannot dismiss central command as a potential mechanism behind the reduction of baroreflex gain at exercise onset, in view of its immediacy. Similar conclusions were more recently attained by applying the same approach to the analysis of baroreflex changes during breath-holding (Taboni et al. 2021a) and during tilting (Taboni et al. 2021b).

\section{Matching things at steady state}

After having analysed the main relationships that at steady state represent the cardio-respiratory responses to exercise, which are tightly coupled with the metabolic responses to exercise, in this section, we propose a synthesis of the various relationships that we have discussed in previous paragraphs. To do so, let us return to constant $\dot{Q}_{\bar{v}} O_{2}$, corresponding the $x$-axis intercept of a $\dot{Q}$ versus $\dot{V}_{R} O_{2}$ line, as outlined by Eq. (31) and in Fig. 11. At the same time, the solution for $\dot{Q}$ of the ventilation-perfusion equation (Eq. 22) is 
$\dot{Q}=-\frac{\dot{V}_{A} P_{A} C O_{2}}{C g R Q_{L}\left(C_{a} O_{2}-C_{\bar{v}} O_{2}\right)}$.

Since Eqs. (31) and (33) must have equal solutions at steady state, we can also write

$\frac{\dot{V}_{R} O_{2}+\dot{Q}_{\bar{v}} O_{2}}{C_{a} O_{2}}=-\frac{\dot{V}_{A} P_{A} C O_{2}}{C g R Q_{L}\left(C_{a} O_{2}-C_{\bar{v}} O_{2}\right)}$.

Since

$\mathrm{C}_{a} \mathrm{O}_{2}=[\mathrm{Hb}] \sigma \mathrm{S}_{a} \mathrm{O}_{2}$,

where $\sigma$ is the oxygen binding capacity of haemoglobin and $[H b]$ is blood haemoglobin concentration ${ }^{9}$. Equation (34) can thus be rewritten as follows:

$\dot{V}_{R} O_{2}+\dot{Q}_{\bar{v}} O_{2}=-\frac{[H b] \sigma S_{a} O_{2} \dot{V}_{A} P_{A} C O_{2}}{C g R Q_{L}\left(C_{a} O_{2}-C_{\bar{v}} O_{2}\right)}$.

For humans at sea level, since $C g=0.863 \mathrm{mmHg}$ and $\sigma=1.34 \mathrm{~mL}$ of oxygen per gram of haemoglobin, solving Eq. (36) for $\dot{V}_{R} O_{2}$ would provide

$\dot{V} O_{2}=\dot{V}_{R} O_{2}=-\frac{1.552[\mathrm{Hb}] \mathrm{S}_{a} \mathrm{O}_{2} \dot{V}_{A} P_{A} \mathrm{CO}_{2}}{R Q_{L}\left(C_{a} O_{2}-C_{\bar{v}} O_{2}\right)}-\dot{Q}_{\bar{v}} O_{2}$.

The most complete set of data reporting resting respiratory and cardiovascular values at sea level is the Operation Everest II, a huge study carried out in the 1980s wherein an ascension to Mount Everest was simulated in a hypobaric chamber and, among others, a systematic survey of the overall oxygen transport system was performed (Houston et al. 1987). At rest in normoxia, the subjects of Operation Everest II (Sutton et al. 1988) had $P_{A} \mathrm{CO}_{2}=33.9 \mathrm{mmHg}$ (arterial pH 7.43) and $\dot{V} O_{2}=\dot{V}_{R} O_{2}=0.35 \mathrm{~L} \mathrm{~min}^{-1}$. For the other variables pertaining to Eq. (37), ${ }^{10}$ we estimated: $[H b]=135 \mathrm{~g} \mathrm{~L}^{-1}, S_{a} O_{2}=0.976, \dot{V}_{A}=5.97 \mathrm{~L} \mathrm{~min}^{-1}$ (calculated from $\dot{V}_{R} C O_{2}$, and $\left.P_{A} C O_{2}\right), R Q_{L}=0.771, C_{a} O_{2}=179 \mathrm{~mL} \mathrm{~L}^{-1}$, and $C_{\bar{v}} O_{2}=122 \mathrm{~mL} \mathrm{~L}^{-1}$. This would have implied for them a $\dot{Q}_{\bar{v}} O_{2}$ of $0.50 \mathrm{~L} \mathrm{~min}{ }^{-1}$, to be compared with a measured $\dot{Q}_{\bar{v}} O_{2}$ of $0.76 \mathrm{~L} \mathrm{~min}^{-1}$. Considering that the subjects of Operation Everest II had a low resting $[H b]$ at sea level, which lowers the $\dot{Q}_{\bar{v}} O_{2}$ estimated after Eq. (37), and a high resting $\dot{Q}$ $\left(6.3 \mathrm{~L} \mathrm{~min}^{-1}\right)$, which keeps their measured $\dot{Q}_{\bar{v}} \mathrm{O}_{2}$ up, this is a remarkable correspondence; indeed, the discrepancy being

\footnotetext{
${ }_{9}$ Equation (35) does not include dissolved oxygen, which at a $\mathrm{P}_{a} \mathrm{O}_{2}$ of $100 \mathrm{mmHg}$ contributes only $3 \mathrm{~mL}$ of oxygen per litre of blood, i.e., about $1.5 \%$ of the overall amount of oxygen in arterial blood: a negligible quantity indeed.

${ }^{10}$ In this case, expressing $[\mathrm{Hb}]$ in $\mathrm{g} \mathrm{L}^{-1}, \dot{V}_{A}$ in $\mathrm{L} \mathrm{min}^{-1}, P_{A} \mathrm{CO}_{2}$ in $\mathrm{mmHg}$ and $\mathrm{C}_{a} \mathrm{O}_{2}-\mathrm{C}_{\bar{v}} \mathrm{O}_{2}$ in $\mathrm{mL} \mathrm{L}^{-1}, \dot{V}_{R} O_{2}$ turns out in $\mathrm{mL} \mathrm{min}^{-1}$.
}

essentially explained by interindividual data variability and by computation assumptions.

More generally speaking, Eq. (36) defines how, in a steady-state condition, the characteristic variables describing the functional status of the oxygen transport system may adjust to variations of metabolic oxygen requirements, sustaining body homeostasis. Except for $\sigma$ and for $C g$, which are predefined constants independent of metabolism, many combinations of these variables are possible; only a few, in contrast, actually occur, depending on the settings of the various control mechanisms that operate in maintaining homeostasis. These settings are such as to result in a fairly stable $\dot{Q}_{\bar{v}} O_{2}$.

In normoxia, in which $P_{a} \mathrm{CO}_{2}(40 \mathrm{mmHg})$ and $\mathrm{C}_{a} \mathrm{O}_{2}$ (200 $\mathrm{mL} \mathrm{L}^{-1}$ for normal $[\mathrm{Hb}]$ ) may be considered essentially invariant during rest and steady-state submaximal exercise, any increase in $\dot{V} O_{2}$, requires an increase in both $\dot{V}_{A}$ and $\dot{Q}$. In fact, $\dot{V}_{A}$ is proportional to $\dot{V}_{R} O_{2}$ only if $R Q_{L}$ does not change, whereas $\dot{Q}$ is not proportional to $\dot{V} O_{2}$. In hypoxia, when diffusion limitation appears, both in lungs and in muscles (Piiper and Scheid 1981; Piiper 2000), $C_{a} O_{2}$ drops, $\dot{Q}$ only partially corrects this drop, the constant $\dot{Q}_{\bar{v}} O_{2}$ takes a lower value, and $\dot{V}_{A}$ goes up due to stimulation of peripheral chemoreceptors. All relations to $\dot{V}_{R} O_{2}$ are modified, and thus, the entire homeostasis of the respiratory system at exercise is modified: the new equilibrium is attained at $P_{a} \mathrm{CO}_{2}$ values lower than $40 \mathrm{mmHg}$. We would expect a new equilibrium around lower $P_{a} \mathrm{CO}_{2}$ values also when $b \dot{\mathrm{La}}$ becomes positive and hyperventilation superimposes due to stimulation also of central chemoreceptors. This new steady state, however, is never attained, as we discuss below.

The next paragraphs will be devoted to analyse some major cases of rupture of steady-state homeostatic equilibria, implying potential disruption of the tight coupling of respiration and metabolism.

\section{Breaking the equilibrium: the exercise transient}

When a human starts a constant-power exercise of moderate intensity, the resting equilibrium is broken and a transition between the resting and the exercise steady states takes place. Although the application of mechanical power is immediate, $\dot{V} O_{2}$, which at the steady state of aerobic exercise is equal to $\dot{E}$, increases at a remarkably slower rate. The steady-state $\dot{V} \mathrm{O}_{2}$ is attained in approximately $3 \mathrm{~min}$. During this transient phase, $\dot{V} \mathrm{O}_{2}$ is insufficient to sustain the overall ATP resynthesis at the necessary rate to cope with the rate of ATP hydrolysis during muscular contraction. Therefore, metabolic power must be generated also by anaerobic metabolisms. 
An energetic view of the $\dot{V} O_{2}$ kinetics upon exercise onset is discussed elsewhere (di Prampero 1981; Ferretti 2015). Here, we merely underline that, coherently with the principles set by Margaria et al. (1933), the $\dot{V} O_{2}$ kinetics was described as a mono-exponential

$\dot{V} O_{2}{ }^{s}-\dot{V} O_{2}{ }^{t}=\dot{V} O_{2}{ }^{s} e^{-t \tau^{-1}}$,

where $\dot{V} O_{2}{ }^{t}$ is the $\dot{V} O_{2}$ at time $t$ during the transient, $\dot{V} O_{2}{ }^{s}$ is the $\dot{V} O_{2}$ at steady state, and $\tau$ is the time constant describing the exponential equation. Within such a model, the overall oxygen deficit $\left(\mathrm{DO}_{2}\right)$ can be computed as the time integral of Eq. (38)

$D O_{2}=\int_{0}^{\infty} \dot{V} O_{2}^{s} e^{-t \tau^{-1}} d t=\tau \dot{V} O_{2}^{s}$.

In Margaria's energetic view, the oxygen deficit is the amount of energy derived from energy sources different from aerobic metabolism during the exercise transient.

The $\dot{V} O_{2}$ kinetics was generally determined from $\dot{V}_{R} O_{2}$ measurements at the mouth. This procedure implies the assumption that the kinetics of $\dot{V}_{R} O_{2}$ corresponds to the kinetics of $\dot{V} \mathrm{O}_{2}$ taking place in the working muscles, once possible changes in blood oxygen stores have been accounted for. This assumption carries along the corollary that the $\dot{V} O_{2}$ and $\dot{V}_{R} O_{2}$ kinetics have equal time constants, so that they remain tightly coupled also during the exercise transient. A second implicit fundamental assumption is that the muscle metabolic response to exercise onset follows the laws of linear first-order systems. Although this may indeed be the case during moderate exercise, it is definitely not so during higher intensity exercise. The energy balance of the $\dot{V} \mathrm{O}_{2}$ kinetics based on these principles was originally analysed by di Prampero and Margaria (1968).

Several authors, however, considered this view too simplistic. The gap between the contracting muscles and the mouth is so wide, that it was hard to believe in a system as simple and linear as that proposed by di Prampero and Margaria (1968). Gilbert et al. (1967) were the first to suggest a rapid component of the $\dot{V}_{R} O_{2}$ kinetics at exercise onset. This finding was soon confirmed and analysed in detail (Whipp and Wasserman 1972). Wasserman et al. (1974), with experiments on anesthetized resting dogs, in which lung blood flow was artificially modified, demonstrated the possibility that the rapid component of $\dot{V}_{R} O_{2}$ changes in the exercise transient be due to a sudden increase in $\dot{Q}$. These findings suggested that a mono-exponential model might be insufficient to describe the $\dot{V}_{R} O_{2}$ kinetics at the onset of a squarewave exercise. The increasing evidence led to the creation of the double-exponential model with time delay of the $\dot{V}_{R} O_{2}$ kinetics (Barstow and Molé 1987). The arrival of this model questioned the tight matching of $\dot{V} O_{2}$ to $\dot{V}_{R} O_{2}$ during the exercise transient. In fact, the domain of application of the metabolic vision of the oxygen deficit was restricted to the second exponential only, which some authors later defined as the primary component of the $\dot{V}_{R} O_{2}$ kinetics.

It is interesting to note that, when Whipp and Wasserman (1972) analysed the rapid phase of the $\dot{V}_{R} O_{2}$ kinetics at exercise onset, several authors had already recognized a fast component in the dynamics of pulmonary ventilation (Asmussen 1973; Asmussen and Nielsen 1948; Astrand and Christensen 1963; Comroe and Schmidt 1943; D'Angelo and Torelli 1971; Dejours 1964; Krogh and Lindhard 1913b). This generated the notion of dissociation of ventilatory control from metabolic control, inasmuch as the increase in ventilation was to precede the increase in $\dot{V} O_{2}$ (or in $\dot{V}_{R} O_{2}$ ). On this basis, many authors agreed that neurogenic mechanisms were behind the rapid ventilatory response at exercise onset, independent of whether a central command or a peripheral reflex mechanism was involved (Comroe 1944; Dejours 1964; Eldridge et al. 1981; Favier et al. 1983; McCloskey and Mitchell 1972; Turner 1991; Whipp 1994; Whipp and Ward 1982).

The question as to whether the time course of $\dot{V}_{R} O_{2}$ upon exercise onset is conveniently described by a two-phase, rather than a single-phase, $\dot{V}_{R} O_{2}$ kinetics, implying that the first rapid phase is dissociated from metabolic control, has been one of the fundamental questions in exercise physiology in the last 40 years (for a summary of the issue, see Poole and Jones 2012; Ferretti 2015). This question carries along another, directly related question, of much more pertinence in the present context. We can formulate it as follows: taking for granted that a rupture of a steady equilibrium occurs whenever we move from a steady state to a different steady state, implying a readjustment of most of the key parameters of Eq. (36), is this readjustment fast enough to cope with the increasing oxygen demand? If it is, a tight coupling of respiration to metabolism is maintained even during the exercise transient; if it is not, this tight coupling is transiently broken, to be re-established when the new steady state has been attained. These two questions are relatively new. Originally, the oxygen deficit was analysed only from an energetic viewpoint, and it may be useful to start from an analysis of the energetics of the oxygen deficit also to answer the above questions.

\section{The energetics of the oxygen deficit}

The interest of Margaria and his pupils was in understanding the question "where does the energy during the exercise transient come from". Their answer was: from alactic and lactic anaerobic metabolisms. The former was considered an obligatory component of the oxygen deficit and was the starting point of the analysis of di Prampero and Margaria 


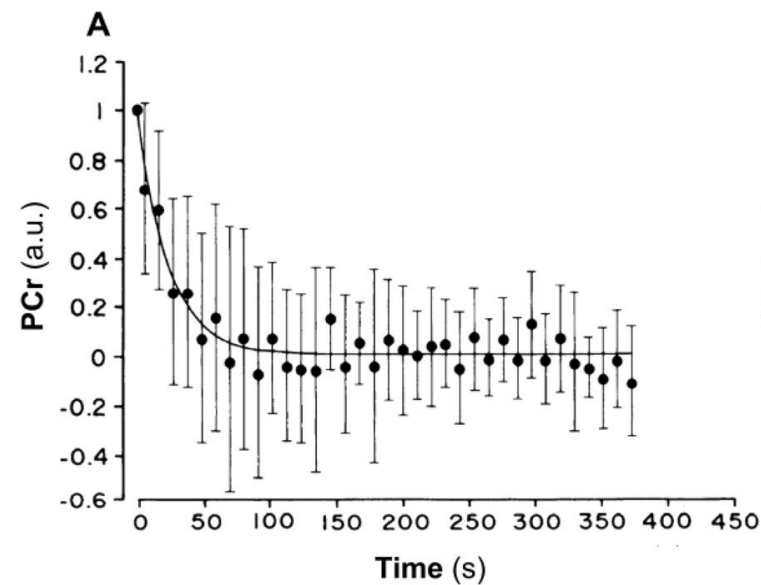

Fig. 14 A time course of phosphocreatine concentration changes upon exercise onset, as determined by ${ }^{31} \mathrm{P}$ nuclear magnetic resonance. The exponential function has a time constant of $23.4 \mathrm{~s}$. Bars

(1968); the latter appears in the intense exercise domain, to cover a gap between speed of activation of glycolysis, on one side, and of aerobic metabolism on the other (early lactate, Cerretelli et al. 1979).

They were also interested in the mechanism of the oxygen deficit. The main regulatory enzyme of glycolysis is phospho-fructo-kinase (PFK). As exercise starts, muscle ATP is immediately consumed in muscle contraction, so its concentration in contracting muscles tends to diminish. The first, rapid source of ATP resynthesis is the Lohmann reaction, the equilibrium constant of which is so elevated (between 20 and 100 , depending on hydrogen ion and magnesium concentrations, see Carlson and Siger 1959; Kuby et al. 1954), that it prevents in fact a measurable ATP fall. PCr decreases and free creatine and Pi go up. As a consequence, PFK activity is accelerated. The energy flux along the glycolytic pathway increases and prompts the aerobic metabolism overall.

If, based on the preceding information, we assume that the metabolic aerobic pathway, and thus muscle $\dot{V} O_{2}$, is in some way controlled by the muscle $\mathrm{PCr}$ concentration, or, rather, by its mirror image, free creatine, the fraction of the alactic oxygen deficit related to $\mathrm{PCr}$ hydrolysis $\left(D O_{2 A L}\right)$ controls muscle metabolism in the exercise transient (di Prampero and Margaria 1968; di Prampero et al. 2003; Mader 2003; Mahler 1985). This implies that the fraction of the oxygen deficit represented by $D O_{2 A L}$ is a physiological necessity to cope with the increase of ATP consumption at the onset of muscle contraction: in the absence of the alactic oxygen deficit, the muscle could not increase its metabolism. In this vision, the physiological significance of the Lohmann reaction is brought to a higher level: $\mathrm{PCr}$ is not only the molecule yielding energy for muscular contraction instantaneously, but is also the key controller of aerobic metabolism (di Prampero 1981; Francescato et al. 2008; Greenhaff

\section{B}

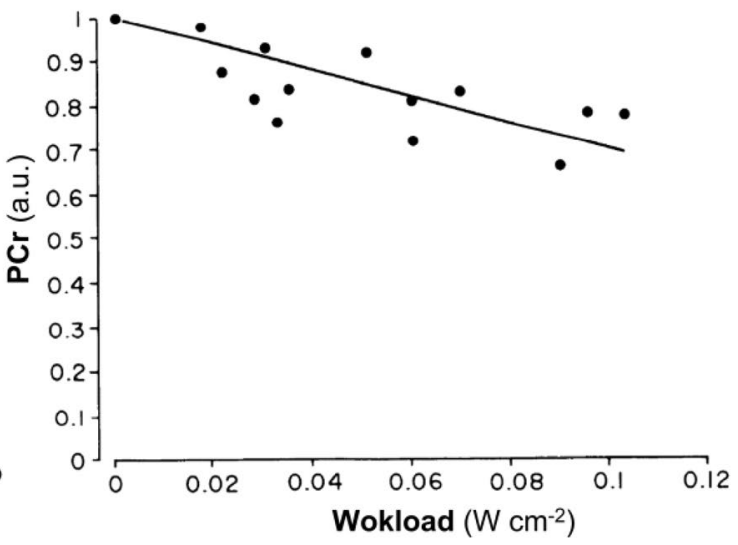

indicate standard deviation. B Linear relationship between steadystate phosphocreatine and power, from the same experiment [Modified after Binzoni et al. (1992)]

2001; Meyer et al. 1984; Meyer 1988; Rossiter et al. 2005; Walsh et al. 2001; Whipp and Mahler 1980). This view is also supported by the experiments of Grassi and coworkers on the isolated-perfused muscle preparation. Grassi et al. (2011) concluded: "The bioenergetic mechanism, which is fast enough to meet sudden increases in metabolic demand, that is PCr breakdown, is functionally related, through the level of some of its metabolites, to the regulation of oxidative phosphorylation, the most important mechanism for ATP resynthesis." For detail and further references on this and related matters, the reader is referred to Grassi et al. (2002, 2005).

A necessary consequence of the vision of the oxygen deficit outlined above is that the muscle $\dot{V} O_{2}$ kinetics at the onset of contraction is a first-order kinetics, as proposed by di Prampero and Margaria (1968). This implies a linear relationship with negative slope between $\dot{V} O_{2}$ and $\mathrm{PCr}$ at steady state (Cerretelli et al. 1969; di Prampero and Margaria 1968). Such a relationship was originally demonstrated on the isolated-perfused muscle preparation during stimulation of various intensities (Piiper et al. 1968), and confirmed in humans by means of ${ }^{31} \mathrm{P}-\mathrm{NMR}$ (nuclear magnetic resonance) experiments (Binzoni et al. 1992). As a corollary of this linear relationship, note that the maximal anaerobic alactic power and capacity, since they depend on muscle $\mathrm{PCr}$ concentration, are lower the higher is the steady-state $\dot{V} \mathrm{O}_{2}$ starting from which the test is performed (Margaria et al. 1971; Ferretti et al. 1987). Moreover, in the absence of early lactate accumulation in the contracting muscle mass, the kinetics of muscle $\mathrm{PCr}$ at exercise onset must have the same time constant as that of muscle $\dot{V} O_{2}$ (Binzoni and Cerretelli 1991). Several human studies using ${ }^{31} \mathrm{P}-\mathrm{NMR}$ spectroscopy, in which the latter time constant was calculated (Fig. 14), provided results coherent with both these predictions 
(Binzoni et al. 1992; di Prampero et al. 2003; Francescato et al. 2008, 2013; Rossiter et al. 1999, 2002; Whipp et al. 1999). Binzoni et al. (1992) reported a time constant $(\tau)$ for a mono-exponential $\mathrm{PCr}$ kinetics at exercise onset of $23.4 \mathrm{~s}$. This value was substantially confirmed by the subsequent cited studies. Moreover, Behnke et al. (2002) obtained a $\tau$ of intramuscular $\dot{V} \mathrm{O}_{2}$ kinetics of almost the exact same value, using phosphorescence quenching and intravital microscopy within contracting rat muscle.

The $\tau$ value of the PCr kinetics is also independent of the exercise intensity (Binzoni et al. 1997), so that the cumulative oxygen deficit of step-increasing powers up to a given level is equal to the oxygen deficit incurred if that very same level was attained in a single step. Moreover, the oxygen deficit is directly proportional to the steady-state $\dot{V} O_{2}$ with $\tau$ as proportionality constant, as shown by Eq. (39). Finally, further experiments on the isolated-perfused muscle preparation showed that, when muscle blood flow is artificially increased at rest or rapidly accelerated at exercise onset, no reduction in the time constant of the kinetics of $\dot{V} \mathrm{O}_{2}$ occurs (Grassi et al. 1998).

The muscle biochemical condition outlined here above typically corresponds to that of moderate aerobic exercise in normoxia, when no early lactate accumulation takes place and where body oxygen stores do not vary. The entire amount of energy released to build the oxygen deficit comes from $\mathrm{PCr}$ hydrolysis, as originally postulated by di Prampero and Margaria (1968). In this condition, the general equation of the energetics of muscular exercise (Eq. 2) takes the following simplified form

$\dot{E} \propto \overleftarrow{A \dot{T} P}=\overrightarrow{A \dot{T} P}=c \dot{V} O_{2}+a P \dot{C} r$

During the exercise transient of a constant-power exercise, as long as $\dot{V} O_{2}$ increases, $P \dot{C} r$ decreases in such a way as to maintain the sum $c \dot{V} O_{2}+a P \dot{C} r$ invariant. Within the context of a first-order kinetics system, and thus assuming a mono-exponential model of the $\dot{V} \mathrm{O}_{2}$ increase inside the contracting muscles, at exercise time 0 , all the $\dot{E}$ above the resting metabolic rate is from anaerobic alactic metabolism, so that Eq. (2) becomes

$\dot{E}_{0} \propto \overleftarrow{A \dot{T} P}=\overrightarrow{A \dot{T} P}=a P \dot{C} r_{0}$

where suffix 0 indicates the exercise time 0 . This being so, then we have

$a P \dot{C} r_{t}=a P \dot{C} r_{0} e^{-t \tau^{-1}}$,

where $t$ is time and $\tau$ is equal to that of the $P \dot{C} r$ kinetics. The time integral of Eq. (42) provides the overall amount of PCr hydrolysed during the exercise transient, constituting the oxygen deficit. This amount is directly proportional to the oxygen deficit, and thus to the applied mechanical power, whence the necessarily linear negative relationship between PCr concentration and $\dot{E}$.

A condition like that summarized in Eq. (40) is met if, and only if, the $\tau$ of the primary component of the $\dot{V}_{R} O_{2}$ kinetics is equal to, or faster than, the $\tau$ of $\dot{V} O_{2}$ in the contracting muscle mass. If this condition is met, and here we come to the main point in the present context, the rupture of the equilibrium leading to the new steady state at a higher $\dot{V} O_{2}$ does not imply uncoupling of $\dot{V}_{R} O_{2}$ and $\dot{V} O_{2}$. And this is so, no matter whether the primary component of $\dot{V}_{R} O_{2}$ and the muscle $\dot{V} O_{2}$ are modulated by the same control system, or are under different yet parallel control systems. This means that, whenever ATP consumption is increased by contraction, and thus, $\mathrm{PCr}$ falls and $\mathrm{Pi}$ and creatine go up, and the aerobic metabolic pathway has accelerated its rate, thus requiring a higher $\dot{V} O_{2}$, the respiratory system has simultaneously responded in such a way as to adapt $\dot{V}_{R} O_{2}$ to the increased oxygen demand.

In fact, more recent studies have shown that the $\tau$ of the primary component of the $\dot{V}_{R} O_{2}$ kinetics, determined with Grønlund's algorithm for breath-by-breath $\dot{V}_{R} O_{2}$ analysis (Capelli et al. 2001; Grønlund 1984), is faster (smaller) (Cautero et al. 2002, 2005; Lador et al. 2006, 2013) than that of the $\dot{V}_{R} O_{2}$ kinetics obtained in the classical studies (di Prampero 1981; Linnarsson 1974) carried out using the Auchincloss algorithm (Auchincloss et al. 1966), as a consequence of improved signal-to-noise ratio in breath-bybreath $\dot{V}_{R} O_{2}$ computation (Capelli et al. 2001). Therefore, it also turns out faster than that of muscle $\dot{V} O_{2}$ kinetics derived from muscle PCr kinetics (Binzoni et al. 1992; di Prampero et al. 2003; Rossiter et al. 1999), despite the fact that $\dot{V}_{R} O_{2}$ kinetics includes also the effects of changes in oxygen stores, which tend to increase $\tau$ and thus the corresponding oxygen deficit. Although the fast $\tau$ values obtained with Grønlund's algorithm suggest that the $\dot{V}_{R} O_{2}$ kinetics may not correspond to the $\dot{V} O_{2}$ kinetics, this implies, neither that the energetic interpretation of the oxygen deficit is incorrect, nor that $\dot{V}_{R} O_{2}$ is unable to match peripheral oxygen demand. This means only that any quantitative analysis of the oxygen deficit performed on the basis of a $\dot{V}_{R} O_{2}$ kinetics analysed with Grønlund's algorithm may provide an underestimate with respect to the oxygen deficit incurred in the contracting muscles, even accounting for the effects of oxygen store changes (Davies et al. 1972; di Prampero et al. 1970, 1983), which tend to increase the oxygen deficit. We maintain that, whenever the $\tau$ of the primary component of the $\dot{V}_{R} O_{2}$ kinetics is lower (faster) than, or at most equal to that of the $\dot{V} O_{2}$ kinetics, the tight coupling of $\dot{V}_{R} O_{2}$ and $\dot{V} O_{2}$ subsists, and Eq. (40) applies, even though the oxygen deficit computed from the former is actually smaller than that corresponding to the latter. Therefore, at least in the case of moderate aerobic exercise, breaking a steady-state 


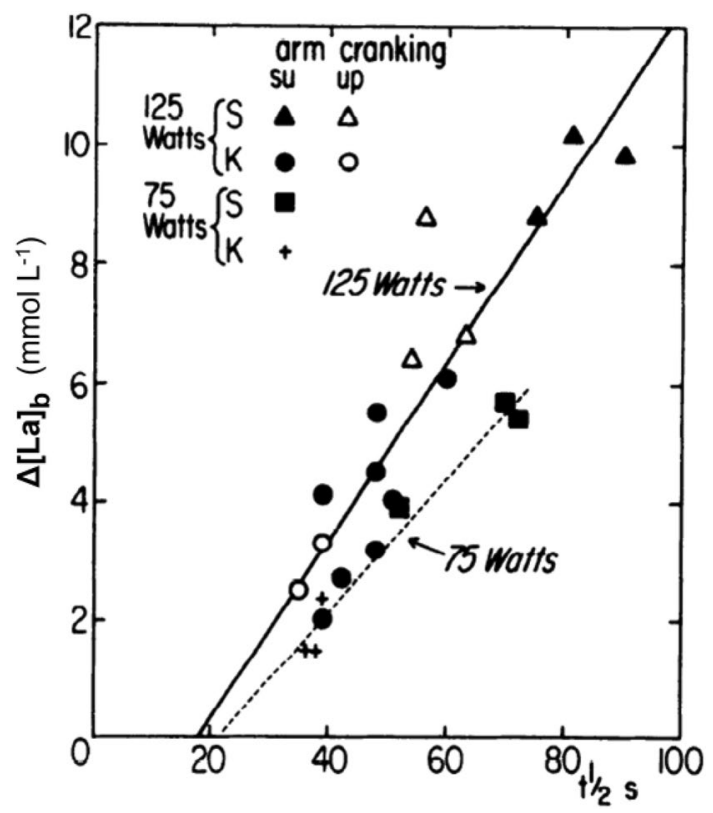

Fig. 15 Net blood lactate accumulation $\left(\Delta[L a]_{b}\right)$ at min 6 of squarewave exercises, as indicated on the graph, as a function of the halftime $\left(t^{1 / 2}\right)$ of the mono-exponential oxygen uptake increase upon exercise onset. Note that $t^{1 / 2}=0.69 \tau$. Note also that the representation proposed in this figure corresponds to the solution of Eq. (43) for $[L a]_{e}\left(\Delta[L a]_{b}\right.$ in the figure). In this representation, the constant $\tau_{0}$ of Eq. (43) corresponds to the $x$-axis intercept in the figure (yet expressed as $t^{1 / 2}$ instead of $\tau$ ). $S$ sedentary, $K$ kayakers [From Cerretelli et al. (1979)]

equilibrium does not mean breaking the matching between respiratory oxygen flow and cell oxygen demand.

It is noteworthy that this line of reasoning certainly acknowledges that in the transient from rest to the steady state of moderate exercise, there is an increase in lactate production from glycolysis. Indeed, it is widely recognized that lactate production goes up whenever the glycolytic activity increases (see e.g. Ferguson et al. 2018; Poole et al. 2021). As Poole et al. (2021) clearly point out, during moderate exercise, the equilibrium between lactate production and lactate removal is maintained unaltered, since both occur at the same rate, even within the same muscle fibre.

Notwithstanding, above a certain power, which may roughly correspond to the $50-60 \%$ of the maximal aerobic power, something different happens. According to Cerretelli et al. (1979), the $\tau$ of a mono-exponential $\dot{V} O_{2}$ increase at exercise onset becomes higher, the higher are the exercise intensity and $\dot{E}$. They showed that $\tau$ is linearly related to the amount of lactate that is accumulated in blood during the exercise transient, as described by

$\tau=\lambda[L a]_{e}+\tau_{0}$, where $[\mathrm{La}]_{e}$ is early lactate (net blood lactate accumulation during the exercise transient), $\lambda$ is a constant indicating how much $\tau$ increases per unit increase of lactate in blood during the exercise transient (di Prampero and Ferretti 1999), and $\tau_{0}$ is the time constant that would incur in the absence of early lactate accumulation. The constant $\lambda$ is inversely proportional to the steady-state $\dot{V} O_{2}$. According to Cerretelli et al. (1979), at a steady-state $\dot{V} O_{2}$ of 1.5 and $1.0 \mathrm{~L} \mathrm{~min}^{-1}$ (arm cranking mechanical powers of 125 and $75 \mathrm{~W}$ ), it is equal to 7.8 and $12.1 \mathrm{~s} \mathrm{mM}^{-1}$, respectively (Fig. 15).

These values allow computation of the oxygen deficit increase (in $\mathrm{mL} \mathrm{O}_{2} \mathrm{~kg}^{-1}$ ) per unit increase in $[\mathrm{La}]_{e}$ (in $\mathrm{mM}$ ), which corresponds to the energy equivalent of blood lactate accumulation. This result is equal to 2.19 and $2.89 \mathrm{~mL} \mathrm{~kg}^{-1} \mathrm{mM}^{-1}$ for the 125 and $75 \mathrm{~W}$ arm cranking power, respectively, the latter value agreeing particularly well with those reported in the literature (di Prampero 1981; di Prampero and Ferretti 1999). In turn, constant $\tau_{0}$ corresponds to the time constant describing Eq. (42). Accounting for the error due to extrapolation of a regression equation, and to possible yet small changes in blood oxygen stores, the values of $\tau_{0}$ obtained by Cerretelli et al. (1979) (24.5 and $29.7 \mathrm{~s}$ for the two equations reported in Fig. 15) correspond well to those obtained for the $\mathrm{PCr}$ decrease from ${ }^{31} \mathrm{P}-\mathrm{NMR}$ studies (Binzoni et al. 1992; di Prampero et al. 2003; Francescato et al. 2008, 2013; Rossiter et al. 1999, 2002). When early lactate appears during an exercise transient, the overall oxygen deficit becomes larger than the net alactic oxygen deficit, so that $\tau$ becomes higher than that of PCr drop. An energy gap appears, bridged by early lactate. In the next paragraph, we discuss the hypothesis that this energy gap is created by a progressive slowing of the $\dot{V}_{R} O_{2}$ kinetics as the exercise intensity goes up. Discussing this hypothesis, however, requires a preliminary analysis of the $\dot{Q}$ kinetics.

\section{Kinetics of cardiac output and oxygen delivery at exercise onset}

The first successful attempt at determining a kinetics of $\dot{Q}$ was not on a beat-by-beat basis (Cerretelli et al. 1966): this is perhaps why their kinetics of $\dot{Q}$ resulted slower than reported in subsequent studies. Even so, they realised that it was faster than that of $\dot{V}_{R} O_{2}$ and suggested that it may have a rapid initial increase, followed by a slower phase. They concluded that the discrepancy between the kinetics of $\dot{Q}$ and of $\dot{V}_{R} O_{2}$ demonstrates that the former is under neurogenic control. Davies et al. (1972) obtained similar results a few years later.

However, no kinetics of $\dot{Q}$ could be investigated on a beat-by-beat basis until the mid-1980s. Until then, only the kinetics of $f_{H}$ could be studied beat-by-beat in humans. The 
first $f_{H}$ data at exercise were obtained on rabbits in 1895 , using pulse pressure recordings through a cannula placed in the carotid artery (Johansson 1895). Krogh and Lindhard (1913b) were the first to describe a $f_{H}$ kinetics at exercise onset in humans. Thanks to the continuous developments of ECG recording techniques, hundreds of studies can be found in the literature on the $f_{H}$ kinetics during exercise. Among these, a paper by Fagraeus and Linnarsson (1976) has gained great importance, for its impact on further thinking. These authors investigated the effects of parasympathetic blockade on the $f_{H}$ kinetics at exercise onset. The rapid response of $f_{H}$, which they identified in control condition, disappeared under atropine. This is the strongest evidence obtained so far supporting the hypothesis of vagal tone withdrawal at exercise onset.

As soon as beat-by-beat techniques for the determination of $\dot{Q}$ became available, it soon became clear that also the kinetics of $\dot{Q}$ at exercise onset is very fast, much faster than stated by Cerretelli et al. (1966) and much faster than that of $\dot{V} O_{2}$ (Adams et al. 1987; Cummin et al. 1986; De Cort et al. 1991; Eriksen et al. 1990; Magder et al. 1987; Yoshida and Whipp 1994). Moreover, Cummin et al. (1986) highlighted a close correspondence between the rapid increase of $\dot{Q}$ and of ventilation at exercise onset, suggesting the hypothesis of an overall integrated response of the respiratory system in the exercise transient. None of those authors, however, proposed a model of the $\dot{Q}$ kinetics at exercise onset.

Lador et al. (2006) were the first to model the kinetics of $\dot{Q}$. In fact, they extended the domain of application of the dual exponential model of Barstow and Molé (1987) to describe also the kinetics of $\dot{Q}$. During moderate exercise, they obtained very low $\tau$ values not only for the initial rapid phase (phase I), but also for the second slower phase, or primary phase (phase II, $\tau_{0}$ equal to $2.1 \mathrm{~s}$ as compared to $15.4 \mathrm{~s}$ for $\dot{V} \mathrm{O}_{2}$ ). Since $\mathrm{C}_{a} \mathrm{O}_{2}$ does not vary in normoxic exercise, the kinetics of $\dot{Q}_{a} \mathrm{O}_{2}$ resulted practically superimposable to that of $\dot{Q}$. Application of a double-exponential model to the analysis of the kinetics of $\dot{Q}$, however, requires different interpretative hypotheses for each of the two phases of the $\dot{Q}$ response.

The rapid increase of $\dot{Q}$ in phase I is the consequence of the equally rapid increase of $f_{H}$ and of $Q_{s}$. For their interpretation of the rapid phase of the $f_{H}$ kinetics, Lador et al. (2006) relied on the vagal withdrawal hypothesis of Fagraeus and Linnarsson (1976). Coherently with this hypothesis, in hypoxia, when vagal activity is already reduced at rest, the amplitude of the $f_{H}$ increase in phase I was smaller than in normoxia (Lador et al. 2008). Further indirect support to this hypothesis was later provided by a study of heart rate variability at rest and exercise after parasympathetic blockade with atropine (Fontolliet et al. 2018). Recently, Fontolliet et al. (2021) demonstrated an almost complete suppression of phase I for $f_{H}$ under full vagal blockade with atropine.
Concerning the rapid increase in $Q_{s}$ (Faisal et al. 2009; Lador et al. 2006, 2008), a mechanical origin has been postulated. According to this hypothesis, a sudden increment in venous return may generate a rapid increase of $Q_{s}$ at the onset of exercise (Laughlin 1987; Leyk et al. 1994; Schneider et al. 2002; Sundblad et al. 2000). Similar mechanisms were proposed also to explain the $\dot{Q}$ increase in heart transplant recipients (Meyer et al. 1994) and patients carrying pacemakers for complete heart block (Nóbrega et al. 1997). Muscle pump action on venous blood flow by lower limb muscle contraction at exercise onset was identified as the possible mechanism enhancing venous return suddenly. Recently, the simultaneous determination of the kinetics of $f_{H}, Q_{s}$, and $\dot{Q}$ at exercise onset under various levels of exposure to lower body negative pressure provided strong support to this hypothesis (Fagoni et al. 2020). Yet, the mechanism whereby a phenomenon occurring in the right atrium (sudden increase in venous return) determines an immediate response by the left ventricle (sudden increase in $Q_{s}$ ) is still poorly understood. The interaction of several mechanisms has been evoked: the Frank-Starling mechanism, ventricular interdependence, with pulmonary circulation acting as a buffer mitigating the phenomenon (Chung et al. 1997; Naeije and Badagliacca 2017; Sundblad et al. 2000, 2014). A different track was taken by Elstad et al. (2009), who highlighted the role of muscle vasodilation and the subsequent $R_{p}$ fall in determining the rapid $Q_{s}$ response. According to them, the ensuing decrease in $\bar{P}$ would be the main determinant in the $Q_{s}$ dynamics. Whatever mechanism is operant, the putative cause of the phase I response of $Q_{s}$ may well differ from the neural mechanism acting on $f_{H}$, both coexisting and acting simultaneously to determine the rapid $\dot{Q}$ response (phase I) in healthy humans.

The phase I $\dot{Q}$ response is associated with an extremely fast, functionally instantaneous, phase I of the $\dot{V}_{R} O_{2}$ kinetics (Lador et al. 2006, 2008; Faisal et al. 2009). This implies an immediate upward translation of $\dot{V}_{R} O_{2}$, compatible with the observation (Cummin et al. 1986) of a close correspondence between the phase I increase of $\dot{Q}$ and ventilation at exercise onset. It provides also further support to the hypothesis (Wasserman et al. 1974; Whipp and Ward 1982) of the cardiac origin of the phase $\mathrm{I} \dot{V}_{R} O_{2}$ increase at exercise onset. Because of a delay between $\dot{V} O_{2}$ and $\dot{V}_{R} O_{2}$, we can assume that in the first seconds of exercise, the gas composition of mixed venous blood remains unchanged, so that $\mathrm{C}_{a} \mathrm{O}_{2}-\mathrm{C}_{\bar{v}} \mathrm{O}_{2}$ is the same as at rest (Barstow and Molé 1987; Weissman et al. 1982). If this is so, and were the $\dot{V} \mathrm{O}_{2}$ increase in phase I due only to an increase in $\dot{Q}$, especially in the pulmonary circulation, due to a sudden increase in venous return, application of the Fick principle to lung blood flow in phase I should provide a theoretical $\dot{V}_{R} O_{2}$ increase equal to the measured amplitude of the $\dot{V}_{R} O_{2}$ increase in the same phase. This analysis was performed by 
Lador et al. (2006), who found an average amplitude of the $\dot{Q}$ response in phase I equal to $4.3 \mathrm{~L} \mathrm{~min}^{-1}$. For an average resting $\mathrm{C}_{a} \mathrm{O}_{2}-\mathrm{C}_{\bar{v}} \mathrm{O}_{2}$ of $87 \mathrm{~mL} \mathrm{~L}^{-1}$, this would carry along a corresponding immediate $\dot{V} O_{2}$ increase of $374 \mathrm{~mL} \mathrm{~min}^{-1}$, which is very close to the observed amplitude of phase I for $\dot{V}_{R} O_{2}$, reported in the same study $\left(355 \pm 148 \mathrm{~mL} \mathrm{~min}^{-1}\right)$, as was to be demonstrated.

Lador et al. (2006) found also faster phase II $\tau$ of $\dot{Q}$ and $\dot{Q}_{a} \mathrm{O}_{2}$ than of $\dot{V} O_{2}$, with equal time delay. Thus, the amount of oxygen made available to the contracting muscle mass in phase II exceeded the oxygen demand. This caused a transient decrease of $\mathrm{C}_{a} \mathrm{O}_{2}-\mathrm{C}_{\bar{v}} \mathrm{O}_{2}$, with consequent transient increase of the oxygen stores in venous blood. They attributed the phase II increase in $\dot{Q}$ to the progressive activation of sympathetic heart stimulation. Under this condition, even though strictly speaking the performance of the respiratory system exceeds that of muscle metabolism, thus demonstrating an imperfect coupling of respiratory oxygen flow and muscle oxygen demand, the response of the respiratory system in coping with oxygen demand is anyway adequate. Thus, oxidative phosphorylation is accelerated at the necessary rate to follow the kinetics of activation of glycolysis: no early lactate accumulation occurs and Eq. (40) applies, as occurs in case of adequate coupling of respiration to metabolism.

\section{The $\dot{V} \mathrm{O}_{2}$ kinetics in hypoxia}

The primary component (phase II) of the $\dot{V} O_{2}$ kinetics in hypoxia shows a higher (slower) $\tau_{2}$ than in normoxia (Cleuziou et al. 2005; Engelen et al. 1996; Hughson and Kowalchuk 1995; Lador et al. 2008, 2013; Springer et al. 1991), as does the phase II kinetics of $\dot{Q}$ and $\dot{Q}_{a} O_{2}$ (Lador et al. 2008, 2013). Only one study, however, reported at the same time the values of early lactate accumulation (Lador et al. 2013): we therefore refer to that study for the present analysis.

The average cumulative volume of oxygen $\left(V_{L} O_{2}\right)$ moving across the alveolar-capillary barrier during a constant-load exercise is given by

$V_{L} O_{2}=\int_{0}^{t} d \dot{V}_{L} O_{2} d t$

The simultaneous changes in venous blood oxygen stores $\left(\Delta V_{v} O_{2}\right)$ occurring during the exercise transient are equal to (Barstow et al. 1990)

$\Delta V_{v} O_{2}=\left[\left(\frac{\dot{V} O_{2}}{\dot{Q}}\right)_{s s}-\left(\frac{\dot{V} O_{2}}{\dot{Q}}\right)_{r}\right] \cdot V_{v}$

where suffixes $s s$ and $r$ indicate the steady-state exercise and resting conditions, respectively, and $V_{v}$ is the venous blood volume, that Barstow et al. (1990) assumed invariant within the exercise transient and equal to $3 \mathrm{~L}$. On this basis, the results of Lador et al. (2013) yield a $\Delta V_{v} O_{2}$ equal to 260 and to $230 \mathrm{~mL}$ in normoxia and hypoxia, respectively. If we add these values to $V O_{2}$, we obtain the volume of oxygen extracted by the contracting muscles $\left(V_{2 M}\right)$ in the two conditions. In constant-power exercise, the muscular oxygen deficit $\left(D O_{2 M}\right)$ is equal to (di Prampero 1981; di Prampero and Ferretti 1999)

$D O_{2 M}=\dot{E} t-V O_{2 M}=\dot{V} O_{2} t-V O_{2 M}$,

where $t$ is the exercise time. Since the changes in blood oxygen stores are accounted for in the definition of $V_{2 M}$, we also have

$D O_{2 M}=D O_{2 A L}+D O_{2 L A}$,

where $D O_{2 L A}$ is the facultative component of $D O_{2 M}$, represented by $[L a]_{e}$.

As already pointed out, in homogeneous aerobic conditions, i.e., when $D O_{2 L A}$ is nil, $D O_{2 M}=D O_{2 A L}$ and the $\tau$ of muscle $\dot{V} O_{2}$ is equal to that of the mono-exponential PCr decrease (Binzoni and Cerretelli 1991), ranging between 20 and $25 \mathrm{~s}$. In the study of Lador et al. (2013), the homogeneous aerobic condition is represented by the experiments in normoxia: no increase in blood lactate was observed during the exercise transient. Therefore, the estimated $\tau$ of muscle $\dot{V} O_{2}$ kinetics corresponded well to that reported for muscle PCr kinetics. It is noteworthy that in this case, the phase II $\tau$ of $\dot{Q}$ and $\dot{Q}_{a} O_{2}$, and thus of $\dot{V}_{R} O_{2}$, were faster than that of $\dot{V} O_{2}$.

Things are not so in hypoxia, as long as $D O_{2 M}$ was larger than in normoxia and $[\mathrm{La}]_{e}$ was positive. Since in Lador et al. (2013), the applied mechanical power was the same in hypoxia as in normoxia, and so was the steady-state $\dot{V} O_{2}, D O_{2 A L}$ was necessarily equal in both conditions. Therefore, the increase in $D O_{2 M}$ in hypoxia was exclusively due to the increase in $D O_{2 L A}$. The energy provided by $D O_{2 L A}$ in hypoxia must be equal to the difference in $D O_{2 M}$ between hypoxia and normoxia. The ratio between $D O_{2 L A}$ and $[L a]_{e}(1.3 \mathrm{mM}$ in Lador et al. 2013), expressed per unit of body mass, turns out equal to $2.5 \mathrm{~mL} \mathrm{O}_{2} \mathrm{mM}^{-1} \mathrm{~kg}^{-1}$, a value fairly close to the range normally admitted for the energy equivalent of blood lactate accumulation (di Prampero 1981; di Prampero and Ferretti 1999). The energy balance fits. It is noteworthy, however, that in this case, the $\tau_{2}$ of $\dot{Q}$ and $\dot{Q}_{a} O_{2}$, and thus of $\dot{V}_{R} O_{2}$, were slower than that of $\dot{V} O_{2}$. 


\section{Matching things in the exercise transient}

\section{Equilibrium below the maximal lactate steady state}

To sum up, whenever the phase II kinetics of $\dot{Q}$ and $\dot{Q}_{a} O_{2}$, and thus of $\dot{V}_{R} O_{2}$, are faster than that of $\dot{V} O_{2}$, despite the rupture of the steady-state condition, a tight matching of $\dot{V}_{R} O_{2}$ to $\dot{V} O_{2}$ is maintained. However, as soon as the rate, at which $\dot{Q}$ and $\dot{Q}_{a} O_{2}$, and thus $\dot{V}_{R} O_{2}$, increase in an exercise transient, becomes as slow as or slower than the rate at which $\dot{V} O_{2}$ increases, then the tight coupling of $\dot{V}_{R} O_{2}$ to $\dot{V} \mathrm{O}_{2}$ is also broken, as is the tight coupling of respiration and metabolism. This occurs anytime the homogeneous aerobic condition is broken, around the so-called lactate threshold. In this case, although muscle blood flow may be able to cope with the energy demand, as suggested by some studies (Behnke et al. 2001; Grassi et al. 1996), the $\dot{V}_{\mathrm{R}} \mathrm{O}_{2}$ kinetics, and thus the muscle $\dot{\mathrm{V}} \mathrm{O}_{2}$ kinetics, become slower than the kinetics of PCr decrease, and the glycolytic pathway is accelerated at a faster rate than oxidative phosphorylation. When this occurs, pyruvate accumulates in contracting muscle fibres, especially type II muscle fibres, thus promoting muscle, then blood lactate accumulation. If the power, though higher than that ensuring $[L a]_{e}=0 \mathrm{mM}$, is low enough to allow the contracting muscle mass to operate in a heterogeneous aerobic condition (di Prampero and Ferretti 1999), a new equilibrium at a new apparent steady state is attained at a higher steady lactate concentration than that at rest. This lactate concentration may even be higher than $5 \mathrm{mM}$ (Ribeiro et al. 1986): its highest value was defined as the maximal lactate steady state (Beneke 2003). At higher workloads, the exercise can go on for a time (limit time, $t_{\mathrm{lim}}$ ) that is shorter the higher is the applied mechanical power.

The concept of maximal lactate steady state is strictly related to the concept of even or uneven, or homogeneous and heterogeneous, aerobic metabolism (Antonutto and di Prampero 1995; di Prampero and Ferretti 1999). During moderate exercise, all active muscle fibres (essentially type I slow fibres) operate in homogeneous aerobic conditions, which means that each fibre is able to complete the entire pathway from glucose to oxygen consumption within itself. In this case, the oxygen deficit consists only of its obligatory component, and muscle $\mathrm{PCr}$ kinetics is a mirror image of muscle $\dot{V} O_{2}$ kinetics.

Increasing power, and thus $\dot{E}$, carries along the recruitment of a larger number of motor units, and thus of muscle fibres. Also type IIb fibres, with lower mitochondrial volume and oxidative potential than aerobic type I fibres, need to be activated. The maximal $\dot{V} O_{2}$ of the former fibres is lower than that of the latter fibres, so that those fibres start accumulating lactate (hypoaerobic condition), while these fibres do not yet. This phenomenon may be accentuated by the progressive slowing of the kinetics of cardiovascular variables, which reduces the amount of oxygen relative to the energy demand that is provided to the contracting muscle mass during the exercise transient. Therefore, muscle lactate concentration increases. This tendency is counteracted, however, by the ability of type I fibres, which still have the possibility to increment their aerobic metabolism (hyperaerobic fibres), to take up lactate produced by adjacent type II fibres. This carries along a new equilibrium, whereby steady blood lactate concentration higher than at rest can be maintained. This condition, defined as unevenly aerobic condition (di Prampero and Ferretti 1999), is tantamount to the lactate shuttle concept (Brooks 1985, 2018; Brooks et al. 1999), is the source of $[L a]_{e}$, and allows reestablishment of an equilibrium between $\dot{V}_{\mathrm{R}} \mathrm{O}_{2}$ and $\dot{\mathrm{V}} \mathrm{O}_{2}$. Nevertheless, this equilibrium is fragile, precarious. The appearance of the so-called "slow component" of the $\dot{V}_{R} O_{2}$ kinetics (Camus et al. 1988; Paterson and Whipp 1991; Poole et al. 1988; Whipp and Wasserman 1972) pertains to this exercise domain. For a more detailed discussion of the physiological and energetic meaning of the slow component, we address the reader to more specific references (Jones et al. 2011; Poole and Jones 2012; Poole et al. 1994).

\section{Disequilibrium above maximal lactate steady state or critical power}

If we further increase the exercise intensity, no form of equilibrium can be attained anymore. Both the metabolic rate of type I fibres and the lactate production by type IIb fibres increase. Although the power is still below the individual maximal aerobic power, muscle and blood lactate concentrations keep increasing with time. Muscle $\mathrm{pH}$ drops. Type IIb fibres fatigue and their efficiency decrease. Type I fibres become unable to oxidize also the lactate produced by type IIb fibres. The amplitude of the slow component becomes larger, $\dot{V}_{R} O_{2}$ increases until it reaches its maximum and exercise is then terminated (Poole et al. 1988). At even higher powers, also type I fibres attain their maximal $\dot{V} \mathrm{O}_{2}$ : we enter the supramaximal exercise domain, all active muscle fibres produce lactate, the rate of blood lactate accumulation, and thus, the term $b \dot{L a}$ of Eq. (2) (anaerobic lactic power, as defined by Margaria et al. 1963), becomes higher and higher as the applied mechanical power is further increased. Blood homeostasis is under attack, the massive stimulation of central chemoreceptors determines a strong hyperventilation, but the rate of blood lactate accumulation overrides the capacity of controlling $\mathrm{pH}$. Muscle $\mathrm{pH}$ drops dramatically and glycolysis is inhibited: exercise must be stopped. When the maximal lactic power has been attained (Margaria et al. 1964), this occurs in less than 1 min after the exercise onset. 


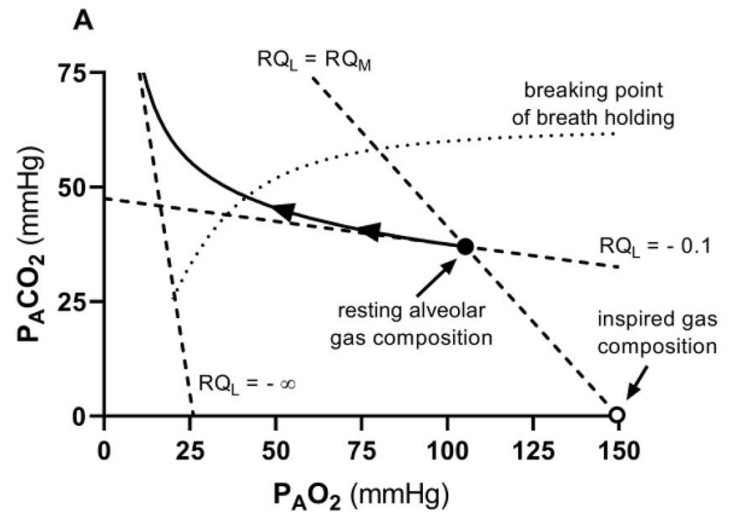

Fig. 16 A Graphical representation of the hypoventilation loop model of the alveolar pathway during breath-holding (continuous curve) in air. Dashed lines represent the constructing lines, which are isopleths of the indicated lung respiratory quotient $\left(R Q_{L}\right)$. The dotted curve represents the conventional breaking point of breath-holding. Black arrowheads indicate the progressive changes of alveolar gas composition starting from the resting alveolar gas composition preceding breath-hold (black dot). $R Q_{M}$ metabolic respiratory quotient

The relationship between $t_{\text {lim }}$ and power in this intense exercise domain is hyperbolic, as described by the critical power model (Jones et al. 2010; Monod and Scherrer 1965), and more precisely by the three-parameter critical power model (Morton 1996), which includes also extreme exercise intensities (Vinetti et al. 2019), up to the maximal lactic power. The critical power models clearly indicate that the new equilibrium here is not achievable, affecting maximal exercise duration.

The homeostatic equilibrium of the milieu intérieur, totally upset in supramaximal exercise, is re-established in the recovery phase after the end of exercise, including the return to a tight match between respiration and metabolism. The timing of functional recovery, which was precisely described already by Margaria et al. (1933), depends on the kinetics of lactate washout by oxidation to pyruvate, which then feeds the Krebs' cycle, in the muscle fibres as well as in other organs. The kinetics of lactate removal is accelerated if moderate exercise is performed in the recovery period (Belcastro and Bonen 1975; Gisolfi et al. 1966; Reaburn and McKinnon 1990).

\section{Breaking the equilibrium: breath-holding}

Breath-holding implies a sudden shift to $\dot{V}_{A}=0 \mathrm{~L} \mathrm{~min}{ }^{-1}$, so that the $\dot{V}_{A} / \dot{V}_{R} C O_{2}$ immediately jumps from -21.6 to 0 . This represents a dramatic rupture of the homeostatic equilibria in the respiratory system, determining abrupt unsteady state and uncoupling of respiration from metabolism within seconds. Since no gas exchange between ambient air and alveolar air occurs anymore, $P_{A} O_{2}$ and $P_{a} O_{2}$

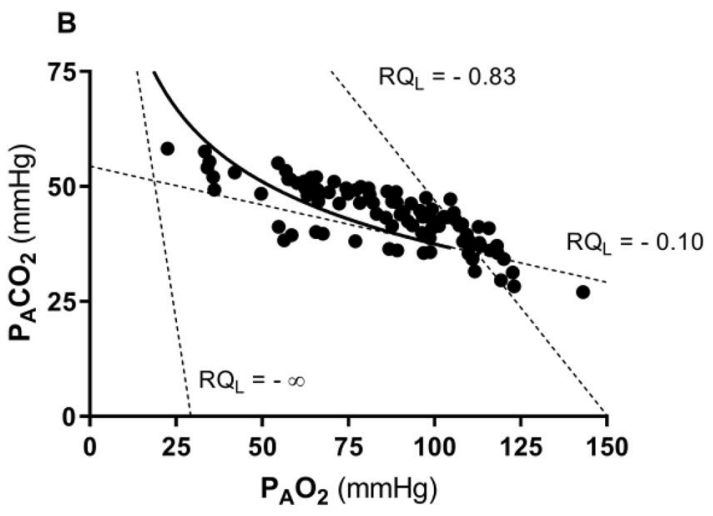

(at rest, - 0.83). B Experimental data of alveolar gas composition at the end of resting breath-holds of increasing duration up to the maximal, in air. The continuous curve represents the fitting function of the hypoventilation loop model. Dashed lines represent the constructing straight lines of the hypoventilation loop. $\mathrm{P}_{A} \mathrm{O}_{2}$ and $\mathrm{P}_{\mathrm{A}} \mathrm{CO}_{2}$ alveolar partial pressures of oxygen and carbon dioxide, respectively [Modified after Taboni et al. (2020)]

fall impressively, whereas $P_{A} \mathrm{CO}_{2}$ does not increase that much, only because most of the carbon dioxide produced by metabolism is accumulated in tissues. Yet the combination of hypoxia and hypercapnia leads rapidly to strong respiratory stimuli, which cause first diaphragmatic contractions, then the interruption of breath-holding. The catastrophe of the milieu interrieur is so dramatic, that the interruption of breath-holding at rest, even if performed starting from a lung volume equal to the total lung capacity, normally intervenes within 3 min (Ferretti 2001; Lindholm and Lundgren 2009). And the uncoupling of respiration and metabolism, accentuated by the appearance of a diving response even in humans, is not the most serious event under these circumstances, although it is the subject of this review. For a more general overview of the physiology of breath-holding and breath-hold diving, we address the reader to other, more specific, review articles (Bain et al. 2018; Elia et al. 2021c; Ferretti 2001; Fitz-Clarke 2018; Lindholm and Lundgren 2009; Pendergast and Lundgren 2009).

Serial analyses of alveolar gas composition at the end of breath-holds of increasing duration have led to set the limits of voluntary breath-holding at the boundaries of the anoxic collapse and of the hypercapnic narcosis regions on an $\mathrm{O}_{2}-\mathrm{CO}_{2}$ diagram (Ferretti et al. 1991; Lin et al. 1974). The attainment of these limits follows alveolar pathways during breath-holding, which have been precisely analysed over 5 decades ago (Agostoni 1963; Fagoni et al. 2017; Ferretti et al. 1991; Lanphier and Rahn 1963; Lin et al. 1974; Lindholm and Lundgren 2006; Otis et al. 1948; Taboni et al. 2019).

Oxygen has a much lower solubility in blood and cells than carbon dioxide (respectively, 0.0214 and $0.515 \mathrm{~mL}$ of 
gas per millilitre of human plasma at sea level at $37{ }^{\circ} \mathrm{C}$; Christmas and Bassingthwaighte 2017), so that carbon dioxide storage capacity is some 25 times larger than oxygen storage capacity. Blood and lungs are mostly involved, with small capacity and thus fast equilibrium time constants (Farhi and Rahn 1960; Linér and Linnarsson 1994). Thus, the amount of carbon dioxide that goes into rapidly exchanging tissues at the beginning of a hypoventilation is by far larger than the amount of oxygen that moves out of the same tissues, before a new equilibrium is attained.

These are the reasons why the patterns followed by alveolar gases in the unsteady state at the beginning of hypoventilation reproduce what we call a hypoventilation loop (Farhi and Rahn 1955; Rahn and Fenn 1955). The curvilinear segment representing a hypoventilation loop on the $\mathrm{O}_{2}-\mathrm{CO}_{2}$ diagram is concave, starts and ends on the prevailing steady-state $R Q_{L}$ line, and follows a pattern moving leftward to it. This segment is larger, the closer to zero is the $\dot{V}_{A} / \dot{V}_{R} C O_{2}$ during hypoventilation.

Notwithstanding, validation of the hypoventilation loop concept has been rarely performed. The most complete study is still that by Rahn and Otis (1949), who induced acute alveolar hypoventilation by artificially increasing the anatomical dead space by adding a hose between the mouth and the breathing valve. They found that the alveolar pathway initially moved leftward and then upward on the $\mathrm{O}_{2}-\mathrm{CO}_{2}$ diagram. Farhi and Rahn (1955) later proposed that the alveolar pathway of ventilatory unsteady states can be described by exponential functions.

Taboni et al. (2020) treated the alveolar gas pathways during breath-holding as an extreme hypoventilation loop. The theoretical loop is shown in Fig. 16a. The experimental data coincide with the model (Fig. 16b). The hypoventilation loop in ambient air is characterised by two segments. One is almost horizontal, depicting a large decrease in $\mathrm{P}_{A} \mathrm{O}_{2}$ accompanied by a minimal increase in $\mathrm{P}_{A} \mathrm{CO}_{2}$; the second segment bends upward, being characterised by a smaller decrease in $\mathrm{P}_{A} \mathrm{O}_{2}$ and a larger increase in $\mathrm{P}_{A} \mathrm{CO}_{2}$. The flat segment reflects the pressure gradient generated by the sudden fall of the $\dot{V}_{A} / \dot{V}_{R} C O_{2}$, which carries along the patterns of carbon dioxide storage in tissues (Farhi and Rahn 1960), while the oxygen stores are slowly emptied. The oxygen outflow from tissues does not correct for the oxygen transfer to lung capillaries in the absence of oxygen inflow from ambient air, whence the large fall of $P_{A} O_{2}$. This implies an extremely high $R Q_{L}$, totally dissociated from $R Q_{M}$, as reported in several studies (Ferretti et al. 1991; Lin et al. 1974; Otis et al. 1948; Taboni et al. 2020). In a few cases, a positive $R Q_{L}$ was even found, suggesting reversal of carbon dioxide flux at the alveolar-capillary barrier (Ferretti et al. 1991; Lindholm and Linnarsson 2002). No correspondence between pulmonary gas exchange and tissue metabolism exists anymore.
As long as the pressure gradient sustaining it goes down, the gas flows between tissues and alveoli (or vice versa) decreases exponentially, tending toward a new equilibrium. As this occurs, the hypoventilation loop bends upward. As the equilibrium between alveoli and rapidly exchanging tissues has been attained, the $\mathrm{P}_{A} \mathrm{O}_{2}$ has become so low, that the pressure gradient sustaining oxygen flow across the alveolar-capillary barrier tends to zero. At the same time, the carbon dioxide produced by metabolism is entirely added to the alveoli, so that $R Q_{L}$ tends toward infinity, although the $\dot{V}_{A} / \dot{V}_{R} C O_{2}$ remains at 0 . We attain the second trait of the hypoventilation loop.

In this condition, $\dot{V}_{R} O_{2}$ might still partly be sustained by changes in blood oxygen stores, through an increase in oxygen delivery associated with diaphragmatic contractions (Palada et al. 2008) and incurring arterial and venous haemoglobin desaturation (Lindholm et al. 1999, 2002), and by changes in muscle oxygen stores, which may be enhanced in divers (Elia et al. 2019). Blood and muscle oxygen stores may be improved by apnoea training (Elia et al. 2021a) and eventually by splenic contraction (Elia et al. 2021b).

Notwithstanding, this second part of the extreme hypoventilation loop cannot undergo experimental validation, so that it is deemed to remain a theoretical concept, because alveolar gas composition would go beyond that compatible with consciousness, if not with life. A maximal breath-hold is inevitably interrupted at an alveolar gas composition around that of the conventional breaking point curve (Lin et al. 1974). This allows predictions of the alveolar gas composition at the end of maximal breath-holds, independent of their duration, as it should correspond to the intersection of the experimental segment of the hypoventilation loop and the conventional breaking point curve, which also was modelled by fitting experimental data (Taboni et al. 2019).

During exercise apnoea, the patterns followed by alveolar gas composition are the same as at rest (Taboni et al. 2020). However, the time leading to the breaking point of apnoea is much shorter than at rest, because $\dot{E}$ is higher, as is $\dot{Q}$, so that the patterns of the cardiovascular responses to apnoeas are altered, and the rates of change of $\mathrm{P}_{A} \mathrm{O}_{2}$ and $\mathrm{P}_{A} \mathrm{CO}_{2}$ are increased (Lindholm et al. 2002; Sivieri et al. 2015; Taboni et al. 2018; Tocco et al. 2012) with respect to those demonstrated at rest (Costalat et al. 2013; Fagoni et al. 2015; Perini et al. 2008, 2010; Tocco et al. 2013).

During dives, alveolar gas composition is influenced also by changes in pressure and thus by the reduction of lung volume due to Boyle's law (Ferretti 2001) and by complete pulmonary shunt at extreme depths (Fitz-Clarke 2007, 2009). The continuously changing external pressure during diving affects the dynamics of oxygen and carbon dioxide flows from and into the rapidly exchanging tissues, as compared with surface breath-holding (Linér and Linnarsson 1994). A hint of what may happen indeed during a dive has been 


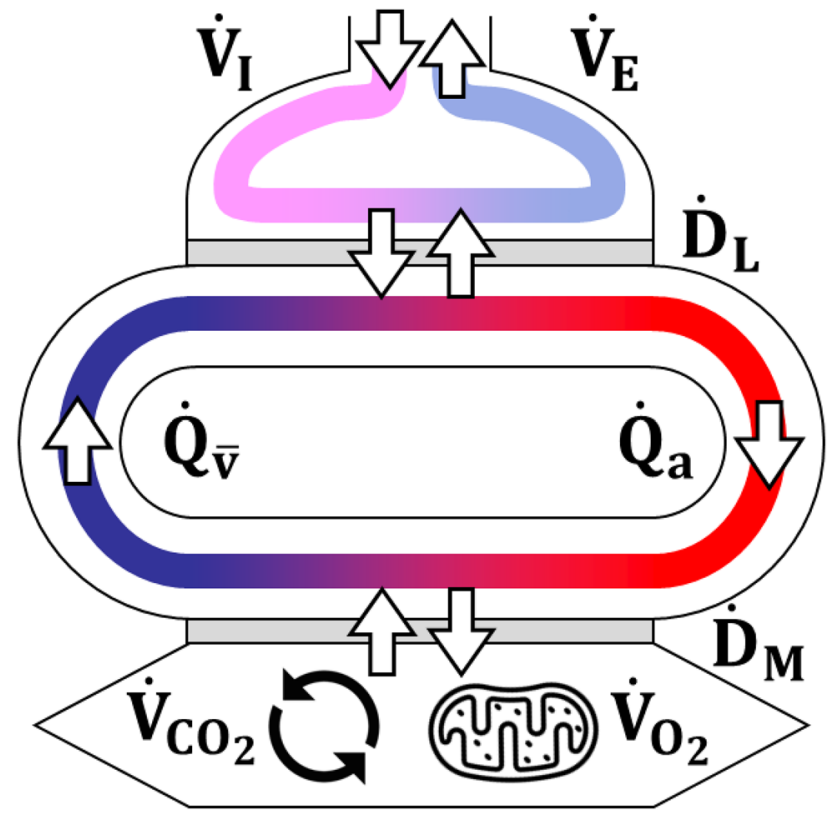

Fig. 17 Schematic representation, in the form of a visual abstract, of the flow of oxygen and carbon dioxide along the respiratory system, from ambient air up to the actual site of chemical production (Krebs cycle) or consumption (mitochondria). Each white arrow represents a potential source of deviation from the steady state of the integrated flows. The oxygen or the carbon dioxide flows at a certain level along the system correspond to the difference between two opposite white arrows. $\dot{V}_{I}$ inspired gas flow, $\dot{V}_{E}$ expired gas flow, $\dot{D}_{L}$ lung diffusive flow of a gas, $\dot{Q}_{a}$ arterial flow of a gas, $\dot{Q}_{\bar{v}}$ venous flow of a gas, $\dot{D}_{M}$ muscular diffusive flow of a gas, $\dot{\mathrm{V}} \mathrm{O}_{2}$ oxygen consumption, $\dot{\mathrm{V}} \mathrm{CO}_{2}$ carbon dioxide production

provided by the analysis of $\mathrm{P}_{a} \mathrm{O}_{2}$ and $\mathrm{P}_{a} \mathrm{CO}_{2}$ during dives in simulated and real diving conditions (Bosco et al. 2020; Muth et al. 2003).

To sum up, breath-holding disrupts all equilibria in the respiratory system and no coupling of respiration to metabolism at all is possible. Whereas the cells try to cope with their energy demand, all regulations of the respiratory system are turned upside down. This being so, the only possibility of restoring the homeostatic equilibria is to interrupt apnoea. This occurs as soon as the conventional breaking point has been attained.

\section{Conclusions}

In this review, we have outlined the conditions and constraints wherein a tight coupling of the respiratory system, meant in its holistic form (Taylor and Weibel 1981), and the cell (mostly muscle fibres during exercise) energy demand is attained. These conditions have been summarized in the form of a colour visual abstract in Fig. 17. The analysis of the steady-state condition has shown that the homeostatic equilibrium is centred on an arterial $\mathrm{pH}$ of
7.4 and a $P_{a} C O_{2}=P_{A} C O_{2}$ of $40 \mathrm{mmHg}$, with a $\dot{V}_{A} / \dot{V}_{R} C O_{2}$ of - 21.6. Several potential equilibria among the pertinent variables ensuring gas transfer along the respiratory system are compatible with the aforementioned homeostatic equilibrium, and they are actually exploited for instance at exercise, thus allowing adjustment of oxygen flow to oxygen demand.

These equilibria are broken in unsteady state. This may or may not break also the homeostatic equilibrium and the coupling of respiration and metabolism. This is not the case during the transient phase from rest to moderate exercise, when the response of the respiratory system is faster than that of the activation of the aerobic metabolic pathway. As the exercise intensity becomes higher, the response of the respiratory system gets slower and $[L a]_{e}$ appears: in this case, the coupling between respiration and metabolism is lost, but it is re-established as soon as, at steady state, lactate concentration stabilizes at a level that is higher than resting. However, if the increase in lactate concentration is such that the rapid blood buffering systems are unable to maintain arterial blood $\mathrm{pH}$ at 7.4, a slight hyperventilation, implying lower $\dot{V}_{A} / \dot{V}_{R} C O_{2}$ and $\mathrm{P}_{a} \mathrm{CO}_{2}$ below $40 \mathrm{mmHg}$.

In the severe exercise domain, above the critical power, the uncoupling cannot be recovered, anaerobic lactic metabolism provides energy beside and above the aerobic metabolism for the entire exercise duration, lactate concentration goes up, and arterial $\mathrm{pH}$ falls continuously, as does the $\mathrm{pH}$ of interstitial fluid. The absolute $\dot{V}_{A} / \dot{V}_{R} C_{2}$ keeps decreasing below -21.6 , because of the ensuing hyperventilation, trying to restore $\mathrm{pH}$; however, lactate keeps being accumulated in muscle and blood, at a rate that is higher, the higher is the exercise intensity. When this occurs, exhaustion is imminent.

The most catastrophic rupture of the homeostatic equilibrium occurs during breath-holding, because of the forced interruption of the oxygen flow from ambient air to mitochondria along the respiratory system, which is blocked and upset. No coupling at all is possible between respiration and metabolism in this case.

Acknowledgements This work is supported by Swiss National Science Foundation grant 32003B_179448 to Guido Ferretti. This review is dedicated to the memory of Rodolfo Margaria, Johannes Piiper, and Hermann Rahn and their unequalled mastership.

Author contributions GF conceived and structured the review; PdP drafted and GF completed the historical section; NF and GF drafted the steady-state sections; GF and GV drafted the exercise transient sections; NF and AT drafted the breath-hold section; GF and PdP put the various sections together in a coherent frame, to form a complete draft; GV and AT checked formal coherence of equations; GF and AT prepared figures; all authors read and commented the complete draft; on this basis, GF prepared the final version; all authors read and approved the final version. 


\section{Declarations}

Conflict of interest The authors declare no conflict of interest.

Open Access This article is licensed under a Creative Commons Attribution 4.0 International License, which permits use, sharing, adaptation, distribution and reproduction in any medium or format, as long as you give appropriate credit to the original author(s) and the source, provide a link to the Creative Commons licence, and indicate if changes were made. The images or other third party material in this article are included in the article's Creative Commons licence, unless indicated otherwise in a credit line to the material. If material is not included in the article's Creative Commons licence and your intended use is not permitted by statutory regulation or exceeds the permitted use, you will need to obtain permission directly from the copyright holder. To view a copy of this licence, visit http://creativecommons.org/licenses/by/4.0/.

\section{References}

Abovsky M, Lanir Y, Nevo E (1996) Tethering affects the mechanics of coronary capillaries. J Biomech 29:597-607. https://doi.org/ 10.1016/0021-9290(95)00122-0

Adair GS (1925) The hemoglobin system VI. The oxygen dissociation curve of hemoglobin. J Biol Chem 63:529-545. https://doi.org/ 10.1016/S0021-9258(18)85018-9

Adami A, Fagoni N, Ferretti G (2014) The $\dot{Q}-\dot{V} O_{2}$ diagram: an analytical interpretation of oxygen transport in arterial blood during exercise in humans. Respir Physiol Neurobiol 193:55-61. https:// doi.org/10.1016/j.resp.2014.01.007

Adams L, Guz A, Innes JA, Murphy K (1987) The early circulatory and ventilatory response to voluntary and electrically induced exercise in man. J Physiol 383:19-30. https://doi.org/10.1113/ jphysiol.1987.sp016393

Agostoni E (1963) Diaphragm activity during breath holding: factors related to its onset. J Appl Physiol 18:30-36. https://doi.org/10. 1152/jappl.1963.18.1.30

Anchisi S, Moia C, Ferretti G (2001) Oxygen delivery and oxygen return in humans exercising in acute normobaric hypoxia. Pflüg Arch 442:443-450. https://doi.org/10.1007/s004240100553

Andersson B (1933) Über Co-Zymaseaktivierung einiger Dehydrogenasen. Z Physiol Chem 217:186-190

Antonutto G, di Prampero PE (1995) The concept of lactate threshold: a short review. Int J Sports Med Phys Fit 35:6-12

Armstrong RB, Delp MD, Goljan MF, Laughlin MH (1987) Distribution of blood flow in muscles of miniature swine during exercise. J Appl Physiol 62:1285-1298. https://doi.org/10.1152/jappl. 1987.62.3.1285

Asmussen E (1973) Ventilation at transition from rest to exercise. Acta Physiol Scand 89:68-78. https://doi.org/10.1111/j.1748-1716. 1973.tb05498.x

Asmussen E, Nielsen M (1948) Studies on the initial changes in respiration at the transition from rest to work and from work to rest. Acta Physiol Scand 16:270-285. https://doi.org/10.1111/j. 1748-1716.1948.tb00544.x

Asmussen E, Nielsen M (1952) The cardiac output in rest and work determined simultaneously by the acetylene and the dye injection methods. Acta Physiol Scand 27:217-230. https://doi.org/ 10.1111/j.1748-1716.1953.tb00937.x

Åstrand PO, Christensen EH (1963) Hyperpnoea of exercise. In: Cunningham DJC, Lloyd BB (eds) The regulation of human respiration. Blackwell, Oxford, pp 515-524
Åstrand PO, Cuddy TE, Saltin B, Stenberg J (1964) Cardiac output during submaximal and maximal work. J Appl Physiol 19:258-264. https://doi.org/10.1152/jappl.1964.19.2.268

Åstrand PO, Rodahl K, Dahl HA, Strømme SB (2003) Textbook of work physiology. Physiological bases of exercise, 4th edn. Human Kinetics, Champaign

Atwater WO (1904) Neue Versuche über Stoff- und Kraftwechsel im menschlichen Körper. Ergeb Physiol 3:497-622

Auchincloss JH Jr, Gilbert R, Baule GH (1966) Effect of ventilation on $\mathrm{O}_{2}$ transfer during early exercise. J Appl Physiol 21:810-818. https://doi.org/10.1152/jappl.1966.21.3.810

Bain AR, Drvis I, Dujic Z, MacLeod DB, Ainslie PN (2018) Physiology of static breath holding in elite apneist. Exp Physiol 103:635-651. https://doi.org/10.1113/EP086269

Bake B, Bjure G, Widimsky J (1968) The effect of sitting and graded exercise on the distribution of pulmonary blood flow in healthy subjects studied with the ${ }^{133}$ Xenon technique. Scand J Clin Lab Invest 22:99-106. https://doi.org/10.3109/0036551680 9160952

Barstow TJ, Molé PA (1987) Simulation of pulmonary $\mathrm{O}_{2}$ uptake during exercise transients in humans. J Appl Physiol 63:2253-2261. https://doi.org/10.1152/jappl.1987.63.6.2253

Barstow TJ, Lamarra N, Whipp BJ (1990) Modulation of muscle and pulmonary $\mathrm{O}_{2}$ uptakes by circulatory dynamics during exercise. J Appl Physiol 68:979-989. https://doi.org/10.1152/jappl.1990. 68.3.979

Bates DV, Boucot NG, Dormer AE (1955) The pulmonary diffusing capacity in normal subjects. J Physiol 129:237-252. https://doi. org/10.1113/jphysiol.1955.sp005350

Baumgartner WA Jr, Jaryszak EM, Peterson AJ, Presson RG Jr, Wagner WW Jr (2003) Heterogeneous recruitment among adjoining alveoli. J Appl Physiol 95:469-476. https://doi.org/10.1152/jappl physiol.01115.2002

Beck KC, Johnson BD, Olson TP, Wilson TA (2012) Ventilation-perfusion distribution in normal subjects. J Appl Physiol 113:872877. https://doi.org/10.1152/japplphysiol.00163.2012

Behnke BJ, Kindig CA, Musch TI, Koga S, Poole DC (2001) Dynamics of microvascular oxygen pressure across the rest-exercise transition in rat skeletal muscle. Respir Physiol 126:53-63. https://doi. org/10.1016/S0034-5687(01)00195-5

Behnke BJ, Barstow TJ, Kindig CA, McDonough P, Musch TI, Poole DC (2002) Dynamics of oxygen uptake following exercise onset in rat skeletal muscle. Respir Physiol Neurobiol 133:229-239. https://doi.org/10.1016/s1569-9048(02)00183-0

Beinert H (1963) Acyl coenzyme A dehydrogenases. In: Boyer PD, Lardy H, Myrbäck K (eds) The enzymes, chapter 17, vol VII. Academic Press Inc., New York, pp 447-476

Belcastro AN, Bonen A (1975) Lactic acid removal rates during controlled and uncontrolled recovery exercise. J Appl Physiol 39:932-936. https://doi.org/10.1152/jappl.1975.39.6.932

Beneke R (2003) Maximal lactate steady state concentration (MLSS): experimental and modelling approaches. Eur J Appl Physiol 88:361-369. https://doi.org/10.1007/s00421-002-0713-2

Bernard C (1859) Leçons sur les propriétés physiologiques et les altérations physiologiques des liquides de l'organisme. Baillière, Paris

Bernard CC (1865) Introduction à l'étude de la médecine expérimentale. Baillière, Paris

Bertinieri G, Di Rienzo M, Cavallazzi A, Ferrari AU, Pedotti A, Mancia G (1988) Evaluation of baroreceptor reflex by blood pressure monitoring in unanesthetized cats. Am J Physiol Heart Circ Physiol 254:H377-H383. https://doi.org/10.1152/ajpheart.1988. 254.2.H377

Bevegård BS, Shepherd JT (1966) Circulatory effects of stimulating the carotid arterial stretch receptors in man at rest and during exercise. J Clin Invest 45:132-142. https://doi.org/10.1172/JCI10 5317 
Bevegård S, Freyschuss U, Strandell T (1966) Circulatory adaptation to arm and leg exercise in supine and sitting position. J Appl Physiol 21:37-46. https://doi.org/10.1152/jappl.1966.21.1.37

Binzoni T, Cerretelli P (1991) Muscle ${ }^{31}$ P-NMR in humans: estimate of bias and qualitative assessment of ATPase activity. J Appl Physiol 71:1700-1704. https://doi.org/10.1152/jappl.1991.71.5.1700

Binzoni T, Ferretti G, Schenker K, Cerretelli P (1992) Phosphocreatine hydrolysis by ${ }^{31} \mathrm{P}-\mathrm{NMR}$ at the onset of constant-load exercise in humans. J Appl Physiol 73:1643-1649. https://doi.org/10.1152/ jappl.1992.73.4.1644

Binzoni T, Hiltbrand E, Yano T, Cerretelli P (1997) Step vs. progressive exercise: the kinetics of phosphocreatine hydrolysis in human muscle. Acta Physiol Scand 159:209-215. https://doi. org/10.1046/j.1365-201X.1997.577354000.x

Bock AV, Vancaulert C, Dill DB, Fölling A, Hurxthal LM (1928) Studies in muscular activity. IV. The steady state and the respiratory quotient during work. J Physiol 66:162-174. https://doi.org/10. 1113/jphysiol.1928.sp002515

Bohr C (1891) Über Die Lungenathmung. Skand Arch Physiol 2:236238. https://doi.org/10.1111/j.1748-1716.1891.tb00581.x

Borg TK, Caulfield JB (1980) Morphology of connective tissue in skeletal muscle. Tissue Cell 12:197-207. https://doi.org/10.1016/ 0040-8166(80)90061-0

Borland C, Mist C, Zammit M, Vuylsteke A (2001) Steady-state measurement of $\mathrm{NO}$ and $\mathrm{CO}$ lung diffusing capacity on moderate exercise in men. J Appl Physiol 90:538-544. https://doi.org/10.1152/ jappl.2001.90.2.538

Bosco G, Paganini M, Rizzato A, Martani L, Garetto G, Lion J, Camporesi EM, Moon RE (2020) Arterial blood gases in divers at surface after prolonged breath-hold. Eur J Appl Physiol 120:505512. https://doi.org/10.1007/s00421-019-04296-2

Bringard A, Adami A, Fagoni N, Fontolliet T, Lador F, Moia C, Tam E, Ferretti G (2017) Dynamics of the RR-interval versus blood pressure relationship at exercise onset in humans. Eur J Appl Physiol 117:619-630. https://doi.org/10.1007/s00421-017-3564-6

Bristow JD, Honour AJ, Pickering GW, Sleight P, Smyth HS (1969) Diminished baroreflex sensitivity in highblood pressure. Circulation 39:48-54. https://doi.org/10.1161/01.cir.39.1.48

Bristow JD, Brown EB Jr, Cunningham DJ, Goode RC, Howson MG, Sleight $P$ (1971) The effects of hypercapnia, hypoxia and ventilation on the baroreflex regulation of the pulse interval. J Physiol 216:281-302. https://doi.org/10.1113/jphysiol.1971.sp009525

Brognara F, Felippe ISA, Salgado HC, Paton JFR (2021) Autonomic innervation of the carotid body as a determinant of its sensitivity: implications for cardiovascular physiology and pathology. Cardiovasc Res 117:1015-1032. https://doi.org/10.1093/cvr/cvaa250

Brooks G (1985) Anaerobic threshold: review of the concept and directions for future research. Med Sci Sports Exerc 17:22-31

Brooks GA (2000) Intra-and extra-cellular lactate shuttles. Med Sci Sports Exerc 32:790-799. https://doi.org/10.1097/00005768200004000-00011

Brooks GA (2012) Bioenergetics of exercising humans. Compr Physiol 2:537-562. https://doi.org/10.1002/cphy.c110007

Brooks GA (2018) The science and translation of lactate shuttle theory. Cell Metab 27:757-785. https://doi.org/10.1016/j.cmet.2018.03. 008

Brooks G, Dubouchaud H, Brown H, Sicurello JP, Butz CE (1999) Role of mitochondrial lactate dehydrogenase and lactate oxidation in the intracellulare lactate shuttle. Proc Natl Acad Sci 96:11291134. https://doi.org/10.1073/pnas.96.3.1129

Bryan AC, Bentivoglio LG, Beerel F, McLeish H, Zidulka A, Bates DV (1964) Factors affecting regional distribution of ventilation and perfusion in the lung. J Appl Physiol 19:395-402. https:// doi.org/10.1152/jappl.1964.19.3.395

Burnham KJ, Arai TJ, Dubowitz DJ, Henderson AC, Holverda S, Buxton RB, Prisk GK, Hopkins SR (2009) Pulmonary perfusion heterogeneity is increased by sustained, heavy exercise in humans. J Appl Physiol 107:1559-1568. https://doi.org/10.1152/ japplphysiol.00491.2009

Calbet JA (2000) Oxygen tension and content in the regulation of limb blood flow. Acta Physiol Scand 168:465-472. https://doi.org/10. 1046/j.1365-201x.2000.00698.x

Calbet JAL, Lundby C, Koskolou M, Boushel R (2006) Importance of haemoglobin concentration to exercise: acute manipulations. Respir Physiol Neurobiol 151:132-140. https://doi.org/10.1016/j. resp.2006.01.014

Camus G, Atchou G, Bruckner JC, Giezendanner D, di Prampero PE (1988) Slow upward drift of $\mathrm{VO}_{2}$ during constant-load cycling in untrained subjects. Eur J Appl Physiol 58:197-202. https://doi. org/10.1007/BF00636626

Cano I, Roca J, Wagner PD (2015) Effects of lung ventilation-perfusion and muscle metabolism-perfusion heterogeneities on maximal $\mathrm{O}_{2}$ transport and utilization. J Physiol 593:1841-1856. https://doi. org/10.1113/jphysiol.2014.286492

Capelli C, Cautero M, di Prampero PE (2001) New perspectives in breath-by-breath determination of alveolar gas exchange in humans. Pflüg Arch 441:566-577. https://doi.org/10.1007/s0042 40000429

Carlson FD, Siger A (1959) The creatine phosphoryltransfer reaction in iodoacetate-poisoned muscle. J Gen Physiol 43:301-313. https:// doi.org/10.1152/10.1085/jgp.43.2.301

Casey DP, Joyner MJ (2011) Local control of skeletal muscle blood flow during exercise: influence of available oxygen. J Appl Physiol 111:1527-1538. https://doi.org/10.1152/japplphysiol. 00895.2011

Caulfield JB, Tao SB, Nachtigal M (1985) Ventricular collagen matrix and alterations. Adv Myocardiol 5:257-269. https://doi.org/10. 1007/978-1-4757-1287-2_20

Cautero M, Beltrami AP, di Prampero PE, Capelli C (2002) Breathby-breath alveolar $\mathrm{O}_{2}$ transfer at the onset of step exercise in humans. Eur J Appl Physiol 88:203-213. https://doi.org/10.1007/ s00421-002-0671-8

Cautero M, di Prampero PE, Tam E, Capelli C (2005) Alveolar oxygen uptake kinetics with step, impulse and ramp exercise in humans. Eur J Appl Physiol 95:474-485. https://doi.org/10.1007/ s00421-005-0038-Z

Celsing F, Nyström J, Pihlstedt P, Werner B, Ekblom B (1986) Effect of long-term anemia and retransfusion on central circulation during exercise. J Appl Physiol 61:1358-1362. https://doi.org/10.1152/ jappl.1986.61.4.1358

Cerretelli P, di Prampero PE (1987) Gas exchange at exercise. In: Farhi LE, Tenney SM (eds) Handbook of physiology, the respiratory system IV. American Physiological Society, Bethesda, pp 555-632

Cerretelli P, Sikand R, Farhi LE (1966) Readjustments in cardiac output and gas exchange during onset of exercise and recovery. J Appl Physiol 21:1345-1350. https://doi.org/10.1152/jappl.1966. 21.4.1345

Cerretelli P, di Prampero PE, Piiper J (1969) Energy balance of anaerobic work in the dog gastrocnemius muscle. Am J Physiol 217:581-585. https://doi.org/10.1152/ajplegacy.1969.217.2.581

Cerretelli P, Pendergast DR, Paganelli WC, Rennie DW (1979) Effects of specific muscle training on $\dot{V} O_{2}$ on-response and early blood lactate. J Appl Physiol 47:761-768. https://doi.org/10.1152/jappl. 1979.47.4.761

Chang CM, Cassuto Y, LE Pendergast F (1994) Cardiorespiratory response to lower body negative pressure. Aviat Space Environ Med 65:615-620

Chauveau M, Kaufmann M (1887) Expériences pour la determination du coefficient de l'activité nutritive et respiratoire des muscles en repos et en travail. CR Acad Sci 104:1126-1132 
Chauveau M, Tissot J (1896) L'énergie dépensée par le muscle en contraction statique pour le soutien d'une charge d'après les échanges respiratoires. CR Acad Sci 123:1236-1241

Chin LMK, Heigenhauser GJF, Paterson DH, Kowalchuk JM (2010) Pulmonary $\mathrm{O}_{2}$ uptake and leg blood flow kinetics during moderate exercise are slowed by hyperventilation-induced hypocapnic alkalosis. J Appl Physiol 108:1641-1650. https://doi.org/10. 1152/japplphysiol.01346.2009

Chouchou F, Pichot V, Costes F, Guillot M, Barthélémy JC, Bertoletti L, Roche F (2020) Autonomic cardiovascular adaptations to acute head-out water immersion, head-down tilt and supine position. Eur J Appl Physiol 120:337-347. https://doi.org/10. 1007/s00421-019-04278-4

Christmas KM, Bassingthwaighte JB (2017) Equations for $\mathrm{O}_{2}$ and $\mathrm{CO}_{2}$ solubilities in saline and plasma: combining temperature and density dependences. J Appl Physiol 122:1313-1320. https:// doi.org/10.1152/japplphysiol.01124.2016

Chung DC, Niranjan SC, Clark JW Jr, Bidani A, Johnson WE, Zwischenberger JB, Traber DL (1997) A dynamic model of ventricular interaction and pericardial influence. Am J Physiol Heart Circ Physiol 272:H2942-H2962. https://doi.org/10.1152/ ajpheart.1997.272.6.H2942

Clark AR, Tawhai MH, Hoffman EA, Burrowes KS (2011) The interdependent contributions of gravitational and structural features to perfusion distribution in a multiscale model of the pulmonary circulation. J Appl Physiol 110:943-955. https://doi.org/ 10.1152/japplphysiol.00775.2010

Cleuziou C, Perrey S, Lecoq AM, Candau R, Courteix D, Obert P (2005) $\mathrm{O}_{2}$ uptake kinetics during moderate and heavy intensity exercise in humans: the influence of hypoxia and training status. Int J Sport Med 26:356-362. https://doi.org/10. 1055/s-2004-821158

Clifford PS (2007) Skeletal muscle vasodilatation at the onset of exercise. J Physiol 583:825-833. https://doi.org/10.1113/jphys iol.2007.135673

Comroe JH (1944) The hyperpnea of muscular exercise. Physiol Rev 24:319-339. https://doi.org/10.1152/physrev.1944.24.3.319

Comroe JH, Schmidt CF (1943) Reflexes of the limbs as a factor in the hyperpnoea of muscular exercise. Am J Physiol 138:536547. https://doi.org/10.1152/physrev.1944.24.3.319

Constantinopol M, Jones JH, Weibel ER, Taylor CR, Lindholm A, Karas RH (1989) Oxygen transport during exercise in large mammals. II. Oxygen uptake by the pulmonary gas exchanger. J Appl Physiol 67:871-878. https://doi.org/10.1152/jappl.1989. 67.2.871

Convertino VA, Doerr DF, Ludwig DA, Vernikos J (1994) Effect of simulated microgravity on cardiopulmonary baroreflex control of forearm vascular resistance. Am J Physiol Regul Integr Comp Physiol 266:R1962-R1969. https://doi.org/10.1152/ ajpregu.1994.266.6.R1962

Costalat G, Coquart J, Castres I, Tourny C, Lemaître F (2013) Hemodynamic adjustments during breath-holding in trained divers. Eur J Appl Physiol 113:2523-2529. https://doi.org/10.1007/ s00421-013-2690-z

Coste B, Xiao B, Santos JS, Syeda R, Grandl J, Spencer KS, Kim SE, Schmidt M, Mathur J, Dubin AE, Montal M, Patapoutian A (2012) Piezo proteins are pore-forming subunits of mechanically activated channels. Nature 483:176-181. https://doi.org/ 10.1038/nature 10812

Cottin F, Médigue C, Papelier Y (2008) Effect of heavy exercise on spectral baroreflex sensitivity, heart rate, and blood pressure variability in well-trained humans. Am J Physiol Heart Circ Physiol 295:H1150-H1155. https://doi.org/10.1152/ajpheart. 00003.2008
Crandall CG (2000) Carotid baroreflex responsiveness in heat-stressed humans. Am J Physiol Heart Circ Physiol 279:H1955-H1962. https://doi.org/10.1152/ajpheart.2000.279.4.H1955

Crecelius AR, Kirby BS, Richards JC, Garcia LJ, Voyles WF, Larson DG, Luckasen GJ, Dinenno FA (2011a) Mechanisms of ATPmediated vasodilation in humans: modest role for nitric oxide and vasodilating prostaglandins. Am J Physiol Heart Circ Physiol 301:H1302-H1310. https://doi.org/10.1152/ajpheart.00469.2011

Crecelius AR, Kirby BS, Voyles WF, Dinenno FA (2011b) Augmented skeletal muscle hyperaemia during hypoxic exercise in humans is blunted by combined inhibition of nitric oxide and vasodilating prostaglandins. J Physiol 589:3671-3683. https://doi.org/10. 1113/jphysiol.2011.209486

Cummin AR, Iyawe VI, Mehta N, Saunders KB (1986) Ventilation and cardiac output during the onset of exercise, and during voluntary hyperventilation, in humans. J Physiol 370:567-583. https://doi. org/10.1113/jphysiol.1986.sp015951

Cunningham DJ, Petersen ES, Peto R, Pickering TG, Sleight P (1972) Comparison of the effect of different types of exercise on the baroreflex regulation of heart rate. Acta Physiol Scand $86: 444-455$

D’Angelo E, Torelli G (1971) Neural stimuli increasing respiration during different types of exercise. J Appl Physiol 30:116-121. https://doi.org/10.1152/jappl.1971.30.1.116

Danilewski A (1880) Thermodynamische Untersuchungen der Muskeln. Pflüg Arch 21:109-152. https://doi.org/10.1007/BF016 37000

Davies CTM, di Prampero PE, Cerretelli P (1972) Kinetics of cardiac output and respiratory gas exchange during exercise and recovery. J Appl Physiol 32:618-625. https://doi.org/10.1152/ jappl.1972.32.5.618

De Cort SC, Innes JA, Barstow TJ, Guz A (1991) Cardiac output, $\mathrm{O}_{2}$ consumption and arterio-venous $\mathrm{O}_{2}$ difference following a sudden rise in exercise level in humans. J Physiol 441:501-512. https://doi.org/10.1113/jphysiol.1991.sp018764

Degtyarenko AM, Kaufman MP (2006) Barosensory cells in the nucleus tractus solitarius receive convergent input from group III muscle afferents and central command. Neuroscience 140:1041-1050. https://doi.org/10.1016/j.neuroscience.2006. 02.050

Dejours P (1964) Control of respiration in muscular exercise. In: Fenn WO, Rahn H (eds) Handbook of physiology, respiration. American Physiological Society, Washington, pp 631-648

DeLorey DS, Kowalchuk JM, Paterson DH (2003) Relationship between pulmonary $\mathrm{O}_{2}$ uptake kinetics and muscle deoxygenation during moderate-intensity exercise. J Appl Physiol 95:113120. https://doi.org/10.1152/japplphysiol.00956.2002

Delp MD, Laughlin MH (1998) Regulation of skeletal muscle perfusion during exercise. Acta Physiol Scand 162:411-419. https:// doi.org/10.1046/j.1365-201X.1998.0324e.x

Delp MD, O'Leary DS (2004) Integrative control of the skeletal muscle microcirculation in the maintenance of arterial pressure during exercise. J Appl Physiol 97:1112-1118. https://doi.org/10.1152/ japplphysiol.00147.2003

Dempsey JA, Wagner PD (1999) Exercise - induced arterial hypoxemia. J Appl Physiol 87:1997-2006. https://doi.org/10.1152/ jappl.1999.87.6.1997

Dempsey JA, Hanson PG, Henderson KS (1984) Exercise-induced arterial hypoxaemia in healthy human subjects at sea level. J Physiol 355:161-175. https://doi.org/10.1113/jphysiol.1984.sp015412

Di Carlo SE, Bishop VS (1992) Onset of exercise shifts operating point of arterial baroreflex to higher pressures. Am J Physiol Heart Circ Physiol 262:H303-H307. https://doi.org/10.1152/ajpheart. 1992.262.1.H303

Di Rienzo M, Castiglioni P, Iellamo F, Volterrani M, Pagani M, Mancia G, Karemaker JM, Parati G (2008) Dynamic adaptation 
of cardiac baroreflex sensitivity to prolonged exposure to microgravity: data from a 16-day spaceflight. J Appl Physiol 105:1569-1575. https://doi.org/10.1152/japplphysiol.90625. 2008

di Prampero PE (1981) Energetics of muscular exercise. Rev Physiol Biochem Pharmacol 89:143-222. https://doi.org/10.1007/BFb00 35266

di Prampero PE (1986) The energy cost of human locomotion on land and in water. Int J Sports Med 7:55-72. https://doi.org/10.1055/s2008-1025736

di Prampero PE (2000) Cycling on Earth, in Space, on the Moon. Eur J Appl Physiol 82:345-360. https://doi.org/10.1007/s004210000 220

di Prampero PE (2015) La locomozione umana su terra, in acqua, in aria: fatti e teorie. Edi Ermes, Milano

di Prampero PE, Ferretti G (1999) The energetics of anaerobic muscle metabolism: a reappraisal of older and recent concepts. Respir Physiol 118:103-115. https://doi.org/10.1016/s0034-5687(99) 00083-3

di Prampero PE, Margaria R (1968) Relationship between $\mathrm{O}_{2}$ consumption, high energy phosphates and the kinetics of $\mathrm{O}_{2}$ debt in exercise. Pflüg Arch 304:11-19. https://doi.org/10.1007/BF00586714

di Prampero PE, Davies CTM, Cerretelli P, Margaria R (1970) An analysis of $\mathrm{O}_{2}$ debt contracted in submaximal exercise. J Appl Physiol 29:547-551. https://doi.org/10.1152/jappl.1970.29.5. 547

di Prampero PE, Boutellier U, Pietsch P (1983) Oxygen deficit and stores at onset of muscular exercise in humans. J Appl Physiol 55:146-153. https://doi.org/10.1152/jappl.1983.55.1.146

di Prampero PE, Francescato MP, Cettolo V (2003) Energetics of muscular exercise at work onset: the steady state approach. Pflüg Arch 445:741-746. https://doi.org/10.1007/s00424-002-0991-x

Dominelli PB, Foster GE, Dominelli GS, Henderson WR, Koehle MS, McKenzie DC, Sheel AW (2013) Exercise-induced arterial hypoxaemia and the mechanics of breathing in healthy young women. J Physiol 591:3017-3034. https://doi.org/10.1113/jphys iol.2013.252767

Domino KB, Eisenstein BL, Cheney FW, Hlastala MP (1991) Pulmonary blood flow and ventilation-perfusion heterogeneity. J Appl Physiol 71:252-258. https://doi.org/10.1152/jappl.1991.71.1.252

Domino KB, Eisenstein BL, Tran T, Hlastala MP (1993) Increased pulmonary perfusion worsens ventilation-perfusion matching. Anesthesiology 79:817-826. https://doi.org/10.1097/00000542199310000-00025

Dora KA (2016) Endothelial-smooth muscle cell interactions in the regulation of vascular tone in skeletal muscle. Microcirculation 23:626-630. https://doi.org/10.1111/micc.12322

Eckberg DL, Sleight P (1992) Human baroreflex in health and disease. Clarendon Press, Oxford

Eggleton P, Eggleton GP (1927a) The inorganic phosphate and a labile form of inorganic phosphate in the gastrocnemius muscle of the dog. Biochem J 21:190-195

Eggleton P, Eggleton GP (1927b) The physiological significance of phosphate. J Physiol 63:155-161. https://doi.org/10.1113/jphys iol.1927.sp002391

Eiken O, Bjurstedt H (1985) Cardiac responses to lower body negative pressure and dynamic leg exercise. Eur J Appl Physiol 54:451455. https://doi.org/10.1007/BF00422950

Ekblom B, Hermansen L (1968) Cardiac output in athletes. J Appl Physiol 25:619-625. https://doi.org/10.1152/jappl.1968.25.5.619

Ekblom B, Åstrand PO, Saltin B, Stenberg J, Wallström B (1968) Effect of training on circulatory response to exercise. J Appl Physiol 24:518-528. https://doi.org/10.1152/jappl.1968.24.4.518

Ekblom B, Goldbarg AN, Kilbom A, Åstrand PO (1972) Effects of atropine and propranolol on the oxygen transport system during exercise in man. Scand J Clin Lab Invest 30:35-42. https://doi. org/10.3109/00365517209081087

Ekblom B, Huot R, Stein EM, Thorstensson AT (1975) Effect of changes in arterial oxygen content on circulation and physical performance. J Appl Physiol 39:71-75. https://doi.org/10.1152/ jappl.1975.39.1.71

Ekblom B, Wilson G, Åstrand PO (1976) Central circulation during exercise after venesection and reinfusion of red blood cells. J Appl Physiol 40:379-383. https://doi.org/10.1152/jappl.1976. 40.3.379

Eldridge FL, Millhorn DE, Waldrop TG (1981) Exercise hyperpnea and locomotion: parallel activation from the hypothalamus. Science 211:844-846. https://doi.org/10.1126/science.7466362

Elia A, Wilson OJ, Lees M, Parker PJ, Barlow MJ, Cocks M, O’Hara JP (2019) Skeletal muscle, haematological and splenic volume characteristics of elite breath-hold divers. Eur J Appl Physiol 119:2499-2511. https://doi.org/10.1007/s00421-019-04230-6

Elia A, Barlow MJ, Wilson OJ, O'Hara JP (2021a) Six weeks of dynamic apnoeic training stimulates erythropoiesis but does not increase splenic volume. Eur J Appl Physiol 121:827-838. https://doi.org/10.1007/s00421-020-04565-5

Elia A, Barlow MJ, Wilson OJ, O'Hara JP (2021b) Splenic responses to a series of repeated maximal static and dynamic apnoeas with whole-body immersion in water. Exp Physiol 106:338-349. https://doi.org/10.1113/EP088404

Elia A, Gennser M, Harlow PS, Lees MJ (2021c) Physiology, pathophysiology and (mal)adaptations to chronic apnoeic training: a state-of-the-art review. Eur J Appl Physiol 121:1543-1566. https://doi.org/10.1007/s00421-021-04664-x

Ellis CG, Wrigley SM, Groom AC (1994) Heterogeneity of red blood cell perfusion in capillary networks supplied by a single arteriole in resting skeletal muscle. Circ Res 75:357-368. https://doi.org/ 10.1161/01.res.75.2.357

Ellsworth ML, Forrester T, Ellis CG, Dietrich HH (1995) The erythrocyte as a regulator of vascular tone. Am J Physiol Heart Circ Physiol 269:H2155-H2161. https://doi.org/10.1152/ajpheart. 1995.269.6.H2155

Elstad M, Nådland IH, Toska K, Walløe L (2009) Stroke volume decreases during mild dynamic and static exercise in supine humans. Acta Physiol 195:289-300. https://doi.org/10.1111/j. 1748-1716.2008.01887.x

Embden G, Lawaczeck H (1922) Über die Bildung anorganischer Phosphorsäure bei del Kontraktion des Froschmuskels. Biochem Z 127:181-199

Engelen M, Porszasz J, Riley M, Wasserman K, Maehara K, Barstow TJ (1996) Effects of hypoxic hypoxia on $\mathrm{O}_{2}$ uptake and heart rate kinetics during heavy exercise. J Appl Physiol 81:2500-2508. https://doi.org/10.1152/jappl.1996.81.6.2500

Engelhardt VA, Lyubimova MN (1939) Myosin and adenosine-triphosphatase. Nature 144:668-669

Engelmann TW (1878) Neue Untersuchungen über die mikroskopischen Vorgänge bei der Muskelcontraktion. Pflüg Arch 18:1-25. https://doi.org/10.1007/BF01637167

Engelmann TW (1895) Croonian Lecture. On the nature of muscular contraction. Proc R Soc Lond B 57:411-435. https://doi.org/10. 1098/rspl.1894.0171

Eriksen M, Waaler BA, Walloe L, Wesche J (1990) Dynamics and dimensions of cardiac output changes in humans at the onset and the end of moderate rhythmic exercise. J Physiol 426:423-437. https://doi.org/10.1113/jphysiol.1990.sp018147

Fadel PJ, Raven PB (2012) Human investigations into the arterial and cardiopulmonary baroreflexes during exercise. Exp Physiol 97:39-50. https://doi.org/10.1113/expphysiol.2011.057554

Fagoni N, Sivieri A, Antonutto G, Moia C, Taboni A, Bringard A, Ferretti G (2015) Cardiovascular responses to dry resting apnoeas in 
elite divers while breathing pure oxygen. Respir Physiol Neurobiol 219:1-8. https://doi.org/10.1016/j.resp.2015.07.016

Fagoni N, Taboni A, Vinetti G, Bottarelli S, Moia C, Bringard A, Ferretti G (2017) Alveolar gas composition during maximal and interrupted apnoeas in ambient air and pure oxygen. Respir Physiol Neurobiol 235:45-51. https://doi.org/10.1016/j.resp. 2016.10.002

Fagoni N, Bruseghini P, Adami A, Capelli C, Lador F, Moia C, Tam E, Bringard A, Ferretti G (2020) Effect of lower body negative pressure on phase I cardiovascular responses at exercise onset. Int J Sports Med 41:209-218. https://doi.org/10.1055/a-1028-7496

Fagraeus L, Linnarsson D (1976) Autonomic origin of heart rate fluctuations at the onset of muscular exercise. J Appl Physiol 40:679-682. https://doi.org/10.1152/jappl.1976.40.5.679

Faisal A, Beavers KR, Robertson AD, Hughson RL (2009) Prior moderate and heavy exercise accelerate oxygen uptake and cardiac output kinetics in endurance athletes. J Appl Physiol 106:15531563. https://doi.org/10.1152/japplphysiol.91550.2008

Faisal A, Beavers KR, Hughson RL (2010) $\mathrm{O}_{2}$ uptake and blood pressure regulation at the onset of exercise: interaction of circadian rhythm and priming exercise. Am J Physiol Heart Circ Physiol 299:H1832-H1842. https://doi.org/10.1152/ajpheart.00762.2010

Farhi LE, Rahn H (1955) A theoretical analysis of the alveolar-arterial $\mathrm{O}_{2}$ difference, with special reference to the distribution effect. J Appl Physiol 7:699-703. https://doi.org/10.1152/jappl. 1955.7.6.699

Farhi LE, Rahn H (1960) Dynamics of changes in carbon dioxide stores. Anesthesiology 21:604-614. https://doi.org/10.1097/ 00000542-196011000-00004

Favier R, Desplanches D, Frutoso J, Grandmontagne M, Flandrois $\mathrm{R}$ (1983) Ventilatory transients during exercise: peripheral or central control? Pflüg Arch 396:269-276. https://doi.org/10. 1007/BF01063930

Fenn WO, Rahn H, Otis AB (1946) A theoretical study of the composition of the alveolar air at altitude. Am J Physiol 146:637-653. https://doi.org/10.1152/ajplegacy.1946.146.5.637

Ferguson BS, Rogatzki MJ, Goodwin ML, Kane DA, Rightmire Z, Gladden LB (2018) Lactate metabolism: historical context, prior misinterpretations, and current understanding. Eur J Appl Physiol 118:691-728. https://doi.org/10.1007/ s00421-017-3795-6

Fernandez-Verdejo R, Bajpeyi S, Ravussin E, Galgani JE (2018) Metabolic flexibility to lipid availability during exercise is enhanced in individuals with high insulin sensitivity. Am J Physiol Endocrinol Metab 315:E715-E722. https://doi.org/10.1152/ajpendo. 00126.2018

Ferretti G (2001) Extreme human breath-hold diving. Eur J Appl Physiol 84:254-271. https://doi.org/10.1007/s004210000377

Ferretti G (2014) Maximal oxygen consumption in healthy humans: theories and facts. Eur J Appl Physiol 114:2007-2036. https:// doi.org/10.1007/s00421-014-2911-0

Ferretti G (2015) Energetics of muscular exercise. Springer, Heidelberg

Ferretti G, Gussoni M, di Prampero PE, Cerretelli P (1987) Effects of exercise on maximal instantaneous muscular power of humans. J Appl Physiol 62:2288-2294. https://doi.org/10.1152/jappl.1987. 62.6.2288

Ferretti G, Boutellier U, Pendergast DR, Moia C, Minetti AE, Howald H, di Prampero PE (1990) Muscular exercise at high altitude. IV. Oxygen transport system before and after exposure to chronic hypoxia. Int J Sports Med 11:S15-S20. https://doi.org/10.1055/s2007-1024848

Ferretti G, Costa M, Ferrigno M, Grassi B, Marconi C, Lundgren CE, Cerretelli P (1991) Alveolar gas composition and exchange during deep breath-hold diving and dry breath holds in elite divers. J Appl Physiol 70:794-802. https://doi.org/10.1152/jappl.1991. 70.2 .794
Ferretti G, Kayser B, Schena F, Turner DL, Hoppeler H (1992) Regulation of perfusive $\mathrm{O}_{2}$ transport during exercise in humans: effects of changes in haemoglobin concentration. J Physiol 455:679688. https://doi.org/10.1113/jphysiol.1992.sp019322

Ferretti G, Binzoni T, Hulo N, Kayser B, Thomet JM, Cerretelli P (1995) Kinetics of oxygen consumption during maximal exercise at different muscle temperatures. Respir Physiol 102:261-268. https://doi.org/10.1016/0034-5687(95)00071-2

Ferretti G, Licker MJ, Anchisi S, Moia C, Susta D, Morel DR (2005) The effects of beta1-adrenergic blockade on cardiovascular oxygen flow in normoxic and hypoxic humans at exercise. Eur J Appl Physiol 95:250-259. https://doi.org/10.1007/s00421-005-1393-5

Ferretti G, Fagoni N, Taboni A, Bruseghini P, Vinetti G (2017) The physiology of submaximal exercise: the steady state concept. Respir Physiol Neurobiol 246:76-85. https://doi.org/10.1016/j. resp.2017.08.005

Fick A (1870) Über die Messung des Blutquantums in den Herzventrikeln. Physikalisch Medizin Gesellschaft, Würzburg

Fick A (1893) Einige Bemerkungen zu Engelmann's Abhandlung über den Ursprung der Muskelkraft. Pflüg Arch 53:606-615. https:// doi.org/10.1007/BF01795654

Fisher JP, Young CN, Fadel PJ (2015) Autonomic adjustments to exercise in humans. Compr Physiol 5:475-512. https://doi.org/10. 1002/cphy.c140022

Fiske CH, Subbarow Y (1927) The nature of inorganic phosphate in the voluntary muscle. Science 65:401-403. https://doi.org/10. 1126/science.65.1686.401

Fiske CH, Subbarow Y (1928) The isolation and function of phosphocreatine. Science 67:169-171. https://doi.org/10.1126/science. 67.1728 .169

Fitz-Clarke JR (2007) Mechanics of airway and alveolar collapse in human breath-hold diving. Respir Physiol Neurobiol 159:202210. https://doi.org/10.1016/j.resp.2007.07.006

Fitz-Clarke JR (2009) Lung compression effects on gas exchange in human breath-hold diving. Respir Physiol Neurobiol 165:221228. https://doi.org/10.1016/j.resp.2008.12.006

Fitz-Clarke JR (2018) Breath-hold diving. Compr Physiol 8:585-630. https://doi.org/10.1002/cphy.c160008

Fletcher WM, Hopkins FG (1907) Lactic acid in amphibian muscle. J Physiol 35:247-309. https://doi.org/10.1113/jphysiol.1907. sp001194

Fletcher WM, Hopkins FG (1917) Croonian Lecture of 1915: the respiratory processes in muscle and the nature of muscular motion. Proc R Soc Lond B 89:444-467. https://doi.org/10.1098/rspb. 1917.0005

Fontolliet T, Pichot V, Bringard A, Fagoni N, Adami A, Tam E, Furlan R, Barthélémy JC, Ferretti G (2018) Testing the vagal withdrawal hypothesis during light exercise under autonomic blockade: a heart rate variability study. J Appl Physiol 125:1804-1811. https://doi.org/10.1152/japplphysiol.00619.2018

Fontolliet T, Bringard A, Adami A, Fagoni N, Tam E, Taboni A, Ferretti G (2021) Vagal blockade suppresses the phase I heart rate response but not the phase I cardiac output response at exercise onset in humans. Eur J Appl Physiol 121:3173-3187. https://doi. org/10.1007/s00421-021-04769-3

Francescato MP, Cettolo V, di Prampero PE (2008) Influence of phosphagen concentration on phosphocreatine breakdown kinetics. Data from human gastrocnemius muscle. J Appl Physiol 105:158-164. https://doi.org/10.1152/japplphysiol.00007.2008

Francescato MP, Cettolo V, di Prampero PE (2013) O 2 uptake kinetics at work onset: role of cardiac output and of phosphocreatine breakdown. Respir Physiol Neurobiol 185:287-295. https://doi. org/10.1016/j.resp.2012.09.015

Gale GE, Torre-Bueno JR, Moon RE, Saltzman HA, Wagner PD (1985) Ventilation-perfusion inequality in normal humans 
during exercise at sea level and simulated altitude. J Appl Physiol 58:978-988. https://doi.org/10.1152/jappl.1985.58.3.978

Galgani JE, Moro C, Ravussin E (2008) Metabolic flexibility and insulin resistance. Am J Physiol Endocrinol Metab 295:E1009E1017. https://doi.org/10.1152/ajpendo.90558.2008

Galgani JE, Johanssen NM, Bajpeyi S, Costford SR, Zhang Z, Gupta AK, Ravussin E (2012) Role of skeletal muscle mitochondrial density on exercise-stimulated lipid oxidation. Obesity 20:13871393. https://doi.org/10.1038/oby.2011.166

Gallagher KM, Fadel PJ, Smith SA, Strømstad M, Ide K, Secher NH, Raven PB (2006) The interaction of central command and the exercise pressor reflex in mediating baroreflex resetting during exercise in humans. Exp Physiol 91:79-87. https://doi.org/10. 1113/expphysiol.2005.032110

Garry RC, Sloan AW, De Weir JB, Wishart M (1954) The concentration of haemoglobin in the blood of young adult men and women: the effect of administering small doses of iron for prolonged periods. Br J Nutr 8:253-268. https://doi.org/10.1079/bjn19540038

Geppert J, Zuntz N (1888) Über die Regulation der Atmung. Pflüg Arch 42:189-244. https://doi.org/10.1007/BF01669357

Ghisla S (2004) Beta-oxidation of fatty acids. Eur J Biochem 271:459 461. https://doi.org/10.1046/j.1432-1033.2003.03952.x

Gilbert R, Auchincloss JH Jr, Baule GH (1967) Metabolic and circulatory adjustments to unsteady-state exercise. J Appl Physiol 22:905-912. https://doi.org/10.1152/jappl.1967.22.5.905

Gisolfi C, Robinson S, Turrell ES (1966) Effects of aerobic work performed during recovery from exhausting work. J Appl Physiol 21:1767-1772. https://doi.org/10.1152/jappl.1966.21.6.1767

Gledhill N, Froese AB, Buick FJ, Bryan AC (1978) $\dot{V}_{A} / \dot{Q}$ inhomogeneity and $\mathrm{aaDO}_{2}$ in man during exercise: effect of $\mathrm{SF}_{6}$ breathing. J Appl Physiol 45:512-515. https://doi.org/10.1152/jappl.1978. 45.4.512

Glenny RW, Robertson HT (1990) Fractal properties of pulmonary blood flow: characterization of spatial heterogeneity. J Appl Physiol 69:532-545. https://doi.org/10.1152/jappl.1990.69.2.532

Glenny RW, Robertson HT (2011) Spatial distribution of ventilation and perfusion: mechanisms and regulation. Compr Physiol 1:375-395. https://doi.org/10.1002/cphy.c100002

Gonzalez-Alonso J, Richardson RS, Saltin B (2001) Exercising skeletal muscle blood flow in humans responds to reduction in arterial oxyhaemoglobin, but not to altered free oxygen. J Physiol 530:331-341. https://doi.org/10.1111/j.1469-7793.2001.03311.x

Gonzalez-Alonso J, Olsen DB, Saltin B (2002) Erythrocyte and the regulation of human skeletal muscle blood flow and oxygen delivery: role of circulating ATP. Circ Res 91:1046-1055. https:// doi.org/10.1161/01.res.0000044939.73286.e2

Goulding RP, Okushima D, Fukuoka Y, Marwood S, Kondo N, Poole DC, Barstow TJ, Koga S (2021) Impact of supine versus upright exercise on muscle deoxygenation heterogeneity during ramp incremental cycling is site specific. Eur J Appl Physiol 121:1283-1296. https://doi.org/10.1007/s00421-021-04607-6

Grassi B (2000) Skeletal muscle on-kinetics: set by $\mathrm{O}_{2}$ delivery or by $\mathrm{O}_{2}$ utilization? New insights into an old issue. Med Sci Sports Exerc 32:108-116. https://doi.org/10.1097/00005768-20000 $1000-00017$

Grassi B (2003) Oxygen uptake kinetics: old and recent lessons from experiments on isolated muscle in situ. Eur J Appl Physiol 90:242-249. https://doi.org/10.1007/s00421-003-0994-0

Grassi B, Poole DC, Richardson RS, Knight DR, Erickson BK, Wagner PD (1996) Muscle $\mathrm{O}_{2}$ uptake kinetics in humans: implications for metabolic control. J Appl Physiol 80:988-998. https://doi.org/10. 1152/jappl.1996.80.3.988

Grassi B, Gladden LB, Samaja M, Stary CM, Hogan MC (1998) Faster adjustment of $\mathrm{O}_{2}$ delivery does not affect -on kinetics in isolated in situ canine muscle. J Appl Physiol 85:1394-1403. https://doi. org/10.1152/jappl.1998.85.4.1394
Grassi B, Hogan MC, Greenhaff PL, Hamann JJ, Kelley KM, Aschenbach WG, Constantin-Teodosiu D, Gladden LB (2002) $\dot{V} \mathrm{O}_{2}$ on-kinetics in dog gastrocnemius in situ following activation of pyruvate dehydrogenase by dichloroacetate. J Physiol 538:195207. https://doi.org/10.1113/jphysiol.2001.012984

Grassi B, Hogan MC, Kelley KM, Howlett RA, Gladden LB (2005) Effect of nitric oxide synthase inhibition by L-NAME on oxygen uptake kinetics in isolated canine muscle in situ. J Physiol 568:1021-1033. https://doi.org/10.1113/jphysiol.2005.090068

Grassi B, Rossiter HR, Hogan MC, Howlett RA, Harris JE, Goodwin ML, Dobson JL, Gladden LB (2011) Faster $\mathrm{O}_{2}$ uptake kinetics in canine skeletal muscle in situ after acute creatine kinase inhibition. J Physiol 589:221-233. https://doi.org/10.1113/jphys iol.2010.195164

Greenhaff PL (2001) The creatine-phosphocreatine system: there's more than one song in its repertoire. J Physiol 537:657. https:// doi.org/10.1111/j.1469-7793.2001.00657.x

Grønlund J (1984) A new method for breath-to-breath determination of oxygen flux across the alveolar membrane. Eur J Appl Physiol 52:167-172. https://doi.org/10.1007/BF00433387

Guyenet PG (2014) Regulation of breathing and autonomic outflows by chemoreceptors. Compr Physiol 4:1511-1562. https://doi.org/ 10.1002/cphy.c140004

Hakim TS, Gilbert E, Camporesi EM (1994) Blood flow, volume, and transit time in the pulmonary microvasculature using laserDoppler. J Appl Physiol 76:2643-2650. https://doi.org/10.1152/ jappl.1994.76.6.2643

Hammond MD, Gale GE, Kapitan KS, Ries A, Wagner PD (1986) Pulmonary gas exchange in humans during exercise at sea level. J Appl Physiol 60:1590-1598. https://doi.org/10.1152/ jappl.1986.60.5.1590

Hanukoglu I (2017) ASIC and ENaC type sodium channels: conformational states and the structures of the ion selectivity filter. FEBS J 284:525-545. https://doi.org/10.1111/febs.13840

Harf A, Pratt T, Hughes JMB (1978) Regional distribution of $\dot{V}_{A} / \dot{Q}$ in man at rest and with exercise measured with krypton- $81 \mathrm{~m}$. J Appl Physiol 44:115-123. https://doi.org/10.1152/jappl.1978. 44.1.115

Harms CA, McClaran SR, Nickele GA, Pegelow DF, Nelson WB, Dempsey JA (1998) Exercise-induced arterial hypoxaemia in healthy young women. J Physiol 507:619-628. https://doi.org/ 10.1111/j.1469-7793.1998.619bt.x

Harrop GA (1919) The oxygen and carbon dioxide content of arterial and of venous blood in normal individuals and in patients with anemia and heart disease. J Exp Med 30:241-257. https://doi. org/10.1084/jem.30.3.241

Hartley LH, Vogel JA, Landowne L (1973) Central, femoral and brachial circulation during exercise in hypoxia. J Appl Physiol 34:87-90. https://doi.org/10.1152/jappl.1973.34.1.87

Hearon CM Jr, Dinenno FA (2016) Regulation of skeletal muscle blood flow during exercise in ageing humans. J Physiol 594:2261-2273. https://doi.org/10.1113/JP270593

Heidenhain R (1864) Mechanische Leistung, Wärmeentwiclung und Stoffumsatz bei der Muskeltätigkeit. Breitkopf u. Härtel, Leipzig

Heinemann HN (1901) Experimentelle Untersuchungen am Menschen über den Einfluss der Muskelarbeit auf den Stoffverbrauch und die Bedeutung der einzelnen Nährstoffe als Quelle der Muskelkraft. Pflüg Arch 83:441-476. https://doi.org/10.1007/BF017 46505

Heinonen I, Nesterov SV, Kemppainen J, Nuutila P, Knuuti J, Laitio R, Kjaer M, Boushel R, Kalliokoski KK (2007) Role of adenosine in regulating the heterogeneity of skeletal muscle blood flow during exercise in humans. J Appl Physiol 103:2042-2048. https://doi. org/10.1152/japplphysiol.00567.2007 
Heinonen I, Duncker DJ, Knuuti J, Kalliokoski KK (2012) The effect of acute exercise with increasing workloads on inactive muscle blood flow and its heterogeneity in humans. Eur J Appl Physiol 112:3503-3509. https://doi.org/10.1007/s00421-012-2329-5

Heinonen I, Savolainen AM, Han C, Kemppainen J, Oikonen V, Luotolahti M, Duncker DJ, Merkus D, Knuuti J, Kalliokoski KK (2013) Pulmonary blood flow and its distribution in highly trained endurance athletes and healthy control subjects. J Appl Physiol 114:329-334. https://doi.org/10.1152/japplphysiol. 00710.2012

Heller H, Schuster HD (2007) Model analysis on alveolar-capillary $\mathrm{O}_{2}$ equilibration during exercise. Nitric Oxide 16:131-134. https:// doi.org/10.1016/j.niox.2006.06.006

Helmoltz H (1847) Über die Erhaltung der Kraft. G. Reimer, Berlin

Hermansen L, Ekblom B, Saltin B (1970) Cardiac output during submaximal and maximal treadmill and bicycle exercise. J Appl Physiol 29:82-86. https://doi.org/10.1152/jappl.1970.29.1.82

Hill AV (1910) The possible effects of the aggregation of the molecules of haemoglobin on its dissociation curves. J Physiol 40:iv-vii

Hill AV (1913) The energy degraded in the recovery processes of stimulated muscles. J Physiol 46:28-80. https://doi.org/10.1113/jphys iol.1913.sp001578

Hill AV (1916) Die Beziehungen zwischen der Wärmebildung und dem im Muskel stattfindenden chemischen Prozessen. Ergeb Physiol 15:340-479

Hill AV (1922) The maximum work and mechanical efficiency of human muscles and their most economical speed. J Physiol 56:19-41. https://doi.org/10.1113/jphysiol.1922.sp001989

Hill AV (1932) The revolution in muscle physiology. Physiol Rev 12:56-67. https://doi.org/10.1152/physrev.1932.12.1.56

Hill AV, Long CNH, Lupton H (1924) Muscular exercise, lactic acid and the supply and utilization of oxygen. Parts IV - VI. Proc R Soc Lond B 97:84-138. https://doi.org/10.1098/rspb.1924. 0045

Hirayanagi K, Iwase S, Kamiya A, Sasaki T, Mano T, Yajima K (2004) Functional changes in autonomic nervous system and baroreceptor reflex induced by 14 days of 6 degrees head-down bed rest. Eur J Appl Physiol 92:160-167. https://doi.org/10. 1007/s00421-004-1067-8

Hlastala MP, Bernard SL, Erickson HH, Fedde MR, Gaughan EM, McMurphy R, Emery MJ, Polissar N, Glenny RW (1996) Pulmonary blood flow distribution in standing horses is not dominated by gravity. J Appl Physiol 81:1051-1061. https://doi.org/ 10.1152/jappl.1996.81.3.1051

Honig CR, Gayeski TE, Connett RJ (1984) Balance between $\mathrm{O}_{2}$ availability and $\dot{V} \mathrm{O}_{2}$ in rest-work transition as measured by myoglobin saturation in subcellular volumes. Adv Exp Med Biol 180:651-661. https://doi.org/10.1007/978-1-4684-4895563

Houston CS, Sutton JR, Cymerman A, Reeves JT (1987) Operation Everest II: man at extreme altitude. J Appl Physiol 63:877-882. https://doi.org/10.1152/jappl.1987.63.2.877

Hughes RL, Clode M, Edwards RH, Goodwin TJ, Jones NL (1968) Effect of inspired $\mathrm{O}_{2}$ on cardiopulmonary and metabolic responses to exercise in man. J Appl Physiol 24:336-347. https:// doi.org/10.1152/jappl.1968.24.3.336

Hughson RL, Kowalchuk JM (1995) Kinetics of $\mathrm{O}_{2}$ uptake for submaximal exercise in hyperoxia, normoxia, and hypoxia. Can $\mathrm{J}$ Appl Physiol 20:198-210. https://doi.org/10.1139/h95-014

Huxley AF (1957) Muscle structure and theories of contraction. Prog Biophys Biophys Chem 7:255-318

Huxley AF (1974) Muscular contraction. J Physiol 243:1-43. https:// doi.org/10.1113/jphysiol.1974.sp010740

Iellamo F, Hughson RL, Castrucci F, Legramante JM, Raimondi G, Peruzzi G, Tallarida G (1994) Evaluation of spontaneous baroreflex modulation of sinus node during isometric exercise in healthy humans. Am J Physiol Heart Circ Physiol 267:H994H1001. https://doi.org/10.1152/ajpheart.1994.267.3.H994

Iellamo F, Legramante JM, Raimondi G, Peruzzi G (1997) Baroreflex control of sinus node during dynamic exercise in humans: effects of central command and muscle reflexes. Am J Physiol Heart Circ Physiol 272:H1157-H1164. https://doi.org/10.1152/ajphe art.1997.272.3.H1157

Iellamo F, Legramante JM, Pigozzi F, Spataro A, Norbiato G, Lucini D, Pagani M (2002) Conversion from vagal to sympathetic predominance with strenuous training in high-performance world class athletes. Circulation 105:2719-2724. https://doi.org/10.1161/01. cir.0000018124.01299.ae

Johansson JE (1895) Uber die Einwirkung der Muskelthätigkeit auf die Athmung und die herzthätigkeit. Skand Arch Physiol 5:20-66. https://doi.org/10.1111/j.1748-1716.1894.tb00192.x

Jones AM, Poole DC (eds) (2005) Oxygen uptake kinetics in sport, exercise and medicine. Routledge, London

Jones AM, Wilkerson DP, DiMenna F, Fulford J, Poole DC (2008) Muscle metabolic responses to exercise above and below the "critical power" assessed using ${ }^{31} \mathrm{P}-\mathrm{MRS}$. Am J Physiol Regul Integr Comp Physiol 294:R585-R593. https://doi.org/10.1152/ ajpregu.00731.2007

Jones AM, Vanhatalo A, Burnley M, Morton RH, Poole DC (2010) Critical power: implications for the determination of $\dot{V} \mathrm{O}_{2} \max$ and exercise tolerance. Med Sci Sports Exerc 42:1876-1890. https://doi.org/10.1249/MSS.0b013e3181d9cf7f

Jones AM, Grassi B, Christensen PM, Krustrup P, Bangsbo J, Poole DC (2011) Slow component of $\dot{V} O_{2}$ kinetics: mechanistic bases and practical applications. Med Sci Sports Exerc 43:2046-2062. https://doi.org/10.1249/MSS.0b013e31821fcfc1

Kawashiro T, Campos Carles A, Perry SF, Piiper J (1975) Diffusivity of various inert gases in rat skeletal muscle. Pflüg Arch 359:219-230. https://doi.org/10.1007/BF00587381

Kelman GR (1966) Digital computer subroutine for the conversion of oxygen tension into saturation. J Appl Physiol 21:1375-1376. https://doi.org/10.1152/jappl.1966.21.4.1375

Kent BB, Drane JW, Blumenstein B, Manning JW (1972) A mathematical model to assess changes in the baroreceptor reflex. Cardiology 57:295-310. https://doi.org/10.1159/000169528

Kölliker A (1888) Zur Kenntnis der quergestreiften Muskelfasern. Z Wissensch Zool 47:689-710

Kornberg H (2000) Krebs and his trinity of cycles. Nat Rev Mol Cell Biol 1:225-228. https://doi.org/10.1038/35043073

Koskolou MD, Calbet JA, Rådegran G, Roach RC (1997a) Hypoxia and cardiovascular response to dynamic knee extensor exercise. Am J Physiol Heart Circ Physiol 273:H1787-H1793. https://doi.org/10.1152/ajpheart.1997.272.6.H2655

Koskolou MD, Roach RC, Calbet JA, Radegran G, Saltin B (1997b) Cardiovascular responses to dynamic exercise with acute anemia in humans. Am J Physiol Heart Circ Physiol 273:H1787H1793. https://doi.org/10.1152/ajpheart.1997.273.4.H1787

Krebs HA (1970) The history of the tricarboxylic acid cycle. Perspect Biol Med 14:154-170. https://doi.org/10.1353/pbm.1970. 0001

Krebs HA, Kornberg H (1957) Energy transformation in living matter. Springer, Berlin

Krogh A (1919) The number and distribution of capillaries in muscles with calculations of the oxygen pressure head necessary for supplying the tissue. J Physiol 52:409-415. https://doi.org/10.1113/ jphysiol.1919.sp001839

Krogh A (1922) The anatomy and physiology of capillaries. Yale University Press, New Haven

Krogh A, Lindhard J (1913a) The volume of the "dead space" in breathing. J Physiol 47:30-43. https://doi.org/10.1113/jphysiol. 1913.sp001610 
Krogh A, Lindhard J (1913b) The regulation of respiration and circulation during the initial stages of muscular work. J Physiol 47:112-136. https://doi.org/10.1113/jphysiol.1913.sp001616

Krogh A, Lindhard J (1917) The volume of the dead space in breathing and the mixing of gases in the lungs of man. J Physiol 51:59-90. https://doi.org/10.1113/jphysiol.1917.sp001785

Kuby SA, Noda L, Lardy HA (1954) Adenosinetriphosphate-creatine transphosphorylase. III. Kinetic studies. J Biol Chem 210:65-82

Lacour JR, Bourdin M (2015) Factors affecting the energy cost of level running at submaximal speed. Eur J Appl Physiol 115:651-673. https://doi.org/10.1007/s00421-015-3115-y

Lador F, Azabji Kenfack M, Moia C, Cautero M, Morel DR, Capelli C, Ferretti G (2006) Simultaneous determination of the kinetics of cardiac output, systemic $\mathrm{O}_{2}$ delivery, and lung $\mathrm{O}_{2}$ uptake at exercise onset in men. Am J Physiol Regul Integr Comp Physiol 290:R1071-R1079. https://doi.org/10.1152/ajpregu.00366.2005

Lador F, Tam E, Azabji-Kenfack M, Cautero M, Moia C, Morel DR, Capelli C, Ferretti G (2008) Phase I dynamics of cardiac output, systemic $\mathrm{O}_{2}$ delivery and lung $\mathrm{O}_{2}$ uptake at exercise onset in men in acute normobaric hypoxia. Am J Physiol Regul Integr Comp Physiol 295:R624-R632. https://doi.org/10.1152/ajpregu. 00797.2007

Lador F, Tam E, Adami A, Azabji-Kenfack M, Bringard A, Cautero M, Moia C, Morel DR, Capelli C, Ferretti G (2013) Cardiac output, $\mathrm{O}_{2}$ delivery and $\mathrm{O}_{2}$ kinetics during step exercise in acute normobaric hypoxia. Respir Physiol Neurobiol 186:206-213. https:// doi.org/10.1016/j.resp.2013.01.017

Lahiri S, Rozanov C, Roy A, Storey B, Buerk DG (2001) Regulation of oxygen sensing in peripheral arterial chemoreceptors. Int $\mathrm{J}$ Biochem Cell Biol 33:755-774. https://doi.org/10.1016/s13572725(01)00042-5

Lanphier EH, Rahn H (1963) Alveolar gas exchange during breathholding with air. J Appl Physiol 18:478-482. https://doi.org/ 10.1152/jappl.1963.18.3.478

Laughlin MH (1987) Skeletal muscle blood flow capacity: Role of muscle pump in exercise hyperemia. Am J Physiol Heart Circ Physiol 253:H993-H1004. https://doi.org/10.1152/ajpheart. 1987.253.5.H993

Leyk D, Essfeld D, Hoffmann U, Wunderlich HG, Baum K, Stegemann J (1994) Postural effect on cardiac output, oxygen uptake and lactate during cycle exercise of varying intensity. Eur $\mathbf{J}$ Appl Physiol 68:30-35. https://doi.org/10.1007/BF00599238

Lin YC, Lally DA, Moore TO, Hong SK (1974) Physiological and conventional breath-hold breaking points. J Appl Physiol 37:291-296. https://doi.org/10.1152/jappl.1974.37.3.291

Lindholm P, Linnarsson D (2002) Pulmonary gas exchange during apnoea in exercising men. Eur J Appl Physiol 86:487-491. https://doi.org/10.1007/s00421-002-0581-9

Lindholm P, Lundgren CEG (2006) Alveolar gas composition before and after maximal breath-holds in competitive divers. Undersea Hyperb Med 33:463-467

Lindholm P, Lundgren CEG (2009) The physiology and pathophysiology of human breath-hold diving. J Appl Physiol 106:284292. https://doi.org/10.1152/japplphysiol.90991.2008

Lindholm P, Sundblad P, Linnarsson D (1999) Oxygen-conserving effects of apnea in exercising men. J Appl Physiol 87:21222127. https://doi.org/10.1152/jappl.1999.87.6.2122

Lindholm P, Nordh J, Linnarsson D (2002) Role of hypoxemia for the cardiovascular responses to apnea during exercise. Am J Physiol Regul Integr Comp Physiol 283:R1227-R1235. https:// doi.org/10.1152/ajpregu.00036.2002

Linér MH, Linnarsson D (1994) Tissue oxygen and carbon dioxide stores and breath-hold diving in humans. J Appl Physiol 77:542-547. https://doi.org/10.1152/jappl.1994.77.2.542
Linnarsson D (1974) Dynamics of pulmonary gas exchange and heart rate changes at start and end of exercise. Acta Physiol Scand Suppl 415:1-78

Linnarsson D, Spaak J, Sundblad P (2006) Baroreflex impairment during rapid posture changes at rest and exercise after 120 days of bed rest. Eur J Appl Physiol 96:37-45. https://doi.org/10. 1007/s00421-005-0062-z

Lohmann K (1928) Über die isolierung verschiedener natürlicher Phosphorsäure-verbindungen und die Frage ihrer Einheitlichkeit. Biochem Z 194:306-327

Lohmann K (1934) Über die enzymatische Aufspaltung der Kreatinphosporsäure, zugleich en Beitrag zur Muskelkontraktion. Biochem Z 271:264-277

Lundsgaard E (1930a) Untersuchungen über Muskelkontraktionen ohne Milchsäurebildung. Biochem Z 217:162-177

Lundsgaard E (1930b) Weitere Untersuchungen über Muskelkontraktionen ohne Milchsäurebildung. Biochem Z 227:51-82

Mader A (2003) Glycolysis and oxidative phosphorylation as a function of cytosolic phosphorylation state and power output of the muscle cell. Eur J Appl Physiol 88:317-338. https://doi.org/ 10.1007/s00421-002-0676-3

Magder SA, Daughters GT, Hung J, Alderman EL, Ingels NB Jr (1987) Adaptation of human left ventricular volumes to the onset of supine exercise. Eur J Appl Physiol 56:467-473. https://doi.org/10.1007/BF00417777

Mahler M (1985) First-order kinetics of muscle oxygen consumption, and equivalent proportionality between $\dot{V} O_{2}$ and phophorylcreatine level. Implications for the control of respiration. J Gen Physiol 86:135-165. https://doi.org/10.1085/jgp.86.1.135

Malliani A, Pagani M, Lombardi F, Cerutti S (1991) Cardiovascular neural regulation explored in the frequency domain. Circulation 84:482-492. https://doi.org/10.1161/01.cir.84.2.482

Marconi C, Heisler N, Meyer M, Weitz H, Pendergast DR, Cerretelli P, Piiper J (1988) Blood flow distribution and its temporal variability in stimulated dog gastrocnemius muscle. Respir Physiol 74:1-13. https://doi.org/10.1016/0034-5687(88)90135-1

Marey EJ (1863) Physiologie médicale de la circulation du sang. Delahaye, Paris

Margaria R (1963) A mathematical treatment of the blood dissociation curve for oxygen. Clin Chem 9:745-762. https://doi.org/10.1093/ clinchem/9.6.745

Margaria R (1968) Capacity and power of the energy processes in muscle activity: their practical relevance in athletics. Int $\mathrm{Z}$ Angew Physiol 25:352-360. https://doi.org/10.1007/BF00699625

Margaria R, Edwards HT, Dill DB (1933) The possible mechanism of contracting and paying the oxygen debt and the role of lactic acid in muscular contraction. Am J Physiol 106:689-714. https://doi. org/10.1152/ajplegacy.1933.106.3.689

Margaria R, Cerretelli P, di Prampero PE, Massari C, Torelli G (1963) Kinetics and mechanism of oxygen debt contraction in man. J Appl Physiol 18:371-377. https://doi.org/10.1152/jappl.1963. 18.2.371

Margaria R, Cerretelli P, Mangili F (1964) Balance and kinetics of anaerobic energy release during strenuous exercise in man. $\mathbf{J}$ Appl Physiol 19:623-628. https://doi.org/10.1152/jappl.1964. 19.4.623

Margaria R, di Prampero PE, Aghemo P, Derevenco P, Mariani M (1971) Effect of a steady state exercise on maximal anaerobic power in man. J Appl Physiol 30:885-889. https://doi.org/10. 1152/jappl.1971.30.6.885

Mayer JR (1845) Die organische Bewegung in ihrem Zusammenhang mit der Stoffwechsel. Dreholerchen, Heilbronn

Mayow J (1681) Opera Omnia Medico-Physica Tractatibus Quinque Comprehensa. Elsevier, Den Haag 
McCloskey DI, Mitchell JH (1972) Reflex cardiovascular and respiratory responses originating in exercising muscle. J Physiol 224:173-186. https://doi.org/10.1113/jphysiol.1972.sp009887

McIlveen SA, Hayes SG, Kaufman MP (2001) Both central command and exercise pressor reflex reset carotid sinus baroreflex. Am J Physiol Heart Circ Physiol 280:H1454-H1463. https://doi.org/ 10.1152/ajpheart.2001.280.4.H1454

Meyer RA (1988) A linear model of muscle respiration explains monoexponential phosphocreatine changes. Am J Physiol Cell Physiol 254:C548-C553. https://doi.org/10.1152/ajpcell.1988. 254.4.C548

Meyer RA, Sweeney HL, Kushmerick MJ (1984) A simple analysis of the "phosphocreatine shuttle." Am J Physiol Cell Physiol 246:C365-C377. https://doi.org/10.1152/ajpcell.1984.246.5. C365

Meyer M, Rahmel A, Marconi C, Grassi B, Cerretelli P, Cabrol C (1994) Adjustment of cardiac output to step exercise in heart transplant recipients. Z Kardiol 83(suppl 3):103-109

Meyerhof O (1920) Die Energieumwandlungen im Muskel. I. Über die Beziehungen der Milchsäure zur Wärmebildung und Arbeitsleistung des Muskels in der Anaerobiose. Pflüg Arch 182:232-283. https://doi.org/10.1007/BF01723747

Meyerhof O (1921) Die Energieumwandlungen im Muskel. V. Milchsäurbildung Und Mechanische Arbeit. Pflüg Arch 191:128-183. https://doi.org/10.1007/BF01722479

Meyerhof O (1922) Die Energieumwandlungen im Muskel. VI. Über den Ursprung der Kontraktionswärme. Pflüg Arch 195:22-74. https://doi.org/10.1007/BF01723268

Meyerhof O (1924) Die Energieumwandlungen im Muskel. VII. Weitere Unterschinen über den Ursprung der Kontraktionswärme. Pflüg Arch 204:295-331. https://doi.org/10.1007/BF01731201

Michelini LC, O'Leary DS, Raven PB, Nóbrega AC (2015) Neural control of circulation and exercise: a translational approach disclosing interactions between central command, arterial baroreflex, and muscle metaboreflex. Am J Physiol Heart Circ Physiol 309:H381-H389. https://doi.org/10.1152/ajpheart.00077.2015

Mitchell PD (1979) The ninth Sir Hans Krebs Lecture. Compartmentation and communication in living systems. Ligand conduction: a general catalytic principle in chemical, osmotic and chemiosmotic reaction systems. Eur J Biochem 95:1-20. https://doi.org/ 10.1111/j.1432-1033.1979.tb12934.x

Mitchell PD (2004) Foundations of vectorial metabolism and osmochemistry. Biosci Rep 24:386-434. https://doi.org/10.1007/ s10540-005-2739-2

Mitchell PD (2011) Chemiosmotic coupling in oxidative and photosynthetic phosphorylation. Biochim Biophys Acta 1807:1507-1538. https://doi.org/10.1016/j.bbabio.2011.09.018

Monod H, Scherrer J (1965) The work capacity of a synergic muscular group. Ergonomics 8:329-338. https://doi.org/10.1080/00140 136508930810

Mortensen SP, Saltin B (2014) Regulation of the skeletal muscle blood flow in humans. Exp Physiol 99:1552-1558. https://doi.org/10. 1113/expphysiol.2014.081620

Mortensen SP, Thaning P, Nyberg M, Saltin B, Hellsten Y (2011) Local release of ATP into the arterial inflow and venous drainage of human skeletal muscle: insight from ATP determination with the intravascular microdialysis technique. J Physiol 589:1847-1857. https://doi.org/10.1113/jphysiol.2010.203034

Morton RH (1996) A 3-parameter critical power model. Ergonomics 39:611-619. https://doi.org/10.1080/00140139608964484

Murthy SE, Dubin AE, Patapoutian A (2017) Piezos thrive under pressure: mechanically activated ion channels in health and disease. Nat Rev Mol Cell Biol 18:771-783. https://doi.org/10.1038/nrm. 2017.92

Muth CM, Radermacher P, Pittner A, Steinacker J, Schabana R, Hamich S Paulat K, Calzia E (2003) Arterial blood gases during diving in elite apnea divers. Int J Sports Med 24:104-107. https:// doi.org/10.1055/s-2003-38401

Myers D, Imai K, Yonetani D (1990) Comparative least-squares analysis of hemoglobin oxygen equilibrium curves. Biophys Chem 37:323-340. https://doi.org/10.1016/0301-4622(90)88032-n

Nachmanson D (1928) Über den Zerfall der Kreatinphosphorsäure in Zusammenhang mit del Tätigkeit des Muskels. Biochem Z 196:73-97

Nadel ER, Cafarelli E, Roberts MF, Wenger CB (1979) Circulatory regulation during exercise in different ambient temperatures. J Appl Physiol 46:430-437. https://doi.org/10.1152/jappl.1979. 46.3.430

Naeije R, Badagliacca R (2017) The overloaded right heart and ventricular interdependence. Cardiovasc Res 113:1474-1485. https:// doi.org/10.1093/cvr/cvx160

Nattie E, Li A (2012) Central chemoreceptors: locations and functions. Compr Physiol 2:221-254. https://doi.org/10.1002/cphy.c100083

Needham DM (1971) Machina Carnis. Cambridge University Press, Cambridge

Nielsen HB (2003) Arterial desaturation during exercise in man: implication for $\mathrm{O}_{2}$ uptake and work capacity. Scand J Med Sci Sports 13:339-358. https://doi.org/10.1046/j.1600-0838.2003.00325.x

Nóbrega AC, Williamson JW, Garcia JA, Mitchell JH (1997) Mechanisms for increasing stroke volume during static exercise with fixed heart rate in humans. J Appl Physiol 83:712-717. https:// doi.org/10.1152/jappl.1997.83.3.712

Norton KH, Boushel R, Strange S, Saltin B, Raven PB (1999a) Resetting of the carotid arterial baroreflex during dynamic exercise in humans. J Appl Physiol 87:332-338. https://doi.org/10.1152/ jappl.1999.87.1.332

Norton KH, Gallagher KM, Smith SA, Querry RG, Welch-O'Connor RM, Raven PB (1999b) Carotid baroreflex function during prolonged exercise. J Appl Physiol 87:339-347. https://doi.org/10. 1152/jappl.1999.87.1.339

Ogoh S, Fisher JP, Fadel PJ, Raven PB (2007) Increases in central blood volume modulate carotid baroreflex resetting during dynamic exercise in humans. J Physiol 581:405-418. https:// doi.org/10.1113/jphysiol.2006.125112

O'Leary DD, Kimmerly DS, Cechetto AD, Shoemaker JK (2003) Differential effect of head-up tilt on cardiovagal and sympathetic baroreflex sensitivity in humans. Exp Physiol 88:769-774. https://doi.org/10.1113/eph8802632

O’Riordan JF, Goldstick TK, Vida LN, Honig GR, Ernest JT (1985) Modelling whole blood oxygen equilibrium: comparison of nine different models fitted to normal human data. Adv Exp Med Biol 191:505-522. https://doi.org/10.1007/978-1-4684-3291-6_51

Otis AB (1964) Quantitative relationships in steady-state gas exchange. In: Fenn WO, Rahn H (eds) Handbook of physiology, respiration, section 3, vol 1. American Physiological Society, Washington, pp 681-698

Otis AB, Rahn H, Fenn WO (1948) Alveolar gas changes during breath holding. Am J Physiol 152:674-686. https://doi.org/10.1152/ ajplegacy.1948.152.3.674

Palada I, Bakovic D, Valic Z, Obad A, Ivancev V, Eterovic D, Shoemaker JK, Dujic Z (2008) Restoration of hemodynamics in apnea struggle phase in association with involuntary breathing movements. Respir Physiol Neurobiol 161:174-181. https://doi.org/ 10.1016/j.resp.2008.01.008

Papelier Y, Escourrou P, Gauthier JP, Rowell LB (1994) Carotid baroreflex control of blood pressure and heart rate in men during dynamic exercise. J Appl Physiol 77:502-506. https://doi. org/10.1152/jappl.1994.77.2.502

Pappenheimer JR (1950) Standardization of definitions and symbols in respiratory physiology. Fed Proc 9:602-605 
Patel AJ, Honoré E (2001) Molecular physiology of oxygen-sensitive potassium channels. Eur Respir J 18:221-227. https://doi.org/10. 1183/09031936.01.00204001

Paterson DH, Whipp BJ (1991) Asymmetries of $\mathrm{O}_{2}$ uptake transients at the on- and offset of heavy exercise in humans. J Physiol 443:575-586. https://doi.org/10.1113/jphysiol.1991.sp018852

Pauling L (1935) The oxygen equilibrium of hemoglobin and its structural interpretation. Proc Natl Acad Sci 21:186-191. https://doi. org/10.1073/pnas.21.4.186

Pendergast DR, Lundgren CEG (2009) The underwater environment: cardiopulmonary, thermal and energetic demand. J Appl Physiol 106:276-283. https://doi.org/10.1152/japplphysiol.90984.2008

Périard JD, Eijsvogel TMH, Daanen HAM (2021) Exercise under heat stress: thermoregulation, hydration, performance implications, and mitigation strategies. Physiol Rev 101:1873-1979. https:// doi.org/10.1152/physrev.00038.2020

Perini R, Veicsteinas A (2003) Heart rate variability and autonomic activity at rest and during exercise in various physiological conditions. Eur J Appl Physiol 90:317-325. https://doi.org/10.1007/ s00421-003-0953-9

Perini R, Tironi A, Gheza A, Butti F, Moia C, Ferretti G (2008) Heart rate and blood pressure time courses during prolonged dry apnoea in breath-hold divers. Eur J Appl Physiol 104:1-7. https:// doi.org/10.1007/s00421-008-0771-1

Perini R, Gheza A, Moia C, Sponsiello N, Ferretti G (2010) Cardiovascular time courses during prolonged immersed static apnoea. Eur J Appl Physiol 110:277-283. https://doi.org/10.1007/ s00421-010-1489-4

Perkins JF (1964) Historical development of respiratory physiology. In: Fenn WO, Rahn H (eds) Handbook of physiology, respiration, vol 1. American Physiological Society, Washington, pp 1-58

Perutz MF (1970) Stereochemistry of cooperative effects in haemoglobin. Nature 228:726-734. https://doi.org/10.1038/228726a0

Pettenkofer M, Voigt C (1866) Untersuchungen über den Stoffverbrauch des normalen Menschen. Biochem Z 2:459-573

Pickering TG, Gribbin B, Petersen ES, Cunningham DJ, Sleight P (1971) Comparison of the effects of exercise and posture on the baroreflex in man. Cardiovasc Res 5:582-586. https://doi.org/ $10.1093 / \mathrm{cvr} / 5.4 .582$

Pickering TG, Gribbin B, Petersen ES, Cunningham DJ, Sleight P (1972) Effects of autonomic blockade on the baroreflex in man at rest and during exercise. Circ Res 30:177-185. https://doi.org/ 10.1161/01.res.30.2.177

Piiper J (1992) Diffusion-perfusion inhomogeneity and alveolar-arterial $\mathrm{O}_{2}$ diffusion limitation: theory. Respir Physiol 87:349-356. https://doi.org/10.1016/0034-5687(92)90016-p

Piiper J (2000) Perfusion, diffusion and their heterogeneities limiting blood-tissue $\mathrm{O}_{2}$ transfer in muscle. Acta Physiol Scand 168:603607. https://doi.org/10.1046/j.1365-201x.2000.00711.x

Piiper J, Scheid P (1981) Model for capillary-alveolar equilibration with special reference to $\mathrm{O}_{2}$ uptake in hypoxia. Respir Physiol 46:193-208. https://doi.org/10.1016/0034-5687(81)90121-3

Piiper J, Scheid P (1999) Modeling oxygen availability to exercising muscle. Respir Physiol 118:95-101. https://doi.org/10.1016/ s0034-5687(99)00082-1

Piiper J, di Prampero PE, Cerretelli P (1968) Oxygen debt and highenergy phosphates in gastrocnemius muscle of the dog. Am J Physiol 215:523-531. https://doi.org/10.1152/ajplegacy.1968. 215.3.523

Piiper J, Meyer M, Scheid P (1984) Dual role of diffusion in tissue gas exchange: blood-tissue equilibration and diffusion shunt. Respir Physiol 56:131-144. https://doi.org/10.1016/0034-5687(84) 90099-9
Piiper J, Pendergast DR, Marconi C, Meyer M, Heisler N, Cerretelli $\mathrm{P}$ (1985) Blood flow distribution in dog gastrocnemius muscle at rest and during stimulation. J Appl Physiol 58:2068-2074. https://doi.org/10.1152/jappl.1985.58.6.2068

Poole DC, Jones AM (2012) Oxygen uptake kinetics. Compr Physiol 2:1-64. https://doi.org/10.1002/cphy.c100072

Poole DC, Ward SA, Gardner GW, Whipp BJ (1988) Metabolic and respiratory profile of the upper limit for prolonged exercise in man. Ergonomics 31:1265-1279. https://doi.org/10.1080/00140 138808966766

Poole DC, Barstow TJ, Gaesser GA, Willis WT, Whipp BJ (1994) $\dot{V} O_{2}$ slow component: physiological and functional significance. Med Sci Sports Exerc 26:1354-1358

Poole DC, White M, Whipp BJ (2015) The discovery of oxygen. Hektoen International 7: ISSN 2155-3017

Poole DC, Burnley M, Vanhatalo A, Rossiter HB, Jones AM (2016) Critical power: an important fatigue threshold in exercise physiology. Med Sci Sports Exerc 48:2320-2334. https://doi.org/10. 1249/MSS.0000000000000939

Poole DC, Rossiter HB, Brooks GA, Gladden LB (2021) The anaerobic threshold: 50 + years of controversy. J Physiol 599:737-767. https://doi.org/10.1113/JP279963

Poole DC, Musch TI, Colburn TD (2022) Oxygen flux from capillary to mitochondria: integration of contemporary discoveries. Eur J Appl Physiol. 122:7-28. https://doi.org/10.1007/ s00421-021-04854-7

Potts JT, Shi X, Raven PB (1993) Carotid baroreflex responsiveness during dynamic exercise in humans. Am J Physiol Heart Circ Physiol 265:H1928-H1938. https://doi.org/10.1152/ajpheart. 1993.265.6.H1928

Prefaut C, Durand F, Mucci P, Caillaud C (2000) Exercise-induced arterial hypoxaemia in athletes: a review. Sports Med 30:47-61. https://doi.org/10.2165/00007256-200030010-00005

Premont RT, Reynolds JD, Zhang R, Stamler JS (2020) Role of nitric oxide carried by hemoglobin in cardiovascular physiology: developments on a three-gas respiratory cycle. Circ Res 126:129-158. https://doi.org/10.1161/CIRCRESAHA.119.315626

Prisk GK, Elliott AR, Guy HJ, Kosonen JM, West JB (1995) Pulmonary gas exchange and its determinants during sustained microgravity on Spacelabs SLS-1 and SLS-2. J Appl Physiol 79:12901298. https://doi.org/10.1152/jappl.1995.79.4.1290

Racker E (1983) Resolution and reconstitution of biological pathways from 1919 to 1984. Fed Proc 42:2899-2909

Rahn H, Fenn WO (1955) A graphical analysis of the respiratory gas exchange. American Physiological Society, Washington

Rahn H, Otis AB (1949) Continuous analysis of alveolar gas composition during work, hyperpnea, hypercapnia and anoxia. $\mathbf{J}$ Appl Physiol 1:717-724. https://doi.org/10.1152/jappl.1949.1. 10.717

Ranvier L (1873) Propriété et structure différente des muscles rouges et des muscles blancs chez les lapins et chez les raies. CR Acad Sci 77:1030-1034

Raven PB, Fadel PJ, Ogoh S (2006) Arterial baroreflex resetting during exercise: a current perspective. Exp Physiol 91:37-49. https:// doi.org/10.1113/expphysiol.2005.032250

Raven PB, Young BE, Fadel PJ (2019) Arterial baroreflex resetting during exercise in humans: underlying signalling mechanisms. Exerc Sport Sci Rev 47:129-141. https://doi.org/10.1249/JES. 0000000000000190

Reaburn PRJ, McKinnon LT (1990) Blood lactate responses in older swimmers during active and passive recovery following maximal sprint swimming. Eur J Appl Physiol 61:246-250. https://doi.org/ 10.1007/BF00357607 
Rennie DW, di Prampero PE, Cerretelli P (1971) Effects of water immersion on cardiac output, heart rate and stroke volume of man at rest and during exercise. Med Sport 24:223-228

Retailleau K, Duprat F, Arhatte M, Ranade SS, Peyronnet R, Martins JR, Jodar M, Moro C, Offermanns S, Feng Y, Demolombe S, Patel A, Honoré E (2015) Piezo 1 in smooth muscle cells is involved in hypertension-dependent arterial remodeling. Cell Rep 13:1161-1171. https://doi.org/10.1016/j.celrep.2015.09.072

Ribeiro JP, Hughes V, Fielding RA, Holden W, Evans W, Knuttgen HG (1986) Metabolic and ventilatory responses to steady state exercise relative to lactate thresholds. Eur J Appl Physiol 55:215221. https://doi.org/10.1007/BF00715008

Richardson RS, Wary C, Wray DW, Hoff J, Rossiter HB, Layec G, Carlier PG (2015) MRS evidence of adequate $\mathrm{O}_{2}$ supply in human skeletal muscle at the onset of exercise. Med Sci Sports Exerc 47:2299-2307. https://doi.org/10.1249/MSS.0000000000000675

Roach RC, Koskolou MD, Calbet JA, Saltin B (1999) Arterial O ${ }_{2}$ content and tension in regulation of cardiac output and leg blood flow during exercise in humans. Am J Physiol Heart Circ Physiol 276:H438-H445. https://doi.org/10.1152/ajpheart.1999.276.2. $\mathrm{H} 438$

Robinson BF, Epstein SE, Beiser GD, Braunwald E (1966) Control of heart rate by the autonomic nervous system: studies in man on the interrelation between baroreceptor mechanisms and exercise. Circ Res 19:400-411. https://doi.org/10.1161/01.res.19.2.400

Roca J, Hogan MC, Story D, Bebout DE, Haab P, Gonzalez R, Ueno O, Wagner PD (1989) Evidence for tissue diffusion limitation of in normal humans. J Appl Physiol 67:291-299. https://doi.org/ 10.1152/jappl.1992.73.3.1067

Rossiter HB, Ward SA, Doyle VL, Howe FA, Griffiths JA, Whipp BJ (1999) Inferences from pulmonary $\mathrm{O}_{2}$ uptake with respect to intramuscular [phosphocreatine] kinetics during moderate exercise in humans. J Physiol 518:921-932. https://doi.org/10.1111/j. 1469-7793.1999.0921p.x

Rossiter HB, Ward SA, Kowalchuk JM, Howe FA, Griffiths JR, Whipp BJ (2002) Dynamic asymmetry of phosphocreatine concentration and $\mathrm{O}_{2}$ uptake between the on- and off-transients of moderateand high-intensity exercise in humans. J Physiol 541:991-1002. https://doi.org/10.1113/jphysiol.2001.012910

Rossiter HB, Howe FA, Ward SA (2005) Intramuscular phosphate and pulmonary $\mathrm{V}_{2}$ kinetics during exercise: implications for control of skeletal muscle $\mathrm{O}_{2}$ consumption. In: Jones AM, Poole DC (eds) Oxygen uptake kinetics in sport, exercise and medicine. Routledge, London, pp 154-184

Rowell LB (1974) Human cardiovascular adjustments to exercise and thermal stress. Physiol Rev 54:75-159. https://doi.org/10.1152/ physrev.1974.54.1.75

Rowell LB, Brengelmann GL, Blackmon JR, Twiss RD, Kusumi F (1968) Disparities between aortic and peripheral pulse pressures induced by upright exercise and vasomotor changes in man. Circulation 37:954-964. https://doi.org/10.1161/01.cir.37.6.954

Rowell LB, O'Leary DS, Kellogg DL Jr (1996) Integration of cardiovascular control systems in dynamic exercise. Handbook of physiology. Exercise: regulation and integration of multiple systems, section 12, chapter 17. American Physiological Society, Bethesda, pp 770-838

Roy TK, Secomb TW (2019) Effects of pulmonary flow heterogeneity on oxygen transport parameters in exercise. Respir Physiol Neurobiol 261:75-79. https://doi.org/10.1016/j.resp.2018.10.004

Rubner M (1894) Die Quelle der tierischen Wärme. Z Biol 30:73-142

Saltin B, Blomqvist G, Mitchell JH, Johnson RL Jr, Wildenthal K, Chapman CB (1968) Response to exercise after bed rest and after training. Circulation 38(suppl 5):1-78

Saltin B, Rådegran G, Koskolou MD, Roach RC (1998) Skeletal muscle blood flow in humans and its regulation during exercise. Acta
Physiol Scand 162:421-436. https://doi.org/10.1046/j.1365201X.1998.0293e.x

Schneider SM, Watenpaugh DE, Lee SMC, Ertl AC, Williams WJ, Ballard RE, Hargens AR (2002) Lower-body negative-pressure exercise and bed-rest-mediated orthostatic intolerance. Med Sci Sports Exerc 34:1446-1453. https://doi.org/10.1097/00005768200209000-00008

Schrage WG, Dietz NM, Joyner MJ (2006) Effects of combined inhibition of ATP-sensitive potassium channels, nitric oxide, and prostaglandins on hyperemia during moderate exercise. J Appl Physiol 100:1506-1512. https://doi.org/10.1152/japplphysiol. 01639.2005

Seals DR, Victor RG (1991) Regulation of muscle sympathetic nerve activity during exercise in humans. Exerc Sports Sci Rev 19:313349. https://doi.org/10.1097/00005768-200209000-00008

Secher NH, Clausen JP, Klausen K, Noer I, Trap-Jensen J (1977) Central and regional circulatory effects of adding arm exercise to leg exercise. Acta Physiol Scand 100:288-297. https://doi.org/10. 1111/j.1748-1716.1977.tb05952.x

Séguin A, Lavoisier A (1789) Premier mémoire sur la respiration des animaux. Mem Acad R Sci 566-584

Severinghaus JW (1979) Simple, accurate expressions for human blood oxygen dissociation computations. J Appl Physiol 46:599-602. https://doi.org/10.1152/jappl.1979.46.3.599

Sheldahl LM, Tristani FE, Clifford PS, Hughes CV, Sobocinski KA, Morris RD (1987) Effect of head-out water immersion on cardiorespiratory response to dynamic exercise. J Am Coll Cardiol 10:1254-1258. https://doi.org/10.1016/s0735-1097(87)80127-4

Singel DJ, Stamler JS (2005) Chemical physiology of blood flow regulation by red blood cells: the role of nitric oxide and S-nitrosohemoglobin. Annu Rev Physiol 67:99-145. https://doi.org/10.1016/ s0735-1097(87)80127-4

Sivieri A, Fagoni N, Bringard A, Capogrosso M, Perini R, Ferretti G (2015) A beat-by-beat analysis of cardiovascular responses to dry resting and exercise apnoeas in elite divers. Eur J Appl Physiol 115:119-128. https://doi.org/10.1007/s00421-014-2992-9

Song N, Guan R, Jiang Q, Hassanzadeh CJ, Chu Y, Zhao X, Wang X, Yang D, Du Q, Chu XP, Shen L (2016) Acid-sensing ion channels are expressed in the ventrolateral medulla and contribute to central chemoreception. Sci Rep 6:38777. https://doi.org/10. 1038/srep38777

Springer C, Barstow TJ, Wasserman K, Cooper DM (1991) O 2 uptake and heart rate responses during hypoxic exercise in children and adults. Med Sci Sports Exerc 23:71-79

Spyer MK, Gourine AV (2009) Chemosensory pathways in the brainstem controlling cardiorespiratory activity. Philos Trans R Soc Lond B 364:2603-2610. https://doi.org/10.1098/rstb.2009.0082

Stamler JS, Jia L, Eu JP, McMahon TJ, Demchenko IT, Bonaventura J, Gernert K, Piantadosi CA (1997) Blood flow regulation by $\mathrm{S}$-nitrosohemoglobin in the physiological oxygen gradient. Science 276:2034-2037. https://doi.org/10.1126/science.276.5321. 2034

Steinacker JM, Liu Y, Böning D, Halder A, Maassen N, Thomas A, Stauch M (1996) Lung diffusion capacity, oxygen uptake, cardiac output and oxygen transport during exercise before and after an Himalayan expedition. Eur J Appl Physiol 74:187-193. https:// doi.org/10.1007/BF00376512

Steinback CD, O'Leary DD, Bakker J, Cechetto AD, Ladak HM, Shoemaker JK (2005) Carotid distensibility, baroreflex sensitivity, and orthostatic stress. J Appl Physiol 99:64-70. https://doi.org/10. 1152/japplphysiol.01248.2004

Stella G (1928) The concentration and diffusion of inorganic phosphate in living muscle. J Physiol Lond 66:19-31. https://doi.org/10. 1113/jphysiol.1928.sp002504 
Stenberg J, Ekblom B, Messin R (1966) Hemodynamic response to work at simulated altitude, 4,000 m. J Appl Physiol 21:15891594. https://doi.org/10.1152/jappl.1966.21.5.1589

Stenberg J, Åstrand PO, Ekblom B, Royce J, Saltin B (1967) Hemodynamic response to work with different muscle groups, sitting and supine. J Appl Physiol 22:61-70. https://doi.org/10.1152/ jappl.1967.22.1.61

Stocker SD, Sved AF, Andresen MC (2019) Missing pieces of the Piezo1/Piezo2 baroreceptor hypothesis: an autonomic perspective. J Neurophysiol 122:1207-1212. https://doi.org/10.1152/jn. 00315.2019

Sundblad P, Haruna Y, Tedner B, Linnarsson D (2000) Short-term cardiovascular responses to rapid whole-body tilting during exercise. Eur J Appl Physiol 81:259-270. https://doi.org/10.1007/ s004210050041

Sundblad P, Spaak J, Kaijser L (2014) Time courses of central hemodynamics during rapid changes in posture. J Appl Physiol 116:1182-1188. https://doi.org/10.1152/japplphysiol.00690. 2013

Sutton JR, Reeves JT, Wagner PD, Groves BM, Cymerman A, Malconian MK, Rock PB, Young PM, Walter SD, Houston CS (1988) Operation Everest II: oxygen transport during exercise at extreme simulated altitude. J Appl Physiol 64:1309-1321. https://doi.org/ 10.1152/jappl.1988.64.4.1309

Taboni A, Vinetti G, Bruseghini P, Camelio S, D'Elia M, Moia C, Ferretti G, Fagoni N (2018) Cardiovascular responses to dry apnoeas at exercise in air and in pure oxygen. Respir Physiol Neurobiol 255:17-21. https://doi.org/10.1016/j.resp.2018.05.003

Taboni A, Fagoni N, Moia C, Vinetti G, Ferretti G (2019) Gas exchange and cardiovascular responses during breath-holding in divers. Respir Physiol Neurobiol 267:27-34. https://doi.org/10.1016/j. resp.2019.06.002

Taboni A, Fagoni N, Fontolliet T, Grasso GS, Moia C, Vinetti G, Ferretti G (2020) Breath holding as an example of extreme hypoventilation: experimental testing of a new model describing alveolar gas pathways. Exp Physiol 105:2216-2225. https://doi.org/10. 1113/EP088977

Taboni A, Fagoni N, Fontolliet T, Moia C, Vinetti G, Ferretti G (2021a) A closed-loop approach to the study of the baroreflex dynamics during posture changes at rest and at exercise in humans. Am J Physiol Regul Integr Comp Physiol. https://doi.org/10.1152/ ajpregu.00167.2021

Taboni A, Vinetti G, Fontolliet T, Grasso GS, Tam E, Moia C, Ferretti G, Fagoni N (2021b) Baroreflex responses during dry resting and exercise apnoeas in air and pure oxygen. Eur J Appl Physiol 121:539-547. https://doi.org/10.1007/s00421-020-04544-w

Tam E, Rossi H, Moia C, Berardelli C, Rosa G, Capelli C, Ferretti G (2012) Energetics of running in top-level marathon runners from Kenya. Eur J Appl Physiol 112:3797-3806. https://doi. org/10.1007/s00421-012-2357-1

Tanabe N, Todoran TM, Zenk GM, Bunton BR, Wagner WW Jr, Presson RG Jr (1998) Perfusion heterogeneity in the pulmonary acinus. J Appl Physiol 84:933-938. https://doi.org/10. 1152/jappl.1998.84.3.933

Taylor CR, Heglund NC (1982) Energetics and mechanics of terrestrial locomotion. Annu Rev Physiol 44:97-107. https://doi. org/10.1146/annurev.ph.44.030182.000525

Taylor CR, Weibel ER (1981) Design of the mammalian respiratory system. I. Problem and strategy. Respir Physiol 44:1-10. https://doi.org/10.1016/0034-5687(81)90073-6

Thomsen JJ, Rentsch RL, Robach P, Calbet JAL, Boushel R, Rasmussen P, Juel C, Lundby C (2007) Prolonged administration of recombinant human erythropoietin increases submaximal performance more than maximal aerobic capacity. Eur J Appl Physiol 101:481-486. https://doi.org/10.1007/ s00421-007-0522-8
Tien YK, Gabel RA (1977) Prediction of $\mathrm{PO}_{2}$ from $\mathrm{SO}_{2}$ using the standard oxygen hemoglobin equilibrium curve. J Appl Physiol 42:985-987. https://doi.org/10.1152/jappl.1977.42.6.985

Tocco F, Crisafulli A, Melis F, Porru C, Pittau G, Milia R, Toncu A (2012) Cardiovascular adjustments in breath-hold diving: comparison between divers and non-divers in simulated dynamic apnoea. Eur J Appl Physiol 112:543-554. https://doi.org/10. 1007/s00421-011-2006-0

Tocco F, Marongiu E, Pinna M, Roberto S, Pusceddu M, Angius L, Migliaccio G, Milia R, Concu A, Crisafulli A (2013) Assessment of circulatory adjustments during underwater apnoea in elite divers by means of a portable device. Acta Physiol 207:290-298. https://doi.org/10.1111/apha.12000

Tsuchimochi H, Hayes SG, McCord JL, Kaufman MP (2009) Both central command and exercise pressor reflex activate cardiac sympathetic nerve activity in decerebrate cats. Am J Physiol Heart Circ Physiol 296:H1157-H1163. https://doi.org/10.1152/ ajpheart.01219.2008

Turner DL (1991) Cardiovascular and respiratory control mechanisms during exercise: an integrated view. J Exp Biol 160:309340. https://doi.org/10.1242/JEB.160.1.309

Vallais F, Baselli G, Lucini D, Pagani M, Porta A (2009) Spontaneous baroreflex sensitivity estimates during graded bicycle exercise: a comparative study. Physiol Meas 30:201-213. https:// doi.org/10.1088/0967-3334/30/2/007

Vinetti G, Taboni A, Bruseghini P, Camelio S, D’Elia M, Fagoni N, Moia C, Ferretti G (2019) Experimental validation of the 3 -parameter critical power model in cycling. Eur J Appl Physiol 119:941-949. https://doi.org/10.1007/s00421-019-04083-z

Vogel JA, Gleser MA (1972) Effect of carbon monoxide on oxygen transport during exercise. J Appl Physiol 32:234-239. https:// doi.org/10.1152/jappl.1972.32.2.234

von Staden H (1989) The art of medicine in early Alexandria. Cambridge University Press, Cambridge

Wagner PD (1977) Diffusion and chemical reaction in pulmonary gas exchange. Physiol Rev 57:257-312. https://doi.org/10.1152/ physrev.1977.57.2.257

Wagner PD (1992) Ventilation-perfusion matching during exercise. Chest 101:192S-198S. https://doi.org/10.1378/chest.101.5_ supplement.192s

Wagner PD (2015) The physiological basis of pulmonary gas exchange: implications for clinical interpretation of arterial blood gases. Eur Respir J 45:227-243. https://doi.org/10.1183/ 09031936.00039214

Wagner PD, West JB (1972) Effects of diffusion impairment on $\mathrm{O}_{2}$, and $\mathrm{CO}_{2}$ time courses in pulmonary capillaries. J Appl Physiol 33:62-71. https://doi.org/10.1152/jappl.1972.33.1.62

Walsh B, Tonkonogi M, Söderlund K, Hultman E, Saks V, Sahlin K (2001) The role of phosphorylcreatine and creatine in the regulation of mitochondrial respiration in human skeletal muscle. J Physiol 537:971-978. https://doi.org/10.1111/j.1469-7793. 2001.00971.x

Wang SP, Chennupathi R, Kaur H, Iring A, Wettschureck N, Offermanns $S$ (2016) Endothelial cation channel PIEZO 1 controls blood pressure by mediating flow-induced ATP release. J Clin Invest 126:4527-4536. https://doi.org/10.1172/JCI87343

Wasserman K, Whipp BJ, Castagna J (1974) Cardiodynamic hyperpnea: hyperpnea secondary to cardiac output increase. J Appl Physiol 36:457-464. https://doi.org/10.1152/jappl.1974.36.4. 457

Weibel ER (1972) Morphometric estimation of pulmonary diffusion capacity. V. Comparative morphometry of alveolar lungs. Respir Physiol 14:26-43. https://doi.org/10.1016/00345687(72)90015-1 
Weibel ER (1999) Understanding the limitation of $\mathrm{O}_{2}$ supply through comparative physiology. Respir Physiol 118:85-93. https://doi. org/10.1016/s0034-5687(99)00084-5

Weissman C, Abraham B, Askanazi J, Milic-Emili J, Hyman AI, Kinney JM (1982) Effect of posture on the ventilatory response to $\mathrm{CO}_{2}$. J Appl Physiol 53:761-765. https://doi.org/10.1152/ jappl.1982.53.3.761

Whipp BJ (1994) Peripheral chemoreceptor control of exercise hyperpnea in humans. Med Sci Sports Exerc 26:337-347. https://doi.org/10.1249/00005768-199403000-00010

Whipp BJ, Mahler M (1980) Dynamics of pulmonary gas exchange during exercise. In: West JB (ed) Pulmonary gas exchange, vol II. Academic Press, New York, pp 33-96

Whipp BJ, Ward SA (1982) Cardiopulmonary coupling during exercise. J Exp Biol 100:175-193. https://doi.org/10.1242/ jeb.100.1.175

Whipp BJ, Ward SA (1990) Physiological determinants of pulmonary gas exchange kinetics during exercise. Med Sci Sports Exerc 22:62-71. https://doi.org/10.1249/00005768-19900 2000-00011

Whipp BJ, Wasserman K (1972) $\mathrm{O}_{2}$ uptake kinetics for various intensities of constant-load work. J Appl Physiol 33:351-356. https://doi.org/10.1152/jappl.1972.33.3.351

Whipp BJ, Rossiter HB, Ward SA, Avery D, Doyle VL, Howe FA, Griffiths JR (1999) Simultaneous determination of muscle ${ }^{31} \mathrm{P}$ and $\mathrm{O}_{2}$ uptake kinetics during whole body NMR spectroscopy. J Appl Physiol 86:742-747. https://doi.org/10.1152/jappl.1999. 86.2.742

Wieling W, Harms MP, ten Harkel AD, van Lieshout JJ, Sprangers RL (1996) Circulatory response evoked by a $3 \mathrm{~s}$ bout of dynamic leg exercise in humans. J Physiol 494:601-611. https://doi.org/10.1113/jphysiol.1996.sp021518
Wilson RJA, Teppema LJ (2016) Interaction of central and peripheral respiratory chemoreflexes. Compr Physiol 6:1005-1041. https://doi.org/10.1002/cphy.c140040

Wislicenus J (1873) Über die optisch-aktive Milchsäuren der Fleischflüssigkeit, die Paramilchsäure. Ann Chem 167:302-346

Woodson RD, Wills RE, Lenfant C (1978) Effect of acute and established anemia on $\mathrm{O}_{2}$ transport at rest, submaximal and maximal work. J Appl Physiol 44:36-43. https://doi.org/10.1152/jappl. 1978.44.1.36

Yoshida T, Whipp BJ (1994) Dynamic asymmetries of cardiac output transients in response to muscular exercise in man. $\mathrm{J}$ Physiol 480:355-359. https://doi.org/10.1113/jphysiol.1994.sp020365

Zamparo P, Cortesi M, Gatta G (2020) The energy cost of swimming and its determinants. Eur J Appl Physiol 120:41-66. https:// doi.org/10.1007/s00421-019-04270-y

Zha XM (2013) Acid-sensing ion channels: trafficking and synaptic function. Mol Brain 6:1. https://doi.org/10.1186/ 1756-6606-6-1

Zuntz N (1901) Über die Bedeutung der verschiedenen Nährstoffe als Erzeuger der Muskelkraft. Pflüg Arch 83:557-571. https:// doi.org/10.1007/BF01746509

Zuntz N, Geppert J (1886) Uber die Natur der normalen Atemreize und den Ort ihrer Wirkung. Pflüg Arch 38:337-338. https://doi.org/ 10.1007/BF01654665

Publisher's Note Springer Nature remains neutral with regard to jurisdictional claims in published maps and institutional affiliations. 